JACQUELINE DE MIRANDA KIAN

Topology Optimization Method applied to Design Channels Considering Non-Newtonian Fluid Flow 
JACQUELINE DE MIRANDA KIAN

\section{Topology Optimization Method applied to Design Channels Considering Non-Newtonian Fluid Flow}

Submitted in partial fulfilment of the requirements for the degree of Master of Science in Mechanical Engineering 
JACQUELINE DE MIRANDA KIAN

\section{Topology Optimization Method applied to Design Channels Considering Non-Newtonian Fluid Flow}

Submitted in partial fulfilment of the requirements for the degree of Master of Science in Mechanical Engineering

Field of Study:

Control and Mechanical Automation Engineering (3152)

Supervised by:

Prof. Dr. Emílio Carlos Nelli Silva 
Este exemplar foi revisado e corrigido em relação à versão original, sob responsabilidade única do autor e com a anuência de seu orientador.

São Paulo, de de

Assinatura do autor:

Assinatura do orientador:

Catalogação-na-publicação

Kian, Jacqueline

Topology Optimization Method applied to Design Channels Considering Non-Newtonian Fluid Flow / J. Kian -- versão corr. -- São Paulo, 2017.

$77 \mathrm{p}$.

Dissertação (Mestrado) - Escola Politécnica da Universidade de São Paulo. Departamento de Engenharia Mecatrônica e de Sistemas Mecânicos.

1.Métodos Topológicos (Otimização) 2.Método dos Elementos Finitos 3.Dinâmica dos Fluidos (Simulação) 4.Hemodinâmica I.Universidade de São Paulo. Escola Politécnica. Departamento de Engenharia Mecatrônica e de Sistemas Mecânicos II.t. 


\section{ACKNOWLEDGEMENTS}

To my supervisor, Prof. Emilio Carlos Nelli Silva for the attention, guidance and patience during the execution of this work.

To my boyfriend Luís Fernando Nogueira de Sá for the support and love, and for always giving me motivation to go on.

To my parents Vilson and Marlene and my brother Guilherme, for believing in me and supporting my decisions.

To my lab colleagues and to professor Juan Romero for the useful suggestions which were valuable to the development of this work. 


\section{RESUMO}

O estudo de escoamento de fluidos não-Newtonianos apresenta-se relevante no campo de bioengenharia, em especial no projeto de dispositivos para condução de sangue, como bypass arterial. Melhorias na redução de dissipação de energia e no dano às células sanguíneas causados por fluxos artificiais podem ser obtidas através do uso de técnicas de simulação e otimização numéricas. Deste modo, este trabalho propõe o estudo do projeto de canais para escoamentos incompressíveis em regime permanente de fluidos não-Newtonianos através do Método de Otimização Topológica baseado no método de densidade. O escoamento é modelado com as equações de Navier-Stokes acopladas com a equação constitutiva de Carreau-Yasuda para a viscosidade dinâmica, para que sejam considerados os efeitos das propriedades não-Newtonianas do sangue. O Método de Otimização Topológica distribue regiões de sólido e fluido, dada uma restrição de volume, dentro de um domínio especificado de modo a obter uma geometria e configuração que minimize a dissipação de energia, tensão de cisalhamento e vorticidade, utilizando a pseudo-densidade do material como variável de projeto. Para aplicar este método a sistemas fluidos, um meio poroso fictício, baseado na equação de Darcy, é introduzido. O modelo de escoamento é implementado em sua forma discreta utilizando o Método de Elementos Finitos através da plataforma OpenSource FEniCS, aplicada para automatizar a solução dos modelos matemáticos baseados em equações diferenciais, e o problema de otimização é resolvido utilizando a biblioteca DOLFIN-adjoint e otimizador IPOpt. Topologias otimizadas de canais para fluxo de sangue, com foco em bypass arterial, são apresentadas para ilustrar o método proposto.

Palavras-chave: Otimização Topológica. Fluidos Não-Newtonianos. Escoamento de Sangue. Bypass Arterial. 


\section{ABSTRACT}

The study of non-Newtonian flow is presents itself as relevant in bioengineering field, specially for design of devices that conduct blood, as arterial bypass grafts. Improvements in reducing energy dissipation and blood cell damage caused by artificial flows can be achieved by using numerical simulation and optimization methods. Thus, the present work proposes the study of design channels for steady, incompressible non-Newtonian flow, by using Topology Optimization Method based on the density method. The fluid flow is modeled with the Navier-Stokes equations coupled with Carreau-Yasuda constitutive equation for the dynamic viscosity to take into account the effects of the non-Newtonian blood properties. The Topology Optimization Method distributes regions of solid and fluid, given a volume constraint, within a specified domain in order to obtain a geometry and layout that minimizes energy dissipation, shear stress and vorticity by using the material pseudo-density as design variable. To apply this method to fluidic systems design, a fictional porous media based on Darcy equation is introduced. The flow model is implemented in its discrete form by using the Finite Element Method through the OpenSource platform FEniCS, applied to automate the solution of mathematical models based on differential equations. The optimization problem is solved by using the library DOLFIN-adjoint and IPOpt optimizer. Optimized topologies of channels for blood flow, focusing in arterial bypass grafts, are presented to illustrate the proposed method.

Keywords: Topology Optimization. Non-Newtonian Fluids. Blood Flow. Arterial Bypass. 


\section{LIST OF FIGURES}

Figure 1.1 - Influence of red blood cells in shear thinning effect . . . . . . . . . . . 13

Figure 1.2 - Optimization approaches . . . . . . . . . . . . . . . . . . . 15

Figure 2.1 - Shear rate dependent viscosity for different models (logarithmic scale) 20

Figure 2.2 - Taylor-Hood mixed elements . . . . . . . . . . . . . . . . . . 23

Figure 3.1 - Topology optimization procedure for designing non-Newtonian fluid channels . . . . . . . . . . . . . . . 26

Figure $3.2-$ Fixed extended design domain . . . . . . . . . . . . . . . . 27

Figure 3.3 - Influence of penalty parameter . . . . . . . . . . . . . . . . . . . 29

Figure 5.1 - Topology optimization implementation flow chart . . . . . . . . . . . 43

Figure 5.2 - Approaches of computing the adjoint problem . . . . . . . . . . . . 46

Figure 6.1 - Backward facing step problem . . . . . . . . . . . . . . . . . . . . 49

Figure $6.2-$ Velocity profile for newtonian model. (a) $U_{\max }=0.025 \mathrm{~m} / \mathrm{s}$. (b) $U_{\max }=$

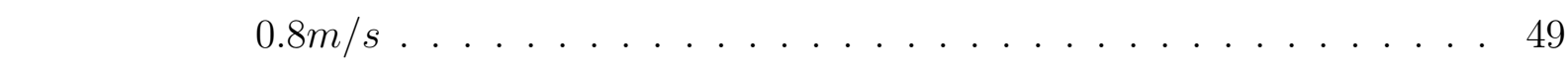

Figure 6.3 - Velocity profile for Carreau-Yasuda model. (a) $U_{\max }=0.025 \mathrm{~m} / \mathrm{s}$. (b)

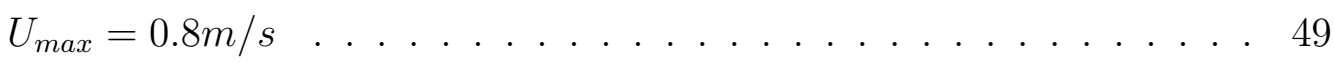

Figure $6.4-$ Velocity profile obtained in literature: (a) $U_{\max }=0.025 \mathrm{~m} / \mathrm{s}$ and (b) $U_{\max }=0.8 \mathrm{~m} / \mathrm{s} \ldots \ldots \ldots \ldots \ldots$

Figure 6.5 - Velocity profile at the outlet for different viscosity models obtained with FEniCS: (a) $U_{\max }=0.025 \mathrm{~m} / \mathrm{s}$ and (b) $U_{\max }=0.8 \mathrm{~m} / \mathrm{s}$. . . . . 50

Figure 6.6-Double channel problem . . . . . . . . . . . . . . 51

Figure 6.7 - Topologies for the double channel problem with Stokes formulation . 52

Figure 6.8 - Double channel problem with zero stress boundary condition at the outlet . . . . . . . . . . . . . . . . . . 52

Figure 6.9 - Topology results for double channel and Navier-Stokes Newtonian model 53

Figure 6.10 - Velocity fields for double channel and Navier-Stokes Newtonian model 53

Figure 6.11 - Topology results for double channel and Navier-Stokes non-Newtonian model (Carreau-Yasuda viscosity model) . . . . . . . . . . . . . . . 54

Figure 6.12 - Velocity fields for double channel and Navier-Stokes non-Newtonian model (Carreau-Yasuda viscosity model) . . . . . . . . . . . . . . . . 54

Figure 6.13 - Viscosity fields for double channel and Navier-Stokes non-Newtonian model (Carreau-Yasuda Viscosity Model) . . . . . . . . . . . . . . . . 54

Figure 6.14-Obstacle problem . . . . . . . . . . . . . . . . 55

Figure 6.15 - Optimized topologies for the obstacle problem . . . . . . . . . . . . 56

Figure 6.16 - Velocity and viscosity profiles for the Carreau-Yasuda model . . . . . 56

Figure 6.17 - Arterial bypass domain . . . . . . . . . . . . . . . . 57

Figure 6.18 - Initial guess for bypass topology optimization of fully blocked artery . 58 
Figure 6.19 - Velocity and viscosity fields for bypass initial guess of fully blocked

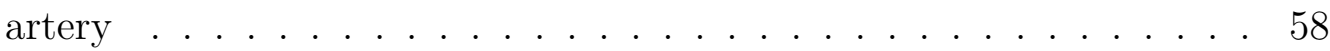

Figure 6.20 - Final topology for bypass optimization of fully blocked artery . . . . 59

Figure 6.21 - Velocity and viscosity fields for bypass optimization of fully blocked artery . . . . . . . . . . . . . . . . . . . 59

Figure 6.22-Convergence curve for bypass optimization of fully blocked artery . . 60

Figure 6.23 - Topology changes during bypass optimization of fully blocked artery . 60

Figure 6.24 - Design domain and boundary conditions for stenosed artery bypass . 61

Figure 6.25 - Final topology for stenosed artery optimization (Case 1) . . . . . . . 62

Figure 6.26 - Velocity field for stenosed artery optimization (Case 1) . . . . . . . . 62

Figure 6.27 - Viscosity field for stenosed artery optimization (Case 1) . . . . . . . . 63

Figure 6.28 - Final topology for stenosed artery optimization (Case 2) . . . . . . . 63

Figure 6.29 - Velocity field for stenosed artery optimization (Case 2) . . . . . . . . 64

Figure 6.30 - Viscosity field for stenosed artery optimization (Case 2) . . . . . . . . 64

Figure 6.31 - Final topology for stenosed artery optimization (Case 3) . . . . . . . 65

Figure 6.32 - Velocity field for stenosed artery optimization (Case 3) . . . . . . . . 65

Figure 6.33 - Viscosity field for stenosed artery optimization (Case 3) . . . . . . . . 65

Figure 6.34 - Gray final topology for pure vorticity optimization . . . . . . . . . . . 66

Figure 6.35 - Final topology for vorticity optimization . . . . . . . . . . . . . . 67

Figure 6.36 - Convergence curve for bypass optimization considering vorticity functional with added inertial part . . . . . . . . . . . . . . . . . 67

Figure 6.37 - Final topology considering the multi-objective functional . . . . . . . 68

Figure 6.38 - Convergence curve for optimization considering the multi-objective functional . . . . . . . . . . . . . . . . . . . . . . . . 68

Figure 6.39 - Topology changes during bypass optimization considering the multi-objective functional . . . . . . . . . . . . . . . . . . . . 69

Figure 6.40 - Design domain and boundary conditions for the 3D convergent diffuser 70

Figure 6.41 - Final topology for the 3D convergent diffuser optimization . . . . . . 70

Figure 6.42 - Velocity field for optimized 3D convergent diffuser . . . . . . . . . . . 71

Figure 6.43 - Viscosity field for optimized 3D convergent diffuser . . . . . . . . . . 71 


\section{LIST OF TABLES}

Table 6.1 - Carreau-Yasuda parameters for blood . . . . . . . . . . . . . . . . 48

Table 6.2 - Functional values for double channel . . . . . . . . . . . . . . . . 53

Table 6.3 - Final Functional values for bypass optimization of fully blocked artery 60

Table 6.4 - Cases division for bypass optimization of partial blocked artery . . . . 61

Table 6.5 - Functional values for vorticity optimization . . . . . . . . . . . . 66

Table 6.6 - Functional values for optimization considering the multi-objective functional . . . . . . . . . . . . . . . . . . . . . . . . . . . . . . . . . . 68 


\section{ACRONYMS}

CFD Computational Fluid Dynamics

PDE Partial Differential Equation

FEM Finite Element Method

LBB Ladyzhenskaya-Babuška-Brezzi

TOM Topology Optimisation Method

IPOpt Internal Point Optimizer

FFC FEniCS Form Compiler

UFL Unified Form Language

UFC Unified Form-Assembly Code

PETSc Portable, Extensible Toolkit for Scientific Computation

GMRES Generalized minimal residual

Bicstab Biconjugated Gradient Stabilized

MUMPS MUltifrontal Massively Parallel Sparse

ILU Incomplete Lower Upper 


\section{NOTATION}

$\begin{array}{ll}\mathbf{u} & \text { Velocity field } \\ p & \text { Pressure field } \\ \varrho & \text { Mass density } \\ \mu & \text { Dynamic viscosity } \\ \sigma & \text { Cauchy stress tensor } \\ \mathbf{f} & \text { Field forces } \\ I & \text { Identity tensor } \\ \epsilon & \text { Symmetric gradient } \\ \alpha & \text { Inverse permeability } \\ \dot{\gamma} & \text { Shear rate } \\ n & \text { Power law index } \\ \Omega & \text { Design domain } \\ \rho & \text { Design variable } \\ \Psi & \text { Velocity interpolation function } \\ \chi & \text { Pressure interpolation function } \\ x, y & \text { Global coordinates } \\ \xi, \eta & \text { Local element coordinates } \\ q & \text { Penalty parameter } \\ \mathbf{\Phi} & \text { Energy dissipation function } \\ \mathbf{J}_{\mathbf{s s}} & \text { Shear stress function } \\ \mathbf{J}_{\mathbf{v}} & \text { Vorticity function } \\ \Gamma & \text { Boundary condition } \\ \lambda^{*} & \text { Adjoint vector } \\ & \end{array}$




\section{CONTENTS}

$1 \quad$ INTRODUCTION $\ldots \ldots \ldots \ldots \ldots \ldots \ldots \ldots$

$1.1 \quad$ Non-Newtonian Blood Flow $\ldots \ldots \ldots \ldots$

1.2 Optimization Methods $\ldots \ldots \ldots \ldots \ldots$

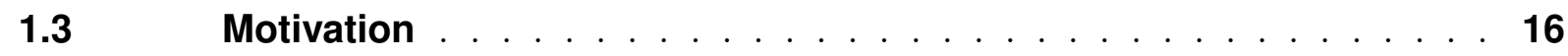

1.4 Objectives $\ldots \ldots \ldots \ldots \ldots \ldots \ldots$

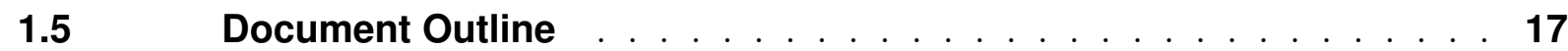

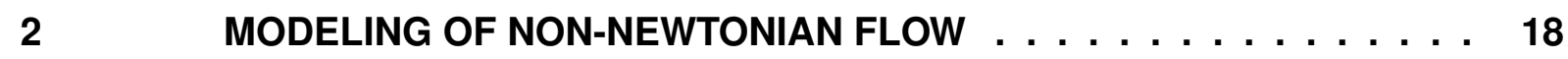

$2.1 \quad$ Navier-Stokes Equations $\ldots \ldots \ldots \ldots$

$2.2 \quad$ Non-Newtonian Effects $\ldots \ldots \ldots \ldots$

2.3 Finite Element Method . . . . . . . . . . . . . . . . . . 20

$2.3 .1 \quad$ Problem Formulation . . . . . . . . . . . . . . . . . 20

$2.3 .2 \quad$ Implemented Finite Element $\ldots \ldots \ldots \ldots$

$3 \quad$ TOPOLOGY OPTIMIZATION $\ldots \ldots \ldots \ldots \ldots \ldots$

$3.1 \quad$ Topology Optimization Applied to Fluid Flow $\ldots \ldots \ldots 26$

$3.2 \quad$ Extended Design Domain $\ldots \ldots \ldots \ldots$

$3.3 \quad$ Material Model $\ldots \ldots \ldots \ldots \ldots \ldots \ldots$

$3.4 \quad$ Problem Definition . . . . . . . . . . . . . . . . . 30

$3.4 .1 \quad$ Energy Dissipation . . . . . . . . . . . . . . . . . 30

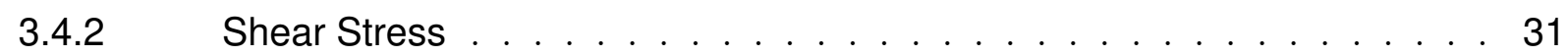

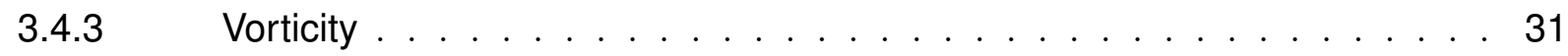

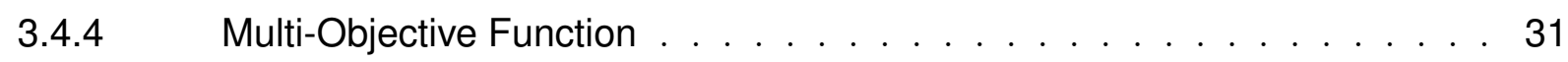

$3.4 .5 \quad$ Volume Constraint $\ldots \ldots \ldots \ldots \ldots$

$3.5 \quad$ Discrete Form . . . . . . . . . . . . . . . . . . . . 33

$4 \quad$ SENSITIVITIES CALCULATION $\ldots \ldots \ldots \ldots \ldots \ldots \ldots$

$4.1 \quad$ Adjoint Method . . . . . . . . . . . . . . . . . 35

$4.2 \quad$ Energy Dissipation Sensitivity Analysis $\ldots \ldots \ldots \ldots$

$4.2 .1 \quad$ Sensitivity of Viscosity $\ldots \ldots \ldots \ldots \ldots$

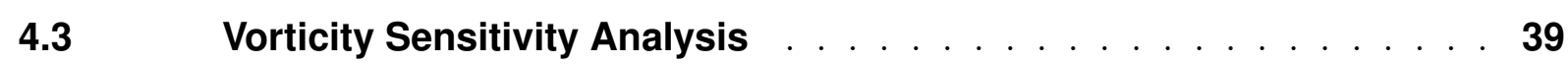

$4.4 \quad$ Shear Stress Sensitivity Analysis $\ldots \ldots \ldots . \ldots . \ldots 40$

$4.5 \quad$ Sensitivity Analysis of Multi-Objective Function $\ldots \ldots \ldots 41$

5 NUMERICAL IMPLEMENTATION $\ldots \ldots \ldots \ldots \ldots \ldots$

$5.1 \quad$ Optimization Algorithm $\ldots \ldots \ldots \ldots$ 
5.2 Software Environment . . . . . . . . . . . . . . . . . . . . . . . . 44

$5.2 .1 \quad$ Finite Element Method . . . . . . . . . . . . . . . . . . . . . . . . . . 44

$5.2 .2 \quad$ Sensitivities Computation . . . . . . . . . . . . . . . . . 45

$5.2 .3 \quad$ Optimizer . . . . . . . . . . . . . . . . . . 46

$6 \quad$ NUMERICAL RESULTS $\ldots \ldots \ldots \ldots \ldots$

$6.1 \quad$ FEM Verification . . . . . . . . . . . . . . . . . . . 48

$6.2 \quad$ Topology Optimization Results . . . . . . . . . . . . . . . . . . . 51

$6.2 .1 \quad$ Double Channel . . . . . . . . . . . . . . . . . . . . . . . . . . 51

6.2 .2 Obstacle . . . . . . . . . . . . . . . . . . . . . 55

$6.2 .3 \quad$ Arterial Bypass . . . . . . . . . . . . . . . . . . 57

$6.2 .4 \quad$ Bypass considering Vorticity . . . . . . . . . . . . . . . . 65

$6.2 .5 \quad$ Multi-objective bypass . . . . . . . . . . . . . . . . . . . . . 67

6.2 .6 3D Difuser . . . . . . . . . . . . . . . . . . . . . 69 69

7 CONCLUSIONS . . . . . . . . . . . . . . . . . . . 72

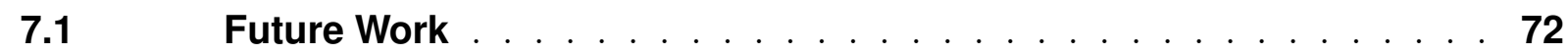

REFERENCES ....................... . . 73 


\section{INTRODUCTION}

\subsection{Non-Newtonian Blood Flow}

Flow of non-Newtonian fluids presents features that are not described by the linearly viscous Newtonian models with a constant value of viscosity. An important subclass of non-Newtonian fluids consists of those whose viscosity depends on the shear rate and/or on the pressure. These fluids play an important role in various areas of application, for instance, in chemical engineering, geology and also, blood rheology.

Blood is a concentrated suspension of red blood cells (erythrocytes), white blood cells (leukocytes), platelets (thrombocytes) in an aqueous polymer solution called plasma. The non-Newtonian effects of whole blood in viscometric flow are closely related to its microscopic structure (GIJSEN; VOSSE; JANSSEN, 1999), specially at low shear rates. The red blood cells are the dominant components in rheological behavior of blood, mainly due to a significantly higher concentration compared to the other formed cellular elements (ROBERTSON; SEQUEIRA; OWENS, 2009). Both shear thinning and viscoelasticity are related to aggregation, deformation and alignment of these cells, as shown in Fig 1.1.

Figure 1.1 - Influence of red blood cells in shear thinning effect.

(a) Equilibrium.

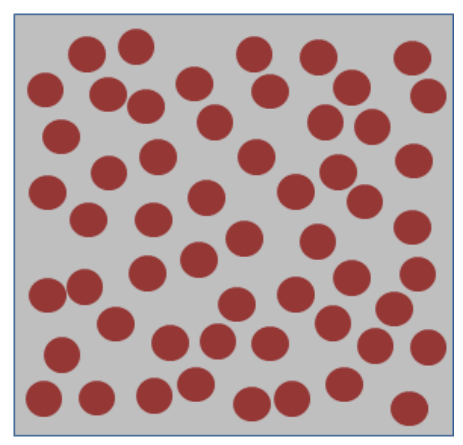

(b) Shear thinning effect.

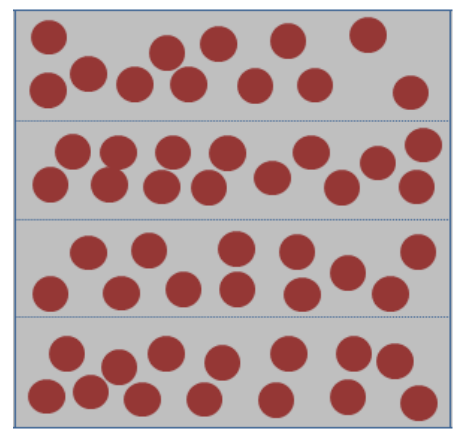

The study of blood flow plays an important role in disease research, treatment planning and design of medical devices. Some of the most relevant medical devices are arterial bypass grafts which provide solutions for diseases such as artherosclerosis by creating an alternative route around critically blocked arteries (FITZGIBBON et al., 1996) and ventricular assist devices (VADs) that can be an alternative for patients that present heart failure, specially as temporary solution as they await for a heart transplant (SLAUGHTER et al., 2013).

Since traditional laboratory experiments are costly and time demanding, Computational 
Fluid Dynamics (CFD) applied to fluid systems dealing with blood flow has been an active area of intensive research. Motivated by problems in biomedical engineering, many studies aim to find an appropriate numerical model of non-Newtonian effects in blood flow in order to obtain more accurate results (BOYD; BUICK; GREEN, 2007), (HUNDERTMARK-ZAUŠKOVÁ; LUKÁČOVÁ-MEDVID'OVÁ, 2010), (EVJU, 2011).

Numerical optimization procedures based on CFD present various advantages when compared to the traditional trial and error method. Thus, many approaches for the design of non-Newtonian fluidic systems have been developed.

In the work of Abraham, Behr and Heinkenschloss (2005), shape optimization was applied to efficient design of arterial bypass graft, with the goal of minimizing shear stress, which is related to field processes such as damage of red blood cells and platelet aggregation. In addition, the use of this optimization method for time-dependent domains was studied by Sokołowski and Stebel (2014). Sousa, Castro and António (2010) presented an optimization framework that is suited to perform shape optimization of complex geometries in compute-intensive applications by using an genetic algorithm approach.

This work in particular will model blood as a shear-thinning non-Newtonian fluid and apply the topology optimization method in order to design efficient channels for conducting blood, evaluating the model viscosity effects on the final topology.

\subsection{Optimization Methods}

An optimization algorithm is used to find an optimized solution, which can be obtained numerically to accelerate the design process. There are three basic approaches that can be used in numerical optimization: parametric optimization, shape optimization and topology optimization, as shown in Fig. 1.2. Each one of these approaches is more suitable for a type of application, according to the problem design objectives and restrictions. 
Figure 1.2 - Optimization approaches.

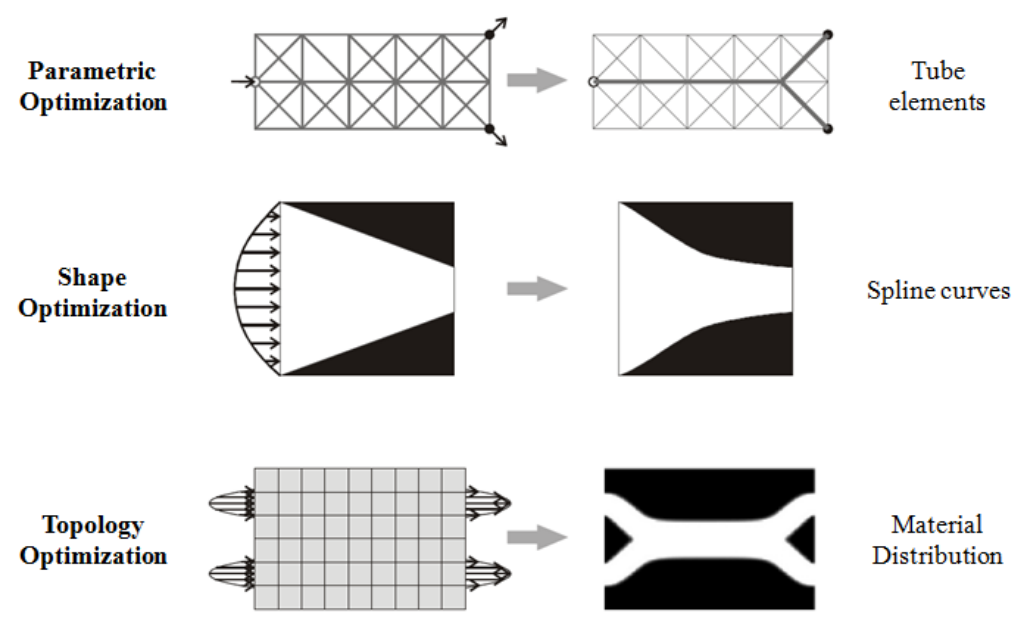

Adapted from Koga (2010).

In parametric optimization, also known as size optimization, the system geometry is previously defined and a basic model is described through some parameters that can be related to the geometry, material, physical properties, among others. The value of this parameters varies until a configuration considered optimized is reached. As an example, the work of Murakami and Mikic (2001) studies the optimization of multi-channel heat sinks for electronic devices and the channel diameter, number of channels and flow rate are considered as design parameters. This method presents more flexibility when it comes to the design variable, that is not necessarily related to the system geometry.

In shape optimization, the contour boundaries of the system are modified in the process, aiming to extremize a determined objective function. In this case, the geometry is fixed as defined in the initial condition, which means that no holes or connections can be included in the domain, however its shape is allowed to change. The work of Abraham, Behr and Heinkenschloss (2005) shows the optimization of an arterial bypass graft using this method, where the main goal is to reduce the total shear stress in blood flow.

In topology optimization method (BENDSOE; SIGMUND, 2003), an amount of material is distributed inside a domain, in a way of searching the optimal geometry of the system according to a desired characteristic. The use of this technique enables more complex and non-intuitive solutions to emerge. Thus, it is appropriate for applications where more freedom to modify the domain is desired. The material distribution over the domain is controlled by a design variable. The design variable of a topology optimization problem is used to determine the absence or presence of material within each point. The final topology presents a domain distribution described as "empty" or "filled" with material. In solid mechanics, the material model determines where the structure will be filled with solid material. However, in fluid systems a porous domain is used and the final domain shows which portions of the domain will be filled with fluid, and which will be filled with 
a low permeability, representing the "solid", indicating the absence of flow.

Recently, researchers have shown interest in applying the topology optimization method to devices conducting blood, considering non-Newtonian effects existent in this fluid. There are approaches that model the fluid flow through the Navier-Stokes equations, as proposed by Hyun, Wang and Yang (2014), Zhang and Liu (2015) and also through the Lattice Boltzmann method as shown in the work of Pingen and Maute (2010).

Thus, this work focuses in optimizing fluid blood flow devices, taking into account non-Newtonian effects in the dynamic viscosity, through the use of the numerical topology optimization procedure based on finite element method. The methodology is applied to design bi-dimensional channels, considering generic design domains.

\subsection{Motivation}

The study of non-Newtonian flow is important in bioengineering, to model correctly the flow of biological fluids. Among these, the most relevant are medical devices dealing with blood, such as bypass grafts and pumping systems, where the main goal is to reduce blood cell damage caused by artificial flows.

The use of computational simulation and optimization algorithms is a valid methodology to improve the design process of medical devices for blood flow, reducing its development time. This work studies the issues of blood constitutive behavior on the solution of the optimization design problem. The application of topology optimization to these systems makes it possible to obtain non-intuitive designs due to the freedom of domain modifications, allowing new borders and fluid regions to emerge.

\subsection{Objectives}

The present work aims to develop a methodology for designing optimized channels for non-Newtonian fluid flow, with emphasis on applications in bioengineering field of devices conducting blood, by using computer simulation and topology optimization method. The channels obtained should be efficient to conduct blood with low energy consumption and reduce damage to blood cells. Thus, the design problem requisites chosen aim to minimize energy dissipation, vorticity and shear stress. In order to achieve more accurate results, fluid flow is modeled by incompressible and steady state Navier-Stokes equations, coupled with a viscosity cross model, which takes into account non-Newtonian fluid effects. The system governing equations are solved numerically by using Finite Element Method (FEM). Topology optimization method is used to determine the path to be followed by fluid flow, by introducing a porosity model that allows the design variable to vary continuously from 
solid to fluid. This procedure is performed by a gradient based optimizer that uses the interior point method.

\subsection{Document Outline}

This document is organized as follows. In Chapter 2, the problem governing equations are presented and the algebraic system used in the finite element method is shown. In Chapter 3, the topology optimization method is described and the problem is defined in terms of its objective function and constraints. Furthermore, in Chapter 4, the sensitivity analysis by using the adjoint method is introduced. In Chapter 5 , it is explained how the numerical implementation is performed, detailing the optimization procedure and the software used at each optimization phase. In Chapter 6, the numerical results obtained are presented, comparing different viscosity models. Finally, in Chapter 7, some overall conclusions are reported and some suggestions of future work are presented. 


\section{MODELING OF NON-NEWTONIAN FLOW}

\subsection{Navier-Stokes Equations}

In this work, the fluid flow will be considered incompressible, laminar and in steady state regime. The system dimensions are chosen to be much larger than blood cells, so that fluid flow model based on the continuous assumption can be employed (ROBSON, 2003).

The fluid motion is described by the Navier-Stokes equations (BORRVALL; PETERSSON, 2003)

$$
\varrho\left(\frac{\partial \mathbf{u}}{\partial t}+\nabla \mathbf{u} \cdot \mathbf{u}\right)=\nabla \cdot \sigma+\mathbf{f}
$$

where $\mathbf{u}$ is the velocity field, $\varrho$ is the mass density and $\mathbf{f}$ are field forces and the Cauchy Stress Tensor $\sigma$ is defined as

$$
\sigma(\mathbf{u}, p)=2 \mu \epsilon(\mathbf{u})-p \mathbf{I}
$$

where $\mathbf{I}$ denotes the identity tensor, $p$ is the pressure, $\mu$ is the dynamic viscosity, and $\epsilon(\mathbf{u})$ is the symmetric gradient given by

$$
\epsilon(\mathbf{u})=\frac{1}{2}\left(\nabla \mathbf{u}+(\nabla \mathbf{u})^{T}\right)
$$

The mass conservation equation combined for incompressible flow ( $\varrho$ is constant) results in the following continuity equation

$$
\nabla \cdot \mathbf{u}=0
$$

Also, since the flow is assumed to be in steady state regime, the derivative $\frac{\partial \mathbf{u}}{\partial t}$ is null. Therefore, we have the Navier-Stokes equations for incompressible steady state flow.

$$
\begin{gathered}
\varrho(\nabla \mathbf{u} \cdot \mathbf{u})=\nabla \cdot 2 \mu \epsilon(\mathbf{u})-\nabla p+\mathbf{f} \\
\nabla \cdot \mathbf{u}=0
\end{gathered}
$$

The topology optimization method distributes regions of solid and fluid through a domain, which makes it necessary to work with a model that is able to combine fluid and 
solid behavior. A porous material model is used to simulate the solid regions by imposing local flow restrictions. The flow through a porous media is described by Darcy's Law (BORRVALL; PETERSSON, 2003):

$$
\alpha \mathbf{u}=\nabla p-\mathbf{f}
$$

where $\alpha$ is the inverse permeabilty.

This parameter $\alpha$ allowsto differentiate low permeability areas $(\alpha \rightarrow \infty)$, where the flow is blocked, representing solid material, from high permeability areas $(\alpha \approx 0)$ where there is unconstrained fluid flow. This material model of flow in porous media is used in topology optimization problems for both newtonian fluids (BORRVALL; PETERSSON, 2003) and non-newtonian (PINGEN; MAUTE, 2010).

Brinkman's equation is obtained by introducing a porosity term from Darcy's Law into Navier-Stokes, given by (HYUN; WANG; YANG, 2014):

$$
\varrho(\mathbf{u} \cdot \nabla \mathbf{u})=-\nabla p+\nabla \cdot 2 \mu \epsilon(\mathbf{u})+\mathbf{f}-\alpha \mathbf{u}
$$

\subsection{Non-Newtonian Effects}

Blood is composed by cellular elements suspended in an aqueous solution called plasma. The cellular elements are red blood cells or erythrocytes, white blood cells or leukocytes and platelets or thrombocytes. Due to its microscopic structure, this fluid is more accurately represented when modeled as non-Newtonian fluid, where the dynamic viscosity $\mu$ is dependent of the shear rate $\dot{\gamma}$, given by the following equation:

$$
\dot{\gamma}=\sqrt{2 \epsilon(\mathbf{u}): \epsilon(\mathbf{u})}
$$

where the symbol : stands for the double scalar product of two tensors.

When related to viscosity effects, non-Newtonian fluids can have two different behaviors. If the viscosity increases with shear rate, the fluid is called dilatant or shear-thickening. If the effective viscosity decreases with shear, then it is called pseudoplastic or shear-thinning, which is the case of blood. In this work, fluids that present viscoelastic effects will not be considered.

Blood shear-thinning behavior is mainly related to following aspects of red blood cells: the propensity to form microstructures called rouleaux at low shear rates, their deformability, and the flow field alignment at high shear rates (ROBERTSON; SEQUEIRA; OWENS, 2009).

A very common model to describe the shear rate dependent viscosity is the Power-Law 
equation

$$
\mu(\dot{\gamma})=K \dot{\gamma}^{n-1}
$$

where $K$ is a positive constant and $n$ is the Power-Law index, which defines the behaviour of the fluid. A Newtonian fluid presents $n=1$, whereas for $n>1$, the fluid is shear-thickening and for $n<1$, it is shear-thinning (BOEK; CHIN; COVENEY, 2003).

Since the Power-law model applied to shear-thinning fluids presents an infinite dynamic viscosity at very low shear rates and zero viscosity at very high shear rates, which can lead to numerical instabilities, another model is adopted.

The blood viscosity model implemented is the Carreau-Yasuda, which given by Equation 2.11

$$
\mu(\dot{\gamma})=\mu_{\infty}+\left(\mu_{0}-\mu_{\infty}\right)\left[1+(\lambda \dot{\gamma})^{a}\right]^{\frac{n-1}{a}}
$$

where $\lambda, a, n, \mu_{\infty}, \mu_{0}$ are fluid parameters.

Thus, for zero shear rate, the viscosity presents a maximum value of $\mu_{0}=0.16$ Pa.s and for very high shear rates (approximately $>10^{3} \mathrm{~s}^{-1}$ ) the viscosity value converges to the same as the Newtonian model $\mu_{\infty}=0.0035$ Pa.s, as verified experimentally (MACOSKO, 1994). The relation between viscosity and shear rate for blood in the models discussed are presented in Figure 2.1.

Figure 2.1 - Shear rate dependent viscosity for different models (logarithmic scale).

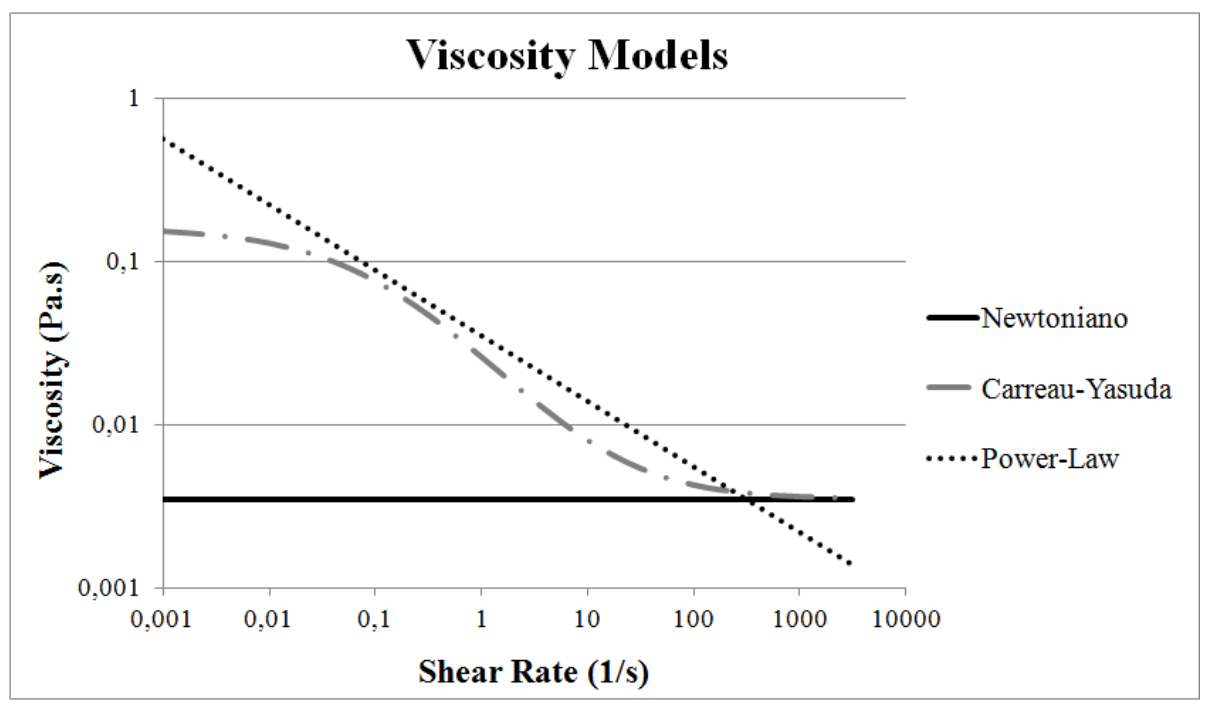

\subsection{Finite Element Method}

\subsubsection{Problem Formulation}

The partial differential equations (PDE) presented in Section 2.1 are solved by using a numerical method, since it is very difficult to solve them analytically. The 
finite element method (FEM) is implemented to find the approximate solution of the Navier-Stokes equations. This method is especially attractive for problems dealing with complex geometries, where other numerical methods are difficult to apply.

The first step for the FEM formulation is to obtain the weak formulation. For this, a weighted residual procedure is applied. The PDE is multiplied by a test function and the resulting equation is integrated over the domain $\Omega$. The Galerkin's method is used, where the weighting functions and the unknown fields are represented by the same space functions. Thus, the stated problem weak formulation is given by (ROMERO; SILVA, 2014)

$$
\begin{gathered}
\int_{\Omega} \alpha \mathbf{u} \cdot \mathbf{v} d \Omega+\int_{\Omega} \varrho(\mathbf{u} \cdot \nabla \mathbf{u}) \cdot \mathbf{v} d \Omega+\int_{\Omega} \sigma: \nabla \mathbf{v} d \Omega=\int_{\Omega} \mathbf{f} \cdot \mathbf{v} d \Omega \\
\int_{\Omega} q(\nabla \cdot \mathbf{u}) d \Omega=0
\end{gathered}
$$

where $\mathbf{v}$ and $q$ are weighting test functions, with $q$ being scalar and $\mathbf{v}$ a vector.

The stress tensor $\sigma$ is defined by:

$$
\sigma=\left[\begin{array}{cc}
-p+2 \mu \frac{\partial u_{1}}{\partial x_{1}} & \mu\left(\frac{\partial u_{1}}{\partial x_{2}}+\frac{\partial u_{2}}{\partial x_{1}}\right) \\
\mu\left(\frac{\partial u_{1}}{\partial x_{2}}+\frac{\partial u_{2}}{\partial x_{1}}\right) & -p+2 \mu \frac{\partial u_{2}}{\partial x_{2}}
\end{array}\right]
$$

The Finite Element Method is used to find an approximated solution for the weak formulation problem, through the discretization of the continuous model, where the solution is evaluated at discrete points over the domain, called nodes. The relation between approximated continuous distribution of velocity and pressure and the nodal values, per element, is given by:

$$
\begin{gathered}
u_{1} \cong \sum_{j=1}^{n} \psi_{j} u_{j}^{1}=\mathbf{\Psi}^{T} \mathbf{u}_{\mathbf{1}} ; u_{2} \cong \sum_{j=1}^{n} \psi_{j} u_{j}^{2}=\mathbf{\Psi}^{T} \mathbf{u}_{\mathbf{2}} \\
p \cong \sum_{j=1}^{m} \chi_{j} p_{j}=\chi^{T} \mathbf{p}
\end{gathered}
$$

where $\mathbf{u}=\left[u_{1} u_{2}\right]^{T}$ is velocity field, $p$ is the pressure, $\mathbf{u}_{\mathbf{1}}, \mathbf{u}_{\mathbf{2}}, \mathbf{p}$ are vectors at the nodal points of the approximated solutions, $\chi$ and $\boldsymbol{\Psi}$ are interpolation functions defined in Section 2.3 .2 , $n$ and $m$ are the number of interpolations nodes per element for velocity and pressure, respectively.

By replacing the approximation for pressure and velocity fields obtained through finite element interpolation functions in the week formulation, we obtain the algebraic equations 
for the finite element method. The matricial coefficients per element are given by:

$$
\begin{aligned}
\mathbf{K}_{\alpha} & =\int_{\Omega^{e}} \alpha \boldsymbol{\Psi} \boldsymbol{\Psi}^{T} d x ; \\
\mathbf{C}_{j}\left(\mathbf{u}_{j}\right) & =\int_{\Omega^{e}} \varrho \boldsymbol{\Psi}\left(\boldsymbol{\Psi}^{T} \mathbf{u}_{i}\right) \frac{\partial \boldsymbol{\Psi}}{\partial x_{j}} d x \\
\mathbf{K}_{i j} & =\int_{\Omega^{e}} \mu \frac{\partial \boldsymbol{\Psi}}{\partial x_{j}} \frac{\partial \boldsymbol{\Psi}^{T}}{\partial x_{i}} d x ; \\
\mathbf{Q}_{i} & =\int_{\Omega^{e}} \frac{\partial \boldsymbol{\Psi}}{\partial x_{i}} \chi^{T} d x \\
\mathbf{f}_{i} & =\int_{\Omega^{e}} \boldsymbol{\Psi} f_{i} d x, \quad i, j=1,2 ;
\end{aligned}
$$

The global algebraic system, considering two-dimensional case, is defined in the matrix form as (ROMERO; SILVA, 2014):

$$
\begin{array}{r}
{\left[\begin{array}{ccc}
\mathbf{K}_{\alpha} & 0 & 0 \\
0 & \mathbf{K}_{\alpha} & 0 \\
0 & 0 & 0
\end{array}\right]\left\{\begin{array}{l}
\mathbf{u}_{1} \\
\mathbf{u}_{2} \\
\mathbf{p}
\end{array}\right\}+\left[\begin{array}{ccc}
\mathbf{C}_{1}\left(\mathbf{u}_{1}\right)+\mathbf{C}_{2}\left(\mathbf{u}_{2}\right) & 0 & 0 \\
0 & \mathbf{C}_{1}\left(\mathbf{u}_{1}\right)+\mathbf{C}_{2}\left(\mathbf{u}_{2}\right) & 0 \\
0 & 0 & 0
\end{array}\right]\left\{\begin{array}{l}
\mathbf{u}_{1} \\
\mathbf{u}_{2} \\
\mathbf{p}
\end{array}\right\}+} \\
{\left[\begin{array}{ccc}
2 \mathbf{K}_{11}+\mathbf{K}_{22} & \mathbf{K}_{12} & -\mathbf{Q}_{1} \\
\mathbf{K}_{21} & \mathbf{K}_{11}+2 \mathbf{K}_{22} & -\mathbf{Q}_{2} \\
-\mathbf{Q}_{1}^{T} & -\mathbf{Q}_{2}^{T} & 0
\end{array}\right]\left\{\begin{array}{l}
\mathbf{u}_{1} \\
\mathbf{u}_{2} \\
\mathbf{p}
\end{array}\right\}=\left\{\begin{array}{l}
\mathbf{f}_{1} \\
\mathbf{f}_{2} \\
\mathbf{0}
\end{array}\right\}}
\end{array}
$$

The resulting system is non-symmetrical and non-linear due to the term $\mathrm{C}(\mathrm{u})$. Therefore, to solve the system a non-linear solver, based on an iterative Newton method, needs to be applied (GERSBORG-HANSEN; SIGMUND; HABER, 2005). The velocity and pressure fields are the state variables of the problem, and the residual can be defined in a compact form based on Equation 2.19 as

$$
\mathbf{R}(\mathbf{z}(\rho), \rho)=0
$$

where $\mathbf{z}$ is a vector that contains all state variables $\mathbf{z}=\left[u_{1} u_{2} p\right]^{T}$, and it is shown that $\mathbf{R}$ and state variables $\mathbf{z}$ depend on the material model described in Section 3.3 .

The viscosity dependent terms would also introduce non-linearities to the system, which would lead to an increase of complexity. Thus, the viscosity is calculated with respect to the computed velocity field from the previous iteration. This approach of neglecting the non-linear influence of viscosity has shown to be satisfactory, and has been used by Abraham, Behr and Heinkenschloss (2005) where the derivative of $\mu$ with respect to $u$ is dropped. 


\subsubsection{Implemented Finite Element}

The choice of the problem discretization for the incompressible Navier-Stokes equations must satisfy the Ladyzhenskaya-Babuška-Brezzi (LBB or inf-sup) conditions (LOGG et al. 2012) in order to obtain a stable configuration. The use of equal-order continuous Lagrange finite element for both pressure and velocity leads to an unstable problem (ABRAHAM; BEHR; HEINKENSCHLOSS, 2005).

One of the most widely used types of element for solving Navier-Stokes equations that satisfy these LBB conditions is the Taylor-Hood element (TAYLOR; HOOD, 1973). It consists of a continuous polynomial interpolation function of degree $r \geq 2$ for the velocity components and a continuous polynomial of degree $r-1$ for the pressure field.

The finite element used in this work is a triangular Taylor-Hood element, where the velocity has a quadratic interpolation function with six nodes per element and the pressure has a linear interpolation with three nodes. Thus, in Equations 2.15 and 2.16, the sum indexes values are $n=6$ and $m=3$. For the topology optimization process, the design variable is defined to be constant within an element, as shown in the Figure 2.2

Figure 2.2 - Taylor-Hood mixed elements.

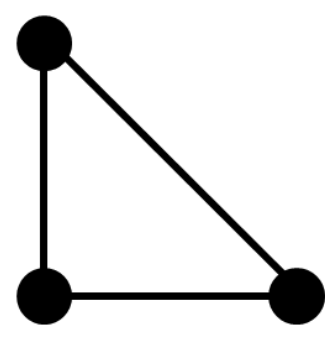

(a) Pressure

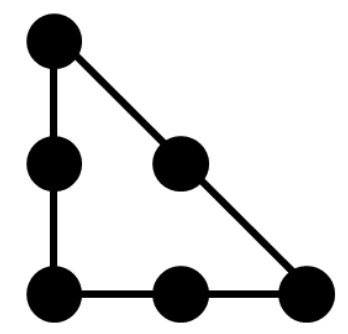

(b) Velocity

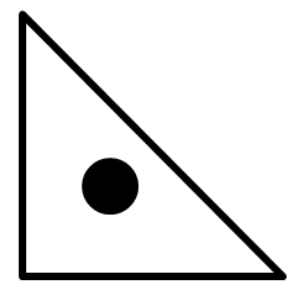

(c) Design Variable

The interpolation values are calculated in local normalized coordinates $\xi$ and $\eta$ $(0 \leq \xi \leq 1$ and $0 \leq \eta \leq 1)$ and then converted to the global coordinates, according to the following coordinate transformation for a triangular element with vertices $\left(x_{1}, y_{1}\right),\left(x_{2}, y_{2}\right)$ and $\left(x_{3}, y_{3}\right)$

$$
\begin{aligned}
& x=x_{1}+\left(x_{2}-x_{1}\right) \xi+\left(x_{3}-x_{1}\right) \eta \\
& y=y_{1}+\left(y_{2}-y_{1}\right) \xi+\left(y_{3}-y_{1}\right) \eta
\end{aligned}
$$

The linear pressure interpolation is given by:

$$
\begin{aligned}
& \chi_{1}(\xi, \eta)=1-\xi-\eta \\
& \chi_{2}(\xi, \eta)=\xi \\
& \chi_{3}(\xi, \eta)=\eta
\end{aligned}
$$


and the quadratic velocity interpolation in the local coordinates yields:

$$
\begin{aligned}
& \psi_{1}(\xi, \eta)=(1-\xi-\eta)(1-2 \xi-2 \eta) \\
& \psi_{2}(\xi, \eta)=\xi(2 \xi-1) \\
& \psi_{3}(\xi, \eta)=\eta(2 \eta-1) \\
& \psi_{4}(\xi, \eta)=4 \xi(1-\xi-\eta) \\
& \psi_{5}(\xi, \eta)=4 \xi \eta \\
& \psi_{6}(\xi, \eta)=4 \eta(1-\xi-\eta)
\end{aligned}
$$




\section{TOPOLOGY OPTIMIZATION}

The topology optimization (BENDSOE; SIGMUND, 2003) is a method that aims to design an optimized topology of a defined system in order to extremize a certain characteristic, which is represented by an objective function. The basic concept of this optimization type is that material can be distributed inside a domain, independent of the initial system configuration, by defining which domain point should be filled or empty. This method was originally developed for solving structural solid mechanics problems (BENDSOE; KIKUCHI, 1988), (SUZUKI; KIKUCHI, 1991) aiming to minimize mean compliance, where this distribution indicates presence or absence of solid material. In this specific work, where the fluid flow properties are evaluated, the filled parts represent fluid material and the empty parts represent solid that defines the contours of the fluid channel (BORRVALL; PETERSSON, 2003).

The implementation of topology optimization arises from the combination of an optimization algorithm with an analysis method. The analysis is usually performed by using Finite Element Method, which allows working with complex geometries.

The design variable, the parameter that will change through the optimization iterations, is defined by the model adopted to define the material distribution. Then, the design domain is specified where it is desired to obtain an optimized topology, with its boundary conditions. It is also necessary to define the objective function that will be extremized along with the constraints that the system must satisfy.

After the complete description of the continuous problem, it is written in its discrete form in order to execute the analysis step through the Finite Element Method. The optimization algorithm changes the system topology until a convergence criteria is reached. Then, the results are post-processed to smooth the edges and it is verified if the system requirements are still consistent before prototype building (Sá, 2016).

Figure 3.1 shows a simplified scheme of the main phases of design using topology optimization. 
Figure 3.1 - Topology optimization procedure for designing non-Newtonian fluid channels.
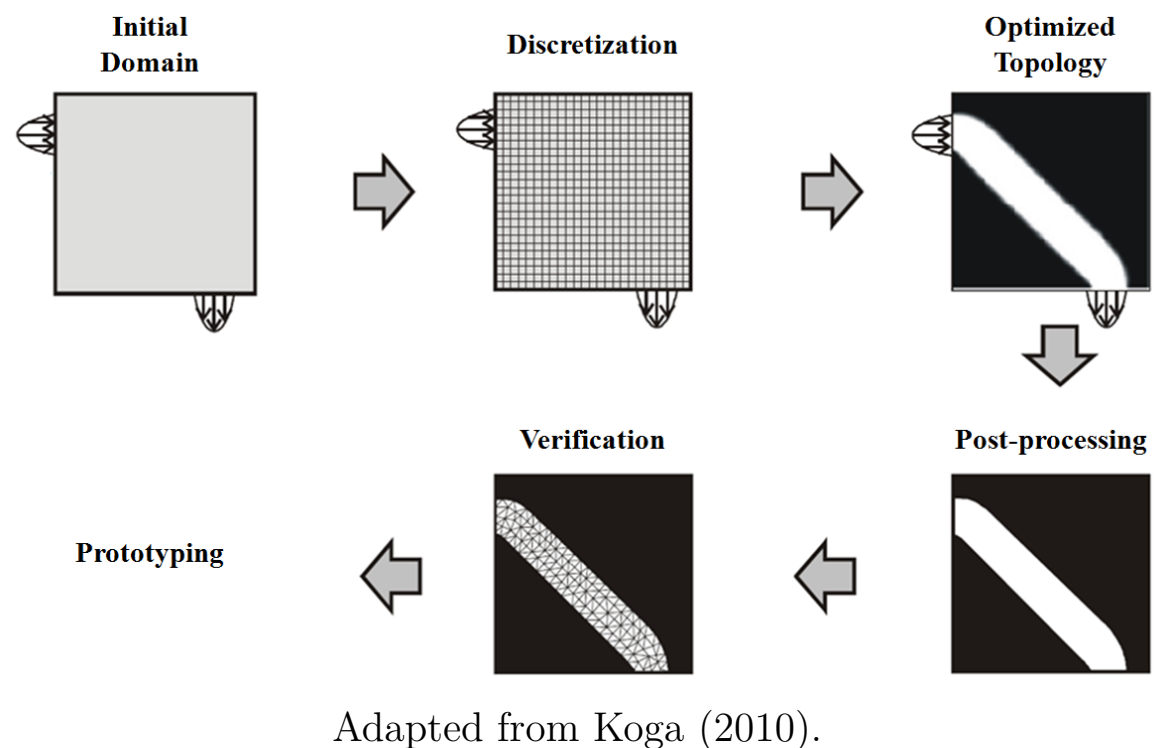

Adapted from Koga (2010).

\subsection{Topology Optimization Applied to Fluid Flow}

The implementation of topology optimization method (TOM) to fluid systems has been first introduced by Borrvall e Peterson, where it is applied to two-dimensional flow channel design aiming to minimize viscous energy dissipation (BORRVALL; PETERSSON, 2003). Fluid flow is modeled by considering steady state Stokes flow. The adopted design variable is the material pseudo-density.

Since then, this theme has been quite explored in literature. Non-linear effects that occur for higher Reynolds numbers and Navier-Stokes constitutive equations (GERSBORG-HANSEN; SIGMUND; HABER, 2005), large scale flow and three-dimensional problems (AAGE et al. 2008) and flows driven by body forces (DENG; LIU; WU, 2013) have been studied.

In the work of Klarbring et al. (2003), procedures from truss topology optimization are applied to flow networks, related to arterial tree-type networks.

Different methods for implementing topology optimization applied to fluid problems have been proposed, such as using the viscosity as design variable (EVGRAFOV, 2005) and also modelling the flow using the Lattice- Boltzmann method (PINGEN; EVGRAFOV; MAUTE, 2007).

The topology optimization method has been applied to systems with Stokes and Darcy flows coupled, by distributing regions of fluid and regions of porous material, which introduces the need for a regularization method (WIKER; KLARBRING; BORRVALL, 2007).

Recently, researchers have shown interest in applying the topology optimization method 
to design devices that conduct blood, by considering the non-Newtonian effects present in this fluid. One of the approaches, proposed by Hyun, Wang and Yang (2014), optimizes the fluid flow channel through the Navier-Stokes equations, where a multi-objective function is defined by relating reduction of wall shear stress and viscous energy dissipation through the use of different weights.

Another approach can be found in the work of Pingen and Maute (2010), who has implemented the optimization of bidimensional channel design aiming to reduce the average pressure drop between inlet and outlet, modelling the non-Newtonian fluid through the Lattice Boltzmann method.

Arterial bypass configurations problems with the objective of minimizing flow shear stress have been studied in the work of Zhang and Liu (2015). The topology optimization is implemented by using a level set method. The results are compared to the results of Abraham, Behr and Heinkenschloss (2005), that are achieved by using shape optimization.

\subsection{Extended Design Domain}

An important concept in topology optimization is the extended design domain, which consists in a predetermined fixed region in which the final system configuration must be contained. Within this fixed domain $\Omega$, the optimal solution $\Omega_{f}$, which represents the distribution of fluidic regions, must be found. Thus, the optimization algorithm works with a domain that is larger than the expected optimal solution. Figure 3.2 illustrates this concept.

Figure 3.2 - Fixed extended design domain.
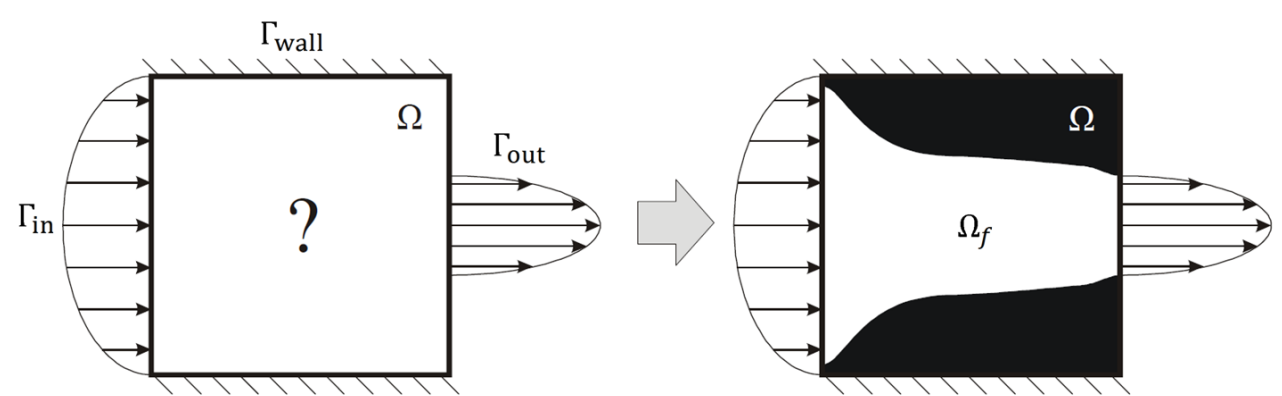

The main advantage of using a fixed domain, since its dimensions remain unchanged, is that the finite element mesh does not need to be readjusted during the optimization process, which makes the numerical implementation easier. 


\subsection{Material Model}

Another essential concept to Topology Optimization Method is the material model used which relax the discrete (0-1) problem in order to achieve an optimized solution. In this case, the optimization algorithm uses a density based model.

Given a domain $\Omega$ with predefined boundary conditions, it is desired to find in which regions of $\Omega$ must exist fluid and which must be solid in order to minimize (or maximize) a certain objective function subjected to a constraint of an amount of fluid.

At the final optimization result, the domain distribution should be discrete, i.e, the value of the design variable at each point is zero (solid) or one (fluid). Since the discrete topology optimization problem is bad posed, it is usual to work with intermediate values during the optimization process (WIKER; KLARBRING; BORRVALL, 2007). Thus, a material model is applied to relax the problem and allow a continuous transition between solid and fluid.

In the adopted material model, the design variable is the pseudo-density $\rho, 0 \leq \rho \leq 1$, which represents the amount of fluid present at each point of the domain. The material model represents a porous material that allows a continuous transition between these two states, through the inverse permeability term, present in Darcy's equation, as presented in Section 2.1.

In the final optimized topology, the design variable should achieve discrete values so that $\rho=1$ represents a pure fluid and $\rho=0$ indicates a fully solid material. In order to suppress the intermediate values of the pseudo-density $\rho$, non-linear interpolation functions are adopted for describing the inverse permeability as functions of $\rho$ (BORRVALL; PETERSSON, 2003), given by

$$
\alpha(\rho)=\alpha_{s}+\left(\alpha_{f}-\alpha_{s}\right) \frac{\rho(1+q)}{\rho+q}
$$

where $q$ is the penalty parameter that controls the linearity of the interpolation function, $\alpha_{f}=0$ is the inverse permeability for fluid material and $\alpha_{s} \rightarrow \infty$ is for solid material.

The influence of the penalty parameter is shown in Figure 3.3, where higher values lead to an interpolation function with linear behavior and the distribution of $\rho$ in the domain tends to find extreme values (0 or 1 ). Due to non-convexity, as stated by Borrvall and Petersson (2003), when a high value of $q$ is used there might be local optimal solution problems. Thus, the optimization is first solved with a small value of $q=0.01$ to obtain a better initial guess for the problem and then the penalty parameter value is increased in order to achieve a discrete solution. 
Figure 3.3 - Influence of penalty parameter.

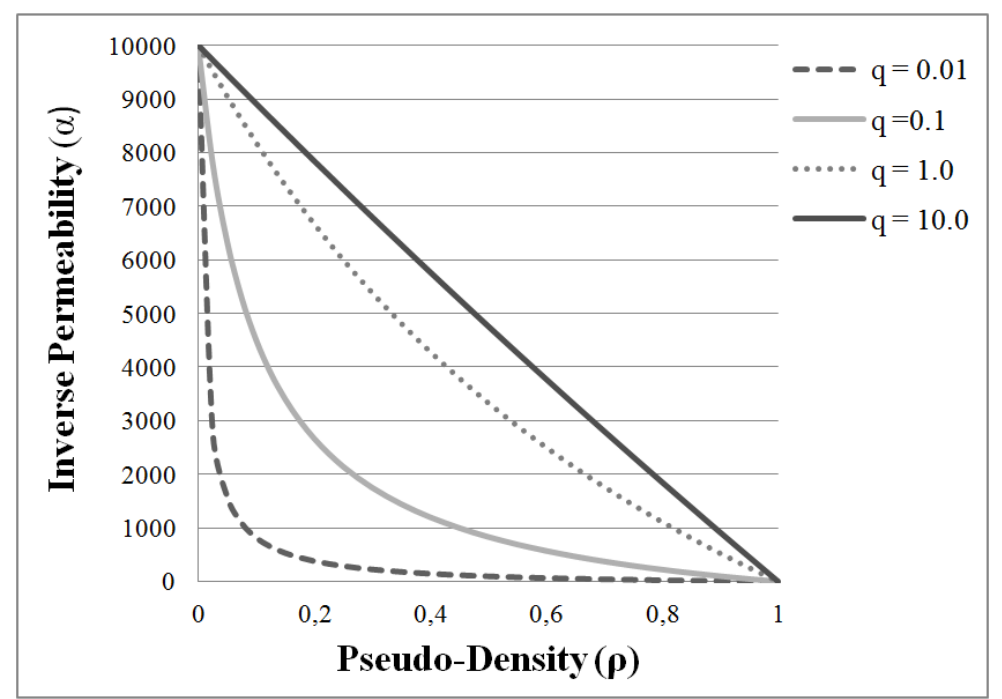

Some authors suggest that the non-Newtonian effects should be reduced in areas of high porosity in order to avoid convergence problems. Thus, the viscosity also is interpolated as a function of the pseudo-density $\rho$. Hyun, Wang and Yang (2014) proposed a model where viscosity is interpolated with the same penalty parameter $q$ used for the inverse permeability, given by

$$
\mu(\rho)=\mu_{s}+\left(\mu_{f}-\mu_{s}\right) \frac{\rho(1+q)}{\rho+q}
$$

where $\mu_{f}$ is the shear rate dependent viscosity $\mu(\dot{\gamma})$ and $\mu_{s}$ is equal to the Newtonian viscosity $\left(\mu_{\infty}=0.0035\right.$ Pa.s $)$.

Pingen and Maute (2010) introduced a scaling term $(1-\rho)^{K}$, where $K$ is a constant, into the Carreau-Yasuda viscosity model defined in Equation 2.11, resulting in

$$
\mu(\dot{\gamma}, \rho)=\mu_{\infty}+\left(\mu_{0}-\mu_{\infty}\right)(1-\rho)^{K}\left[1+(\lambda \dot{\gamma})^{a}\right]^{\frac{n-1}{a}}
$$

In both equations presented, when $\rho=1$, the material is pure fluid and presents a full non-Newtonian behavior and when $\rho=0$, the material is solid and the viscosity value adopted is numerically equal to the Newtonian viscosity $\mu_{\infty}$, which can be found in regions of high shear rate. This interpolation can be justified by the fact that highly porous or solid material is concentrated in the interfaces between fluid and solid, where the velocity gradients are higher and present a minimal value of viscosity, as shown in Section 2.2, and in regions where fluid flow is approximately null and can be neglected. 


\subsection{Problem Definition}

In an optimization problem, it is necessary to define the objective function, which represents the value that must be extremized, the constraints that the problem should satisfy and the design domain with its boundary conditions. The different objective functions studied will be presented next and the design domain and constraints will be detailed for each case in Section 6 .

The optimization problem in this work is defined by the following formulation

$$
\begin{aligned}
\text { Minimize : } & c(\mathbf{z}(\rho), \rho) \\
\rho & \\
\text { subjected to : } & \mathbf{g}(\mathbf{z}(\rho), \rho) \leq 0 \\
& \mathbf{r}(\mathbf{z}(\rho), \rho)=0 \\
& \rho_{\min } \leq \rho \leq \rho_{\max }
\end{aligned}
$$

where $c(\mathbf{z}(\rho), \rho)$ is a multi-objective function that can include terms of energy dissipation, shear stress and vorticity and $g(\mathbf{z}(\rho), \rho)$ is the volume constraint, described as follows. The term $r(\mathbf{z}(\rho), \rho)$ represents the problem governing equations defined in Section 2.1 and the limits of the design variable, according with the material model presented are $\rho_{\min }=0$ and $\rho_{\max }=1$.

\subsubsection{Energy Dissipation}

Minimizing the energy dissipation is one of the objective functions in this work. For this case, there are no forces applied to the fluid and the fluid velocities are normal to the boundaries, thus, to minimize the potential energy dissipation is equivalent to minimize the channel average pressure drop (BORRVALL; PETERSSON, 2003).

This objective function is valid in many applications, and it is specially important in applications where the energy available is restricted, requiring a good performance of the channel in which the fluid flows.

Borvall and Petersson proposed a formulation for the fluidic energy dissipation that considers the effect of material model, given by

$$
\Phi=\int_{\Omega} \frac{1}{2} \mu\left(\nabla \mathbf{u}+\nabla \mathbf{u}^{T}\right):\left(\nabla \mathbf{u}+\nabla \mathbf{u}^{T}\right) d \Omega+\int_{\Omega} \alpha(\rho) \mathbf{u}^{2} d \Omega
$$

The first term in the equation corresponds to the viscous dissipation effects present in the fluid flow. The second term corresponds to energy dissipation due to porous media model and it is introduced to minimize flow through porous and solid regions. 


\subsubsection{Shear Stress}

One important criterion for designing devices for blood flow is to minimize blood damage. Since shear stress in the flow field is related to hemolysis (damage to red blood cells) and it also influences processes as platelet aggregation and thrombus formation (PROBST et al., 2010), it can be a good indicator of quality in a biomedical application.

A common criteria used in blood flow problems is wall shear stress, which is typically defined on the channel wall. During the optimization process, the interface between solid and fluid changes continuously, which makes it difficult to evaluate this parameter.

Topology optimization authors usually consider the shear rate as a representative measure of shear stress. Thus, we aim to minimize the integral of the squared shear rate as an objective function ABRAHAM; BEHR; HEINKENSCHLOSS, 2005)

$$
J_{s s}=\int_{\Omega} 2 \epsilon(u): \epsilon(u) d \Omega
$$

where $\epsilon(u)$ is defined in Equation 2.3 .

It is important to notice that the only difference between this objective function and the previously defined energy dissipation objective function is the absence of the dynamic viscosity term.

\subsubsection{Vorticity}

High vorticity is prevalent in turbulent flow and can damage particles flowing in the current, causing a loss of material integrity, due to fluid mixing. It can be hazardous to sensitive fluids such as blood.

A measure of vorticity can be expressed in the functional given by (QUARTERONI; ROZZA, 2003; ABRAHAM; BEHR; HEINKENSCHLOSS, 2004):

$$
J_{v}(\mathbf{u})=\int_{\Omega}|\nabla \times \mathbf{u}|^{2} d \Omega=\int_{\Omega}\left(\frac{\partial u_{2}}{\partial x_{1}}-\frac{\partial u_{1}}{\partial x_{2}}\right)^{2} d \Omega
$$

As it is presented in the Results Chapter 6, the energy dissipation due to porous media model term, presented in Eq.3.5, is introduced in order to achieve discrete results.

$$
J_{v}(\mathbf{u})=\int_{\Omega}|\nabla \times \mathbf{u}|^{2} d \Omega+\int_{\Omega} \alpha(\rho) \mathbf{u}^{2} d \Omega=\int_{\Omega}\left(\frac{\partial u_{2}}{\partial x_{1}}-\frac{\partial u_{1}}{\partial x_{2}}\right)^{2} d \Omega+\int_{\Omega} \alpha(\rho) \mathbf{u}^{2} d \Omega
$$

\subsubsection{Multi-Objective Function}

In the present work, we combine the previous different objective functions to define a multi-objective function based on the weighting sum method, according to the following 
equation

$$
\Psi=\omega_{e} \Phi+\omega_{v} J_{v}+\omega_{s} J_{s s}
$$

where $\Phi$ is the viscous energy dissipation term, $J_{v}$ is related to vorticity and $J_{s s}$ represents total shear stress. $\omega_{e}, \omega_{v}$ and $\omega_{s}$ are the weighting coefficients associated with energy dissipation, vorticity and total shear stress respectively. Note that the sum $\omega_{e}+\omega_{v}+\omega_{s}=1.0$.

The functionals of the multi-objective function presented in Equation 3.9 have different orders of magnitude. Thus, in order to smooth these differences, two coefficients can be introduced (ZHU; ZHANG; FATIKOW, 2014):

$$
\begin{gathered}
\beta^{k}=\frac{\Phi^{k-1}}{J_{v}^{k-1}}, \quad k \geq 1 \\
\gamma^{k}=\frac{\Phi^{k-1}}{J_{s s}^{k-1}}, \quad k \geq 1
\end{gathered}
$$

where $\beta_{k}$ and $\gamma_{k}$ are weighting factors which values change at each iteration $k$ of the optimization algorithm, calculated with respect the previous iteration $k-1$. For $k=0$ the functional values for the initial domain distribution are used.

Thus, the multi-objective function can be rewritten as

$$
\Psi=\omega_{e} \Phi+\omega_{v} \beta_{k} J_{v}+\omega_{s} \gamma_{k} J_{s s}
$$

where energy dissipation $\Phi$ is the main term of the multi-objective function.

As follows in Chapter 6, the total shear stress and vorticity objective functions do not converge to a final discrete distribution when are evaluated individually. Satisfactory results are obtained only when the term which minimizes flow in porous media $\left(\int_{\Omega} \alpha(\rho) \mathbf{u}^{2} d \Omega\right)$ is included. Thus, these functions must combined with the total energy dissipation function or with the porous media term in order to achieve a domain distribution without intermediate values.

\subsubsection{Volume Constraint}

As a constraint, a given fraction of the design domain $V$ is allowed to be occupied by fluid and the remaining must be solid, as defined in Equation 3.13 .

$$
\int_{\Omega} \rho d \Omega \leq V
$$

This constraint is commonly used in solid mechanics field, to limit the amount of material or weight that a system can have. In the studied case, this weight limit is not 
relevant, however, it is necessary to apply this constraint in order to not obtain a final topology where the entire domain is filled with fluid, because the value of the function presented in Equation 3.5 is minimized when the values of velocity $\mathbf{u}$ are reduced, which occurs when the area occupied by fluid is augmented.

In addition, the value of the design variable must be within the range $0 \leq \rho \leq 1$, as described before, and the Navier-Stokes constitutive equations must be satisfied.

\subsection{Discrete Form}

In this section, it will be presented the discrete form of each objective function previously described.

The discrete form of the energy dissipation objective function is defined by (ROMERO; SILVA, 2014):

$$
\Phi=\frac{1}{2} \mathbf{u}^{\mathbf{T}}\left(\overline{\mathbf{K}}_{\mathbf{d}}+\overline{\mathbf{K}}_{\alpha}\right) \mathbf{u}=\frac{1}{2} \mathbf{z}^{\mathbf{T}} \mathbf{C} \mathbf{z}
$$

where the vector $\mathbf{z}=\left[\begin{array}{lll}\mathbf{u}_{\mathbf{1}} & \mathbf{u}_{\mathbf{2}} & \mathbf{p}\end{array}\right]^{T}$ and the matrix $\mathrm{C}$ are given by

$$
\begin{gathered}
C=\left[\begin{array}{cc}
\overline{\mathbf{K}}_{\mathbf{d}}+\overline{\mathbf{K}}_{\alpha} & 0 \\
0 & 0
\end{array}\right] \\
\overline{\mathbf{K}}_{\mathbf{d}}=\left[\begin{array}{cc}
2 \mathbf{K}_{\mathbf{1 1}}+\mathbf{K}_{\mathbf{2 2}} & \mathbf{K}_{\mathbf{1 2}} \\
\mathbf{K}_{\mathbf{2 1}} & \mathbf{K}_{\mathbf{1 1}}+2 \mathbf{K}_{\mathbf{2 2}}
\end{array}\right] \\
\overline{\mathbf{K}}_{\alpha}=\left[\begin{array}{cc}
\mathbf{K}_{\alpha} & 0 \\
0 & \mathbf{K}_{\alpha}
\end{array}\right]
\end{gathered}
$$

and the matrices $\mathbf{K}_{\alpha}$ and $\mathbf{K}_{i j}$ are defined in Section 2.3 .

The discrete form of the vorticity objective function is obtained by performing the numerical integration of functional presented in Equation 3.7.

$$
J(\mathbf{u})=\sum_{e=1}^{n e} \int_{\Omega^{e}}\left(\frac{\partial u_{2}}{\partial x_{1}}-\frac{\partial u_{1}}{\partial x_{2}}\right)^{2} d \Omega=\sum_{e=1}^{n e} \mathbf{u}_{\mathbf{e}}^{T} \mathbf{M}_{v, e} \mathbf{u}_{\mathbf{e}}
$$

where $n e$ is the total number of elements, the underscript $e$ indicates that the matrices are assembled per element, $\mathbf{u}=\left[u_{1} u_{2}\right]^{T} u_{1}, u_{2}$ are vectors with nodal values of velocity in the $x_{1}$ and $x_{2}$ directions, respectively, and the local matrix $\mathbf{M}_{\mathbf{v}, \mathbf{e}}$ is defined as

$$
\mathbf{M}_{v, e}=\left[\begin{array}{ll}
\mathbf{M}_{v, e}^{11} & \mathbf{M}_{v, e}^{12} \\
\mathbf{M}_{v, e}^{21} & \mathbf{M}_{v, e}^{22}
\end{array}\right]
$$


where

$$
\begin{aligned}
& \mathbf{M}_{v, e}^{11}=\int_{\Omega^{e}}\left(\frac{\partial \Psi}{\partial x_{2}} \frac{\partial \boldsymbol{\Psi}^{T}}{\partial x_{2}}\right) d \Omega ; \quad \mathbf{M}_{v, e}^{12}=-\int_{\Omega^{e}}\left(\frac{\partial \Psi}{\partial x_{2}} \frac{\partial \Psi^{T}}{\partial x_{1}}\right) d \Omega ; \\
& \mathbf{M}_{v, e}^{21}=-\int_{\Omega^{e}}\left(\frac{\partial \Psi}{\partial x_{1}} \frac{\partial \boldsymbol{\Psi}^{T}}{\partial x_{2}}\right) d \Omega ; \quad \mathbf{M}_{v, e}^{22}=\int_{\Omega^{e}}\left(\frac{\partial \boldsymbol{\Psi}}{\partial x_{1}} \frac{\partial \boldsymbol{\Psi}^{T}}{\partial x_{1}}\right) d \Omega ;
\end{aligned}
$$

and $\boldsymbol{\Psi}$ are interpolation functions for the triangular element presented in Section 2.3.2.

The discrete form of shear stress functional is expressed by:

$$
\mathbf{J}_{\mathbf{s}} \mathbf{s}=\frac{1}{2} \mathbf{u}^{\mathbf{T}}\left(\overline{\mathbf{K}}_{\mathbf{s}}\right) \mathbf{u}
$$

where the matrix $\overline{\mathbf{K}}_{\mathbf{s}}$ is defined by

$$
\overline{\mathbf{K}}_{\mathbf{s}}=\left[\begin{array}{cc}
2 \mathbf{K s}_{\mathbf{1 1}}+\mathbf{K s}_{\mathbf{2 2}} & \mathbf{K} \mathbf{s}_{\mathbf{1 2}} \\
\mathbf{K s}_{\mathbf{2 1}} & \mathbf{K s}_{\mathbf{1 1}}+2 \mathbf{K s}_{\mathbf{2 2}}
\end{array}\right]
$$

with the following matricial coefficients:

$$
\mathbf{K}_{i j}=\int_{\Omega^{e}} \frac{\partial \Psi}{\partial x_{j}} \frac{\partial \Psi^{T}}{\partial x_{i}} d x
$$




\section{SENSITIVITIES CALCULATION}

In this work, a gradient-based optimization algorithm is used, which requires the derivative of the model output with respect to its input parameters, i.e, the derivative of the functional of interest in relation to the design variable must be calculated. The sensitivities calculation is computed through the adjoint method (FUNKE, 2013).

\subsection{Adjoint Method}

In the development of this section, the following considerations are taken in to account: $F(u, m)=0$ is the forward model defined by the PDE of the governing equations, where $m$ is the design variable and $u$ is the solution vector. $J(u, m)$ is the defined functional of interest.

Thus, the calculation of the total derivative $\frac{d J}{d m}$ can be done by applying the chain rule

$$
\frac{d J}{d m}=\frac{\partial J}{\partial u} \frac{d u}{d m}+\frac{\partial J}{\partial m}
$$

The derivative of $u$ is obtained by taking the derivative of the FEM system of equations (forward model), since the PDE operator $F$ is defined in explicit form.

$$
\begin{gathered}
\frac{\partial F}{\partial u} \frac{d u}{d m}+\frac{\partial F}{\partial m}=0 \\
\frac{d u}{d m}=-\left(\frac{\partial F}{\partial u}\right)^{-1} \frac{\partial F}{\partial m}
\end{gathered}
$$

Thus, the desired derivative is given by

$$
\frac{d J}{d m}=-\frac{\partial J}{\partial u}\left(\frac{\partial F}{\partial u}\right)^{-1} \frac{\partial F}{\partial m}+\frac{\partial J}{\partial m}
$$

The adjoint vector $\lambda^{*}$ is defined as

$$
\lambda^{*}=-\frac{\partial J}{\partial u}\left(\frac{\partial F}{\partial u}\right)^{-1}
$$

where $*$ represents the complex conjugate of the transpose.

To summarize, the adjoint method first solves the adjoint equation

$$
\frac{\partial F^{*}}{\partial u} \lambda=\frac{\partial J^{*}}{\partial u}
$$


and then uses the adjoint solution to compute the functional gradient.

$$
\frac{d J}{d m}=-\lambda^{*} \frac{\partial F}{\partial m}+\frac{\partial J}{\partial m}
$$

\subsection{Energy Dissipation Sensitivity Analysis}

The gradient of the objective function $\Phi$ is calculated by using the adjoint method described in Section 4.1,

The gradient of energy dissipation with respect to the design variable $\rho_{i}$ is obtained by differentiating Equation 3.14. Notice that the matrix $\mathbf{C}$ is symmetric, thus $\mathbf{C}^{T}=\mathbf{C}$.

$$
\frac{\partial \Phi}{\partial \rho_{i}}=\frac{1}{2}\left[\left(\frac{\partial z}{\partial \rho_{i}}\right)^{T} \mathbf{C} \mathbf{z}+\mathbf{z}^{T} \frac{\partial \mathbf{C}}{\partial \rho_{i}} \mathbf{z}+\mathbf{z}^{T} \mathbf{C} \frac{\partial \mathbf{z}}{\partial \rho_{i}}\right]=\frac{1}{2} \mathbf{z}^{T} \frac{\partial \mathbf{C}}{\partial \rho_{i}} \mathbf{z}+\mathbf{z}^{T} \mathbf{C} \frac{\partial \mathbf{z}}{\partial \rho_{i}}
$$

The derivative $\frac{\partial \mathbf{z}}{\partial \rho_{i}}$ can be obtained through the FEM equilibrium equation. The residual $\mathbf{R}$ is given by:

$$
\mathbf{R}=\mathbf{K}_{G} \mathbf{z}-\mathbf{F}
$$

where

$$
\mathbf{K}_{G}=\left[\begin{array}{cc}
\mathbf{C}(\mathbf{u})+\mathbf{K} & -\mathbf{Q} \\
-\mathbf{Q}^{T} & 0
\end{array}\right]
$$

Thus, by differentiating Equation 4.9 with respect to the design variable $\rho$

$$
\frac{d \mathbf{R}}{d \rho_{i}}=\frac{\partial \mathbf{R}}{\partial \mathbf{z}} \frac{d \mathbf{z}}{d \rho_{i}}+\frac{\partial \mathbf{R}}{\partial \rho_{i}}
$$

where $\frac{\partial \mathbf{R}}{\partial \mathbf{z}}=J$ is the Jacobian matrix resulting from the application of Newton's method to the solution of the nonlinear system.

Thus,

$$
\mathbf{J} \frac{d \mathbf{z}}{d \rho_{i}}=-\frac{\partial \mathbf{R}}{\partial \rho_{i}}=-\frac{\partial \mathbf{K}_{\mathbf{g}}}{\partial \rho_{i}} \mathbf{z}+\frac{\partial \mathbf{F}}{\partial \rho_{i}}=\mathbf{r}_{\mathbf{i}}
$$

From Equation 4.12 ,

$$
\frac{d \mathbf{z}}{d \rho_{i}}=\mathbf{J}^{-1} \mathbf{r}_{\mathbf{i}}
$$

where the vector $\mathbf{r}_{i}$ is calculated for each element $i$ in the domain. Therefore, the second term of Equation 4.8 becomes

$$
\mathbf{z}^{T} \mathbf{C} \frac{\partial \mathbf{z}}{\partial \rho_{i}}=\mathbf{z}^{T} \mathbf{C} \mathbf{J}^{-1} \mathbf{r}_{i}=\mathbf{S}^{T} \mathbf{r}_{i}
$$




$$
\mathbf{S}^{T}=\mathbf{z}^{T} \mathbf{C} \mathbf{J}^{-1}
$$

The following linear system is obtained by rearranging the previous expressions

$$
\mathbf{J}^{T} \mathbf{S}=\mathbf{C z}
$$

Thus, the vector $\mathbf{S}$ is calculated by solving the above linear system. This system is independent of the derivative with respect to design variable $\rho$, so it is solved once per iteration of the optimization process by using the velocity field $\mathbf{u}$ computed in the previous iteration. Then, by replacing the vector $\mathbf{S}$ in Equation 4.15 , the unknown term $\frac{\partial \mathbf{z}}{\partial \rho_{i}}$ and thus, the energy dissipation gradient is obtained.

The sensitivity of $\frac{\partial \mathbf{C}}{\partial \rho_{i}}$ can be computed as a sum of the gradients of $\overline{\mathbf{K}}_{\alpha}$ and $\overline{\mathbf{K}}_{\mathbf{d}}$ with respect to the design variable.

$$
\frac{\partial \mathbf{C}}{\partial \rho_{i}}=\left[\begin{array}{cc}
\frac{\partial \overline{\mathbf{K}}_{\mathbf{d}}}{\partial \rho_{i}}+\frac{\partial \overline{\mathbf{K}}_{\alpha}}{\partial \rho_{i}} & 0 \\
0 & 0
\end{array}\right]
$$

These gradients are calculated by

$$
\begin{gathered}
\frac{\partial \overline{\mathbf{K}}_{\alpha}}{\partial \rho_{i}}=\frac{\partial \alpha\left(\rho_{i}\right)}{\partial \rho_{i}}\left[\begin{array}{cc}
k_{\alpha, e} & 0 \\
0 & k_{\alpha, e}
\end{array}\right] \\
\frac{\partial \overline{\mathbf{K}}_{\mathbf{d}}}{\partial \rho_{i}}=\frac{\partial \mu\left(\rho_{i}\right)}{\partial \rho_{i}}\left[\begin{array}{ll}
k_{11, e} & k_{12, e} \\
k_{21, e} & k_{22, e}
\end{array}\right]
\end{gathered}
$$

where the matrix coefficients $k_{\alpha}$ and $k_{i j}$, independent from the design variable $\rho$, are defined as:

$$
\begin{aligned}
k_{\alpha, e} & =\int_{\Omega} \boldsymbol{\Psi} \boldsymbol{\Psi}^{T} d x \\
k_{i j, e} & =\int_{\Omega} \frac{\partial \boldsymbol{\Psi}}{\partial x_{j}} \frac{\partial \boldsymbol{\Psi}^{T}}{\partial x_{i}} d x, \quad i, j=1,2 ;
\end{aligned}
$$

From Equation 3.1 it is obtained:

$$
\frac{\partial \alpha(\rho)}{\partial \rho_{i}}=\left(\alpha_{f}-\alpha_{s}\right) \frac{q(q+1)}{(q+\rho)^{2}}
$$

The term $\mu(\rho)$ is also dependant of the shear-rate. Thus, it is necessary to apply the 
chain rule to obtain the viscosity derivative:

$$
\frac{\partial \mu(\dot{\gamma})}{\partial \rho_{i}}=\frac{\partial \mu(\dot{\gamma})}{\partial \mathbf{u}} \frac{\partial \mathbf{u}}{\partial \rho_{i}}
$$

When a viscosity interpolation is applied, its derivative should also be considered. For the interpolation presented in Equations 3.2 the derivative with the respect to the design variable is:

$$
\frac{\partial \mu(\rho)}{\partial \rho_{i}}=\left(\mu_{f}(\dot{\gamma})-\mu_{s}\right) \frac{q(q+1)}{(q+\rho)^{2}}+\frac{\partial \mu_{f}(\dot{\gamma})}{\partial \rho_{i}} \frac{\rho(1+q)}{(\rho+q)}
$$

and for the interpolation present in Equation 3.3, we have

$$
\frac{\partial \mu(\rho)}{\partial \rho_{i}}=\left(\mu_{0}-\mu_{\infty}\right)\left\{-K(1-\rho)^{K-1}\left[1+\left(\lambda \dot{\gamma}^{a}\right]^{\frac{n-1}{a}}\right]+(1-\rho)^{K}(n-1)(\lambda \dot{\gamma})^{a-1}\left[1+(\lambda \dot{\gamma})^{a}\right]^{\frac{n-1-a}{a}} \frac{\partial \dot{\gamma}}{\partial \rho_{i}}\right\}
$$

where the derivative of the shear rate is calculated by

$$
\frac{\partial \dot{\gamma}}{\partial \rho_{i}}=\frac{\partial \dot{\gamma}}{\partial \mathbf{u}} \frac{\partial \mathbf{u}}{\partial \rho_{i}}
$$

\subsubsection{Sensitivity of Viscosity}

As described before, in order to obtain the gradient of the matrix $\mathbf{C}$, the derivative $\frac{\partial \mu_{f}(\dot{\gamma})}{\partial \mathbf{u}}$ must be computed.

Considering the shear rate for bi-dimensional flow, Equation 2.9 becomes:

$$
\dot{\gamma}=\left[2\left(\frac{\partial u_{1}}{\partial x_{1}}\right)^{2}+2\left(\frac{\partial u_{2}}{\partial x_{2}}\right)^{2}+\left(\frac{\partial u_{1}}{\partial x_{2}}+\frac{\partial u_{2}}{\partial x_{1}}\right)^{2}\right]^{1 / 2}
$$

The derivative of viscosity with respect to velocity is obtained by the chain rule:

$$
\frac{\partial \mu_{f}(\dot{\gamma})}{\partial \mathbf{u}}=\frac{\partial \mu_{f}(\dot{\gamma})}{\partial \dot{\gamma}} \frac{\partial \dot{\gamma}}{\partial \mathbf{u}}
$$

where the first term corresponds to the derivative of Equation 2.11

$$
\frac{\partial \mu_{f}(\dot{\gamma})}{\partial \dot{\gamma}}=(n-1) \lambda\left(\mu_{0}-\mu_{\infty}\right)\left[1+(\lambda \dot{\gamma})^{a}\right]^{\frac{n-1}{a}-1}(\lambda \dot{\gamma})^{a-1}
$$

From Equation 4.26 ,

$$
\frac{\partial \dot{\gamma}}{\partial \mathbf{u}_{\mathbf{1}}}=\dot{\gamma}^{-1}\left[2 \frac{\partial u_{1}}{\partial x_{1}} \frac{\partial \psi}{\partial x_{1}}+\left(\frac{\partial u_{1}}{\partial x_{2}}+\frac{\partial u_{2}}{\partial x_{1}}\right) \frac{\partial \psi}{\partial x_{2}}\right]
$$




$$
\frac{\partial \dot{\gamma}}{\partial \mathbf{u}_{2}}=\dot{\gamma}^{-1}\left[2 \frac{\partial u_{2}}{\partial x_{2}} \frac{\partial \psi}{\partial x_{2}}+\left(\frac{\partial u_{1}}{\partial x_{2}}+\frac{\partial u_{2}}{\partial x_{1}}\right) \frac{\partial \psi}{\partial x_{1}}\right]
$$

where $\psi$ is the velocity interpolation function defined in Section 2.3 .2

Thus, the system can be represented using the matrix form:

$$
\frac{\partial \dot{\gamma}}{\partial \mathbf{u}}=\dot{\gamma}^{-1} \mathbf{M}_{\mathbf{g}} \mathbf{u}
$$

where

$$
\mathbf{M}_{\mathbf{g}}=\left[\begin{array}{cc}
2 \frac{\partial \boldsymbol{\Psi}}{\partial x_{1}} \frac{\partial \boldsymbol{\Psi}^{T}}{\partial x_{1}}+\frac{\partial \boldsymbol{\Psi}}{\partial x_{2}} \frac{\partial \boldsymbol{\Psi}^{T}}{\partial x_{2}} & \frac{\partial \boldsymbol{\Psi}}{\partial x_{2}} \frac{\partial \boldsymbol{\Psi}^{T}}{\partial x_{1}} \\
\frac{\partial \boldsymbol{\Psi}}{\partial x_{1}} \frac{\partial \boldsymbol{\Psi}^{T}}{\partial x_{2}} & 2 \frac{\partial \boldsymbol{\Psi}}{\partial x_{2}} \frac{\partial \boldsymbol{\Psi}^{T^{T}}}{\partial x_{2}}+\frac{\partial \boldsymbol{\Psi}}{\partial x_{1}} \frac{\partial \boldsymbol{\Psi}^{T}}{\partial x_{1}}
\end{array}\right]
$$

\subsection{Vorticity Sensitivity Analysis}

The vorticity sensitivity is obtained by differentiating Equation 3.7 with respect to design variable $\rho$ (ROMERO; SILVA, 2014).

$$
\frac{d J_{v}}{d \rho_{i}}=\frac{d \mathbf{u}^{T}}{d \rho_{i}} \mathbf{M}_{v} \mathbf{u}+\mathbf{u}^{T} \frac{\partial \mathbf{M}_{v}}{\partial \rho_{i}} \mathbf{u}+\mathbf{u}^{T} \mathbf{M}_{v} \frac{d \mathbf{u}}{d \rho_{i}}
$$

Since $\mathbf{M}_{v}$ is not a direct function of the design variable $\rho, \frac{\partial \mathbf{M}_{v}}{\partial \rho_{i}}=0$, thus

$$
\frac{d J}{d \rho_{i}}=\mathbf{u}^{T}\left(\mathbf{M}_{v}+\mathbf{M}_{v}^{T}\right) \frac{d \mathbf{u}}{d \rho_{i}}
$$

The term $\frac{d \mathbf{u}}{d \rho_{i}}$ can be obtained from system FEM equations and with the use of an auxiliary matrix $\mathbf{L}^{T}$ the velocities are isolated from the vector $\mathbf{z}$

$$
\mathbf{u}=\mathbf{L}^{T} \mathbf{z} \Longrightarrow \frac{d \mathbf{u}}{\rho_{i}}=\mathbf{L}^{T} \frac{d \mathbf{z}}{\rho_{i}}
$$

where $\mathbf{L}^{T}$ is defined as:

$$
\mathbf{L}^{T}=\left[\begin{array}{ll}
\mathbf{I}_{n \times n} & \mathbf{0}_{n \times m}
\end{array}\right] .
$$

The matrix $\mathbf{L}$ is composed by identity matrix $\mathbf{I}$, of dimension $(n \times n)$, where $n$ is the number of degrees of freedom of velocity, and an array of dimension $(n \times m)$ which consists only of zeros, where $m$ is the number of pressure degrees of freedom.

From Equations 4.13 and 4.35

$$
\frac{d \mathbf{z}}{d \rho_{i}}=\mathbf{J}^{-1} \mathbf{r}_{\mathbf{i}} \Longrightarrow \frac{d \mathbf{u}}{d \rho_{i}}=\mathbf{L}^{T} \mathbf{J}^{-1} \mathbf{r}_{i}
$$


By replacing Equation 4.34 on Equation 4.37, we obtain:

$$
\frac{d J}{d \rho_{i}}=\mathbf{u}^{T}\left(\mathbf{M}_{v}+\mathbf{M}_{v}^{T}\right) \mathbf{L}^{T} \mathbf{J}^{-1} \mathbf{r}_{i}=\mathbf{S}_{v}^{T} \mathbf{r}_{i}
$$

where,

$$
\mathbf{S}_{v}^{T}=\mathbf{u}^{T}\left(\mathbf{M}_{v}+\mathbf{M}_{v}^{T}\right) \mathbf{L}^{T} \mathbf{J}^{-1}
$$

By rearranging the previous expression, we obtain the linear system

$$
\mathbf{J}^{T} \mathbf{S}_{v}=\mathbf{L}\left(\mathbf{M}_{v}+\mathbf{M}_{v}^{T}\right) \mathbf{u}
$$

The $\mathbf{S}_{v}$ vector is obtained by solving this linear system. This system is solved once per iteration of the optimization process, since it is independent of the derivative with respect to the design variable. It depends on the previous velocity field $\mathbf{u}$ and the residual $R$ is assumed to be zero. The vector $r_{i}$, from Equations 4.38, given by Equation 4.12, is calculated for each element $i$ within the domain $\Omega$.

\subsection{Shear Stress Sensitivity Analysis}

The gradients of shear stress objective function with respect to the fluid pseudo-density $\rho$ is obtained by using the same method as described before.

$$
\frac{d \mathbf{J}_{\mathbf{s s}}}{d \rho_{i}}=\frac{1}{2}\left[\left(\frac{\partial \mathbf{u}}{\partial \rho_{i}}\right)^{T} \overline{\mathbf{K}}_{\mathbf{s}} \mathbf{u}+\mathbf{u}^{T} \frac{\partial \overline{\mathbf{K}}_{\mathbf{s}}}{\partial \rho_{i}} \mathbf{u}+\mathbf{u}^{T} \overline{\mathbf{K}}_{\mathbf{s}} \frac{\partial \mathbf{u}}{\partial \rho_{i}}\right]
$$

Since the matrix $\overline{\mathbf{K}}_{\mathbf{s}}$ is independent of the design variable, $\frac{\partial \overline{\mathbf{K}}_{\mathbf{s}}}{\partial \rho_{i}}=0$ and thus

$$
\frac{d \mathbf{J}_{\mathbf{s s}}}{d \rho_{i}}=\mathbf{u}^{T}\left(\overline{\mathbf{K}}_{\mathbf{s}}+\overline{\mathbf{K}}_{\mathbf{s}}^{T}\right) \frac{d \mathbf{u}}{d \rho_{i}}
$$

The derivative $\frac{d \mathbf{u}}{d \rho_{i}}$ can be obtained as described before in Equation 4.37

$$
\frac{d \mathbf{J}_{\mathbf{s s}}}{d \rho_{i}}=\mathbf{u}^{T}\left(\overline{\mathbf{K}}_{\mathbf{s}}+\overline{\mathbf{K}}_{\mathbf{s}}^{T}\right) \mathbf{L}^{T} \mathbf{J}^{-1} \mathbf{r}_{i}=\mathbf{S}_{s}^{T} \mathbf{r}_{i}
$$

The considerations for the system above and the vectors $\mathbf{S}_{\mathbf{t}}$ and $\mathbf{r}_{\mathbf{i}}$ are the same as stated for the energy dissipation and vorticity objective functions. 


\subsection{Sensitivity Analysis of Multi-Objective Function}

The gradient of multi-objective function (Eq. 3.12 is given by:

$$
\frac{d \psi}{d \rho_{i}}=\omega_{e} \frac{d \mathbf{\Phi}}{d \rho_{i}}+\omega_{v} \beta_{k} \frac{d \mathbf{J}_{\mathbf{v}}}{d \rho_{i}}+\omega_{v} \frac{\partial \beta_{k}}{\partial \rho_{i}} d \mathbf{J}_{\mathbf{v}}+\omega_{s s} \gamma_{k} \frac{d \mathbf{J}_{\mathbf{s s}}}{d \rho_{i}}+\omega_{s s} \frac{\partial \gamma_{k}}{\partial \rho_{i}} d \mathbf{J}_{\mathbf{s s}}
$$

The sensitivities of $\beta_{k}$ and $\gamma_{k}$ are terms that arise due to the derived chain rule. Since $\beta_{k}$ and $\gamma_{k}$ represent constant values within each iteration, the derivative of these terms with respect to design variable $\rho$ is null. Thus, (ZHU; ZHANG; FATIKOW, 2014):

$$
\begin{aligned}
& \frac{\partial \beta_{k}}{\partial \rho_{i}}=0 \\
& \frac{\partial \gamma_{k}}{\partial \rho_{i}}=0
\end{aligned}
$$

Thus, we can define a simplified gradient of the multi-objective function for each optimization iteration as:

$$
\frac{d \psi}{d \rho_{i}}=\omega_{e} \frac{d \mathbf{\Phi}}{d \rho_{i}}+\omega_{v} \frac{d \mathbf{J}_{\mathbf{v}}}{d \rho_{i}}+\omega_{s s} \frac{d \mathbf{J}_{\mathbf{s s}}}{d \rho_{i}}
$$

The terms $\frac{d \mathbf{\Phi}}{d \rho_{i}}, \frac{d \mathbf{J}_{\mathbf{v}}}{d \rho_{i}}$ and $\frac{d \mathbf{J}_{\mathbf{s s}}}{d \rho_{i}}$, which correspond to energy, vorticity and shear stress sensitivities, respectively, have been calculated previously.

The energy dissipation sensitivity is

$$
\frac{\partial \Phi}{\partial \rho_{i}}=\frac{1}{2} \mathbf{z}^{T} \frac{\partial \mathbf{C}}{\partial \rho_{i}} \mathbf{z}+\mathbf{z}^{T} \mathbf{C} \mathbf{J}^{-} \mathbf{1} \mathbf{r}_{i}
$$

The vorticity sensitivity term is

$$
\frac{d J}{d \rho_{i}}=\mathbf{u}^{T}\left(\mathbf{M}_{v}+\mathbf{M}_{v}^{T}\right) \mathbf{L}^{T} \mathbf{J}^{-1} \mathbf{r}_{i}
$$

And the shear stress sensitivity contribution is

$$
\frac{d J}{d \rho_{i}}=\mathbf{u}^{T}\left(\overline{\mathbf{K}}_{\mathbf{s}}+\overline{\mathbf{K}}_{\mathbf{s}}^{T}\right) \mathbf{L}^{T} \mathbf{J}^{-1} \mathbf{r}_{i}
$$

In order to obtain the multi-objective sensitivity, the equations above can be calculated separately and its results replaced in Eq. 4.47. However, it is also possible to combine the three adjoint problems in a way that only one final adjoint equation related to the multi-objective function needs to be solved. Thus, by replacing the adjoint equations above 
in Eq. 4.47,

$$
\frac{d \Psi}{d \rho_{i}}=\omega_{e}\left[\frac{1}{2} \mathbf{z}^{T} \frac{\partial \mathbf{C}}{\partial \rho_{i}} \mathbf{z}+\mathbf{z}^{T} \mathbf{C} \mathbf{J} \mathbf{r}_{i}\right]+\omega_{v} \beta_{k} \mathbf{u}^{T}\left(\mathbf{M}_{v}+\mathbf{M}_{v}^{T}\right) \mathbf{L}^{T} \mathbf{J}^{-1} \mathbf{r}_{i}+\omega_{s} \gamma_{k} \mathbf{u}^{T}\left(\overline{\mathbf{K}}_{\mathbf{s}}+\overline{\mathbf{K}}_{\mathbf{s}}^{T}\right) \mathbf{L}^{T} \mathbf{J}^{-1} \mathbf{r}_{i}
$$

By packing the terms, it is obtained

$$
\frac{d \Psi}{d \rho_{i}}=\omega_{e} \frac{1}{2} \mathbf{z}^{T} \frac{\partial \mathbf{C}}{\partial \rho_{i}} \mathbf{z}+\left[\omega_{e} \mathbf{z}^{T} \mathbf{C}+\omega_{v} \beta_{k} \mathbf{u}^{T}\left(\mathbf{M}_{v}+\mathbf{M}_{v}^{T}\right) \mathbf{L}^{T}+\omega_{s} \gamma_{k} \mathbf{u}^{T}\left(\overline{\mathbf{K}}_{\mathbf{s}}+\overline{\mathbf{K}}_{\mathbf{s}}^{T}\right) \mathbf{L}^{T}\right] \mathbf{J}^{-1} \mathbf{r}_{i}
$$

In a simplified notation:

$$
\frac{d \Psi}{d \rho_{i}}=\omega_{e} \frac{1}{2} \mathbf{z}^{T} \frac{\partial \mathbf{C}}{\partial \rho_{i}} \mathbf{z}+\mathbf{S}_{\mathbf{m}} \mathbf{J}^{-1} \mathbf{r}_{i}
$$

where $\mathbf{S}_{\mathbf{m}}$ is obtained from the solution of the system:

$$
\mathbf{J}^{T} \mathbf{S}_{\mathbf{m}}=\left[\omega_{e} \mathbf{C z}+\omega_{v} \beta_{k} \mathbf{L}\left(\mathbf{M}_{v}+\mathbf{M}_{v}^{T}\right) \mathbf{u}+\omega_{s} \gamma_{k} \mathbf{L}\left(\overline{\mathbf{K}}_{\mathbf{s}}+\overline{\mathbf{K}}_{\mathbf{s}}^{T}\right) \mathbf{u}\right]
$$

In Equation 4.53, the vector $\mathbf{r}_{\mathbf{i}}$, given by Equation 4.12, is calculated for each element $i$ in the domain, and $\mathbf{J}$, is the Jacobian matrix resulting from the application of Newton's method to the solution of nonlinear system. 


\section{NUMERICAL IMPLEMENTATION}

\subsection{Optimization Algorithm}

In order to determine the distribution of fluid and solid material within a design domain, finite element method and an optimization algorithm are combined in an iterative process, described bellow. In this work, a gradient based algorithm is used, where the gradient information with respect to the desired functional is evaluated and gives the direction to update the design variables values.

First, the initial system information (design domain, boundaries) is defined and then the Navier-Stokes equations are solved by using finite element method. The value of the objective function and constraints are calculated with respect to the current distribution of $\rho$ and $\mathbf{u}$. Then, adjoint equations are solved in order to calculate the functional sensitivities that will be used by an optimizer to update the design variable.

This iterative process is repeated until convergence is reached, resulting in the final material distribution. Figure 5.1 presents the simplified flow chart for the implementation of the topology optimization method.

Figure 5.1 - Topology optimization implementation flow chart.

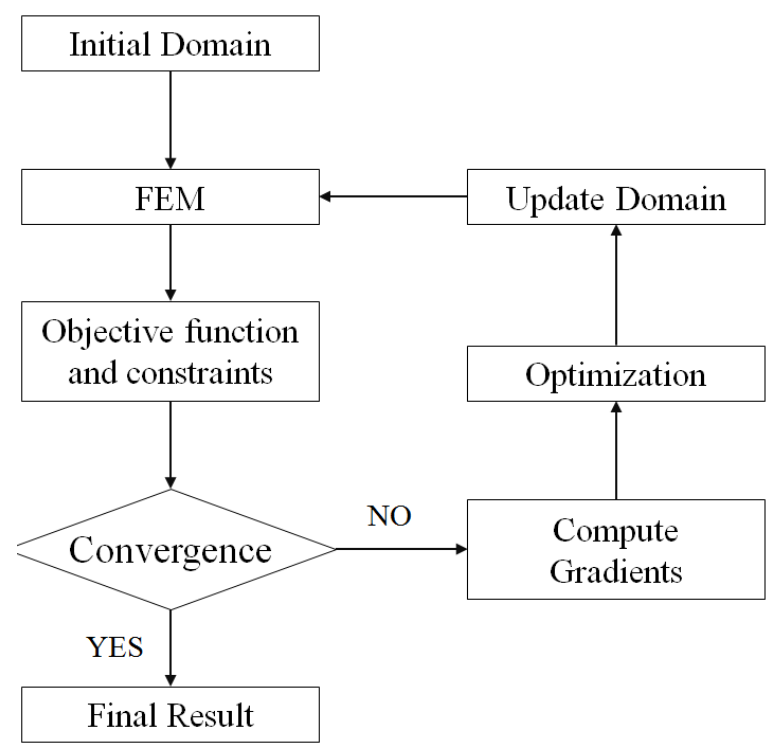

The stopping criteria for the iterative process described is given by

$$
\left\|\rho^{k}-\rho^{k-1}\right\|_{\infty} \ll 1 \times 10^{-3}
$$

where $\rho$ is the design variable distribution and $k$ is the iteration number. 


\subsection{Software Environment}

To implement the optimization software, the OpenSource FEniCS environment combined with Dolfin-Adjoint library and IPOpt optimizer package will be used. This software collection allows the automation of solving mathematical models based on partial differential equations by using Finite Element Method, through an interface written in high level code, where the PDE is described in the weak formulation.

Thus, it is possible to focus on the development and test of different formulations to the proposed problem, instead of spending time coding ordinary tasks related to most numerical simulation software, as assembly of FEM matrices and system equation solvers.

This section will give a more detailed description of how this packages and libraries are used within the topology optimization procedure.

\subsubsection{Finite Element Method}

The finite element method is implemented by using the OpenSource FEniCS environment (ALNæS et al., 2015), which consists of a collection of software components for automating the solution of PDEs. It has modules that receive the problem weak formulation in a mathematical syntax and assemble the FEM problem in a language that can be interpreted by the computer.

In listing 5.1, it is exemplified how the FEniCS coding (this case in Python) is similar to a mathematical formulation.

Listing 5.1 - FEM coding using FEniCS/Dolfin-Adjoint notation..

$$
\begin{aligned}
\mathrm{I} & =\operatorname{Identity}\left(\mathrm{U} \cdot \operatorname{cell}() \cdot \operatorname{topological} \_\mathrm{dimension}()\right) \\
\mathrm{T} & =-\mathrm{pI}+2.0 * \operatorname{visc}(\operatorname{rho}, \mathrm{u}) *(\operatorname{grad}(\mathrm{u})+\operatorname{grad}(\mathrm{u}) . \mathrm{T}) \text { \#stress tensor } \\
\mathrm{F} & =\operatorname{dens} * \operatorname{inner}(\operatorname{dot}(\operatorname{grad}(\mathrm{u}), \mathrm{u}), \mathrm{v}) * \mathrm{dx} \backslash \\
& +\operatorname{alpha}(\operatorname{rho}) * \operatorname{inner}(\mathrm{u}, \mathrm{v}) * \mathrm{dx} \backslash \\
& +\operatorname{inner}(\mathrm{T}, \operatorname{grad}(\mathrm{v})) * \mathrm{dx} \backslash \\
& -\mathrm{q} * \operatorname{div}(\mathrm{u}) * \mathrm{dx}
\end{aligned}
$$

The FEniCS main feature relies on a form compiler for compilation of code for individual forms. A Form Compiler is a type of software that can interpret equations written in a high-level language and generate a numerical routine to solve them. The FEniCS Form Compiler (FFC) is responsible for interpreting an input equation described in weak variational form through UFL (Unified Form Language) and generating an optimized low level code $(\mathrm{C}++)$ of finite element assembly in UFC (Unified Form-Assembly Code). The UFC code is combined with mesh and coefficient data, in order to assemble the FEM tensors. 
The communication between all FEniCS modules is handled by Dolfin LOGG et al. 2012), a library coded in C++/Python, which works as the main user interface of FEniCS. Dolfin is organized as a collection of FEniCS components and external software modules, each one covering a certain area of functionality, such as: linear algebra, meshes, finite elements, functions and solvers.

Some of the most important functionalities from external software are provided by the linear algebra libraries PETSc, Trilinos, uBLAS and MTL4 and the mesh partitioning libraries ParMETIS and SCOTCH.

The Finite Element equations lead to large sparse system which is non-linear and non-symmetrical, thus, an iterative solver, a more complex method than the basic Newton method is needed to guarantee the solution convergence. The FEniCS system offers pre-installed support methods such as Generalized minimal residual (Gmres) and Biconjugate gradient stabilized (Bicstab), that are also commonly used on Navier-Stokes problems. In this work, the Multifrontal Massively Parallel Sparse direct Solver (MUMPS) (MUMPS Guide, 2011) and a preconditioner that is the combination of the Incomplete LU factorization (ILU) and Block Jacobi iteration (Bjacobi) have been adopted.

\subsubsection{Sensitivities Computation}

Since it is used a gradient-based optimizer, it is necessary to derive the functional of interest in relation to design variable $\rho$. The sensitivities calculation is performed through the adjoint method, as defined in Section 4.

The implementation of the adjoint method can be based in one of the three steps needed to solve a Finite Element problem, as shown in Figure 5.2. the continuous FEM equations, the discrete FEM equations or the FEM implemented code. The resulting adjoint system will be at the same discretization stage as the model from which it is was derived. That is, the continuous adjoint equations are continuous PDE, the discrete adjoint equation are discrete PDE and the adjoint of the source code is an implementation of these discrete PDE. 
Figure 5.2 - Approaches of computing the adjoint problem.

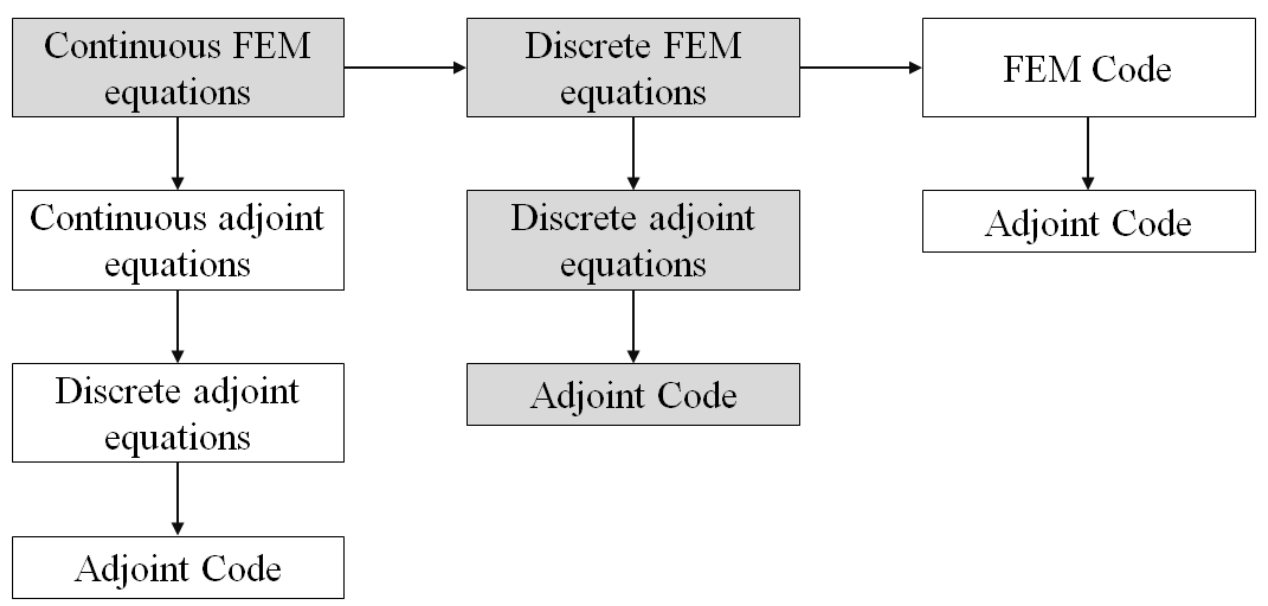

adapted from Funke (2013).

The continuous adjoint equations are obtained by deriving the adjoint system prior to the discretization of the PDE. This implies that the discontinuities that arrive from numerical methods can be avoided. Obtaining the continuous adjoint system requires a complex implementation due to manual derivation which increases considerably the development work.

It is possible to compute the derivatives with algorithmic differentiation at the last stage of the FEM implementation. This approach is performed at the lower level, i.e., the source code. The applicability can be limited and it also requires extensive user intervention and expertise.

The implemented method, illustrated as the highlighted path in Figure 5.2, consists in the adjoint derivation of the discrete equations. The main advantages obtained are that this derivation is performed independently of implementation details and the resulting gradient information is consistent with discrete finite element model.

The software library libadjoint (FUNKE, 2013) receives a high-level representation of the FEM equations and derives high-level representation of the adjoint model. It is responsible for assembling and solving the adjoint equations. The interface with FEniCS software platform is managed by the Dolfin-Adjoint package.

\subsubsection{Optimizer}

The optimization algorithm used in this work is implemented through the optimizer IPOpt, which stands for Interior Point Optimizer (WÄCHTER; BIEGLER, 2006). It is an open source software package for large-scale nonlinear optimization, written in Fortran 77 and compatible with FEniCS and Python language.

The implemented algorithm uses a primal-dual barrier method with a filter line-search 
method to solve nonlinear optimization problems which will be assumed to be stated in the generic form:

$$
\begin{aligned}
\text { Minimize : } & f(x) \\
x \in \mathbb{R}^{n} & \\
\text { subjected to: } & c(x)=0 \\
& x \geq 0
\end{aligned}
$$

In order to avoid the problem of identifying the active bound constraints, the problem constraints are replaced by a logarithmic barrier term which is added to the objective function, resulting in the following barrier problem:

$$
\begin{aligned}
\text { Minimize }: & \varphi_{\mu}(x)=f(x)-\mu \sum_{i=1}^{n} \ln \left(x^{i}\right) \\
x \in \mathbb{R}^{n} & \\
\text { subjected to: } & c(x)=0
\end{aligned}
$$

where $\varphi_{\mu}(x)$ is the barrier function, $\mu>0$ is the barrier parameter which determines the degree of influence of the barrier term $\mu \sum_{i=1}^{n} \ln \left(x^{i}\right)$.

The global convergence of the optimization is enforced by using filter line search approaches, in which the basic concept is to accept trial points that improve the objective function or improve the constraint violation instead of a combination of those two measures defined by a merit function. Thus, the barrier problem can be interpreted as a bi-objective problem with the two goals of minimizing the objective function $\varphi_{\mu_{j}}(x)$ and the constraint violation $\theta(x)=\|c(x)\|$.

A strategy for solving the original problem is to compute the solutions for a sequence of barrier problems with a decrease sequence of barrier parameters $\mu_{k}$ converging to zero, where the solution of the next barrier problem continues from the approximate solution of the previous one. The algorithm ends when the error for the barrier problem $\mu_{k+1}-\mu_{k}$ satisfies a certain specified tolerance. 


\section{NUMERICAL RESULTS}

In this chapter, numerical examples are presented to illustrate the proposed methods for non-Newtonian fluid finite element modeling and topology optimization. Blood properties have been adopted, where mass density is $\varrho=1056 \mathrm{~kg} / \mathrm{m}^{3}$ and the viscosity parameters for the Carreau-Yasuda model, defined in Section 2.2, are shown in Table 6.1 (ABRAHAM; BEHR; HEINKENSCHLOSS, 2005).

Table 6.1 - Carreau-Yasuda parameters for blood.

\begin{tabular}{cc}
\hline Parameter & Value \\
\hline$a$ & 0.64 \\
$n$ & 0.2128 \\
$\lambda$ & 8.2 \\
$\mu_{\infty}$ & 0.0035 Pa.s \\
$\mu_{0}$ & $\mu_{0}=0.16$ Pa.s \\
\hline
\end{tabular}

Firstly, the finite element method for non-Newtonian fluids is validated with a backward facing step problem. Then, optimized topologies for bi-dimensional channels are presented by comparing the constant viscosity model with shear-thinning model. Then, arterial bypass graft results are shown, for cases where the artery is fully or partially blocked. Finally, the method is extended to a tri-dimensional problem, and a 3D diffuser is presented.

\subsection{FEM Verification}

The fluid flow over a two-dimensional backward-facing step is analyzed to verify the non-Newtonian fluid model implemented. The results obtained are compared with the work of Siebert and Fodor (2009), in which a case study for different blood viscosity models in commercial software COMSOL Multiphysics is presented.

The investigated problem consists of fluid flow inside a bi-dimensional $10 \mathrm{~cm}$ long channel with a backward-facing step of $2 \mathrm{~cm}$ length and $1 \mathrm{~mm}$ height. The considered boundary conditions are presented in Figure 6.1 no-slip conditions at the top and bottom walls, a parabolic velocity profile at the inlet, with maximum velocity $U_{\max }=0.025 \mathrm{~m} / \mathrm{s}$ for the first case and $U_{\max }=0.8 \mathrm{~m} / \mathrm{s}$ for the second case, and zero pressure at the outlet. 
Figure 6.1 - Backward facing step problem.

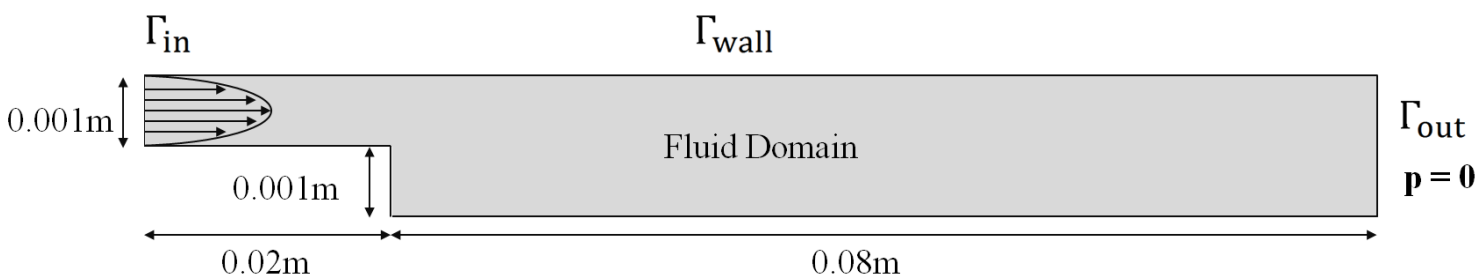

In Siebert and Fodor (2009), the Power-law, Carreau and Newtonian viscosity models are studied. In this work, Newtonian and Carreau-Yasuda models are used for the optimization problems. Even though the literature reference uses the Carreau model, for this finite element verification, the Carreau-Yasuda and Newtonian models are implemented and compared with this reference. The comparison is still valid since the Carreau model presents the same constitutive equation as the Carreau-Yasuda, however with different constants and thus, having a very similar shear-thinning behavior.

The velocity profiles for the newtonian model and two different velocities are shown in Fig. 6.2. The velocity profiles for the Carreau-Yasuda model are shown in Fig. 6.3. The influence of inlet maximum velocity can be noted in both figures. We observe that in the high velocity case a vortex arises near the backward step. Also, the maximum velocity decays faster in the low velocity case, and the flow profile becomes developed very close to the step.

Figure 6.2 - Velocity profile for newtonian model. (a) $U_{\max }=0.025 \mathrm{~m} / \mathrm{s}$. (b) $U_{\max }=0.8 \mathrm{~m} / \mathrm{s}$.

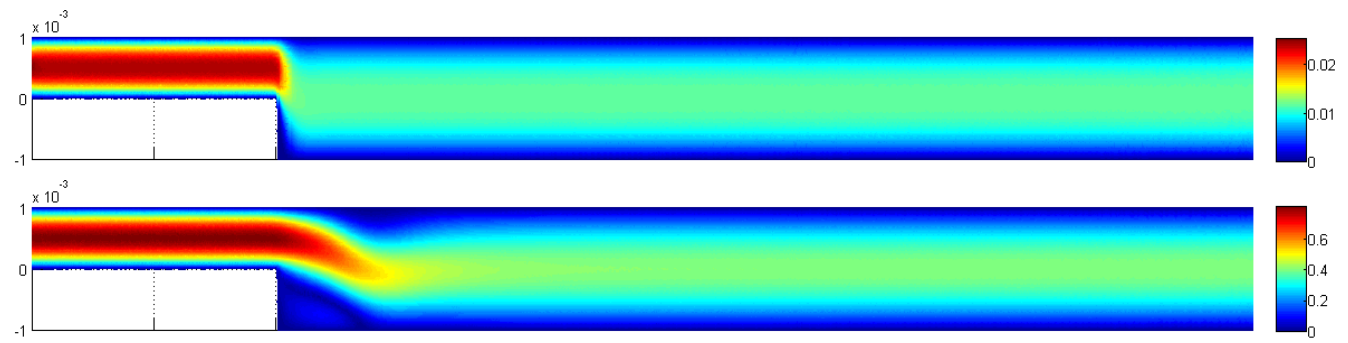

Figure 6.3 - Velocity profile for Carreau-Yasuda model. (a) $U_{\max }=0.025 \mathrm{~m} / \mathrm{s}$. (b) $U_{\max }=$ $0.8 \mathrm{~m} / \mathrm{s}$.

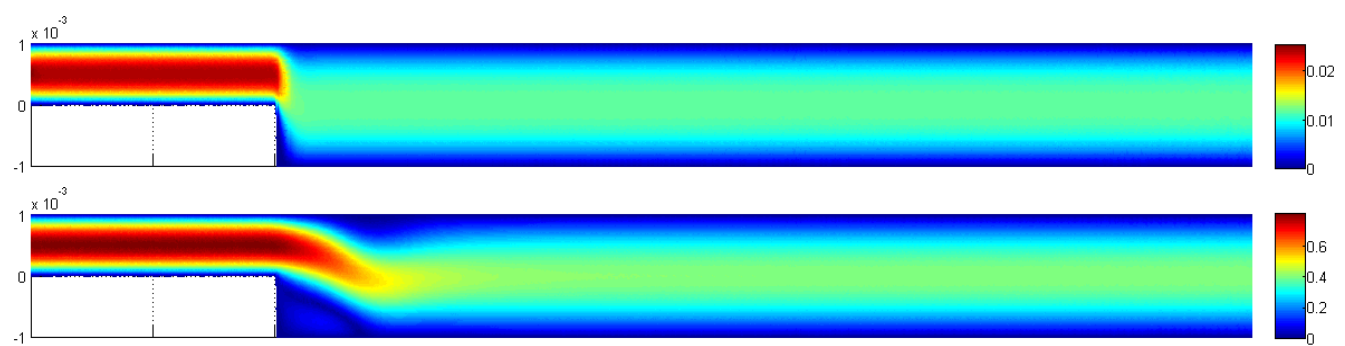

These velocity profiles for the newtonian and non-newtonian are very similar however, 
they are consistent with previous works (SIEBERT; FODOR, 2009). Taking a closer look at the profiles we can observe some differences, as shown in Fig. 6.5, in which a section of the velocity profile is taken at the outlet for each case.

Figure 6.5 shows a comparison of the velocity profiles at the outlet, which, again, correspond to the profiles obtained by Siebert and Fodor (2009) (Fig.6.4). It can be observed that for a lower inlet velocity, the non-Newtonian profiles are flatter at the center and exhibit larger velocity gradients towards the walls. Also, for higher speeds, the shear-thinning dynamic viscosity converges to Newtonian value, which leads to almost identical outlet profiles.

Even though the viscosity model adopted in this work is the Carreau-Yasuda, the results are very similar to the Carreau model and presented the same shear-thinning characteristics.

Figure 6.4 - Velocity profile obtained in literature: (a) $U_{\max }=0.025 \mathrm{~m} / \mathrm{s}$ and (b) $U_{\max }=$ $0.8 \mathrm{~m} / \mathrm{s}$.
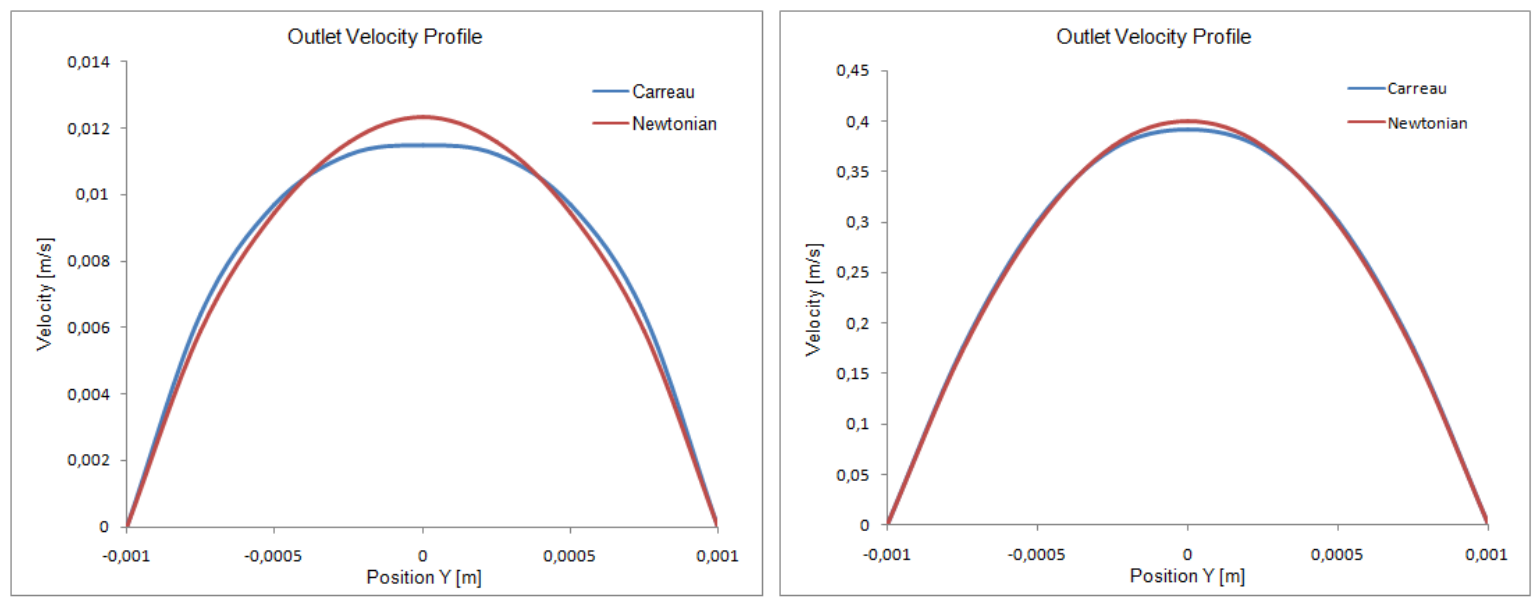

Adapted from Siebert and Fodor (2009) by interpolating the points.

Figure 6.5 - Velocity profile at the outlet for different viscosity models obtained with FEniCS: (a) $U_{\max }=0.025 \mathrm{~m} / \mathrm{s}$ and (b) $U_{\max }=0.8 \mathrm{~m} / \mathrm{s}$.
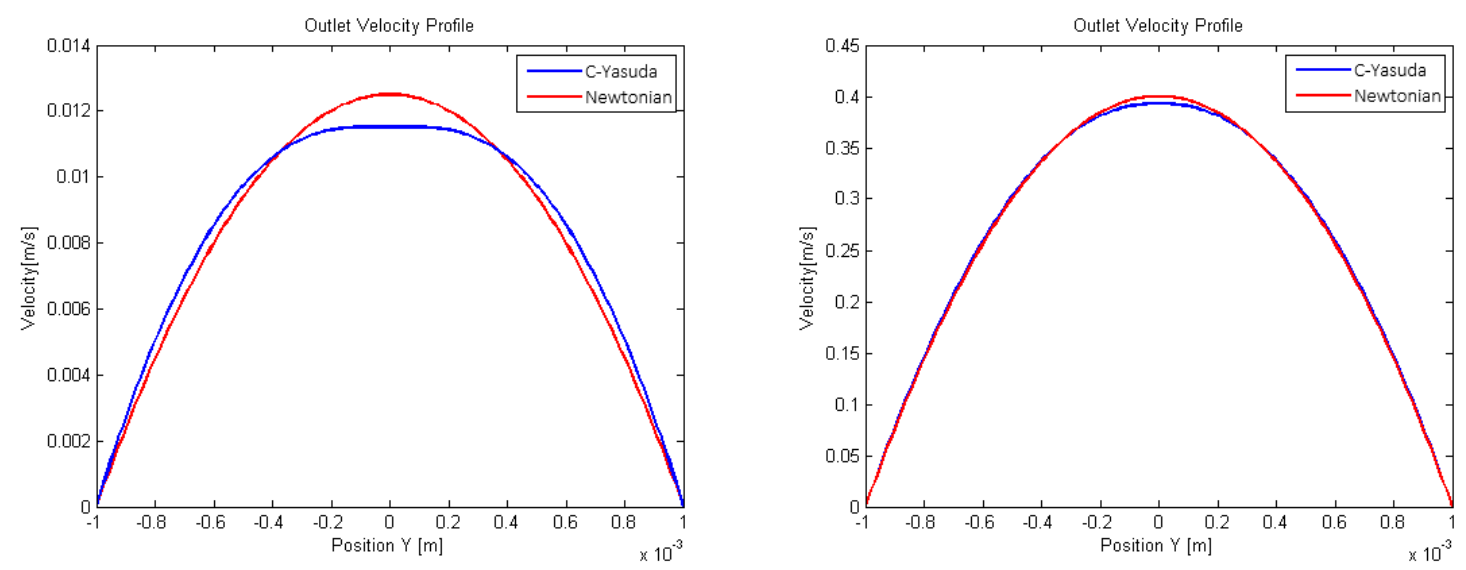


\subsection{Topology Optimization Results}

In this section, it will be presented the results obtained by applying the method described in Chapter 3 to design of blood flow channels, where the fluid is modeled as Newtonian and Non-Newtonian with Carreau-Yasuda viscosity (Eq. 2.11).

\subsubsection{Double Channel}

The double channel problem is the classical example of topology optimization applied to fluid flow. First, the results will be compared with the Stokes formulation and fictional properties $(\varrho=1)$, as studied by Borrvall and Petersson (2003). Then, optimization considering blood flow will be studied. The viscosity is modelled as constant Newtonian fluid with viscosity $\mu=1$. The objective function adopted aims to minimize the energy dissipation (Equation 3.5).

Although in the example presented in Section 6.1 the non-Newtonian velocity profile obtained presents a more flattened distribution at the center, in this case, a parabolic velocity profile is adopted in order to compare the results for different viscosity models and also confront them with the existing literature. The boundary conditions and design domain for the optimization problem are presented in Figure 6.6.

Figure 6.6 - Double channel problem.

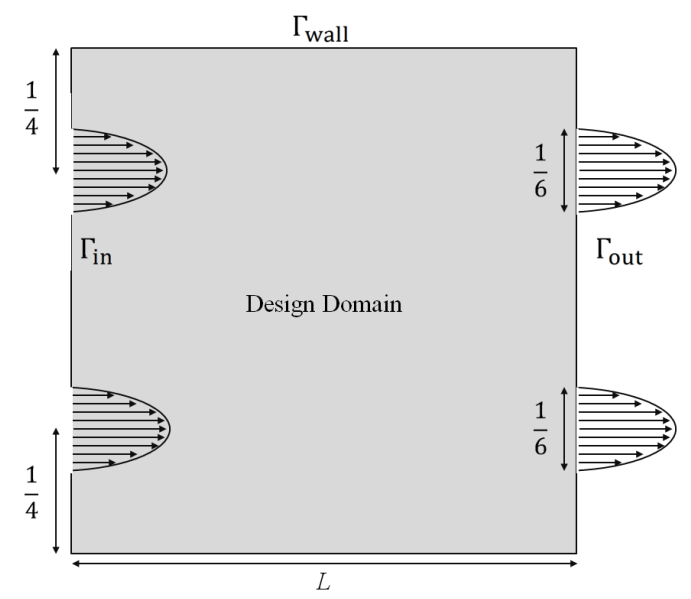

The volume constraint imposed limits in the total amount of fluid to $1 / 3$ of the domain. The adopted values for inverse permeability, as defined in Equation 3.1, are: $\alpha_{f}=0$ and $\alpha_{s}=10000$.

The height is maintained fixed while the length $L$ is variable to observe how the topology may change. Given that, the results obtained in Borrvall and Petersson (2003), for $L=1.0$ are two separated straight channels connecting the inlets to the outlets and for $L=1.5$, the channels merge into one at the center with an angle of $90^{\circ}$ between the two inlets and the two outlets, which corresponds to the analytical results obtained in 
Pironneau (1974).

Figure 6.7 shows the results obtained by using the energy functional, Stokes formulation and the Newtonian model with arbitrary properties (density $\varrho=1$ and viscosity $\mu=1$ ). We observe that the topologies are very consistent with the topologies of Borrvall and Petersson (2003), indicating that the optimization process is correctly implemented.

Figure 6.7 - Topologies for the double channel problem with Stokes formulation.

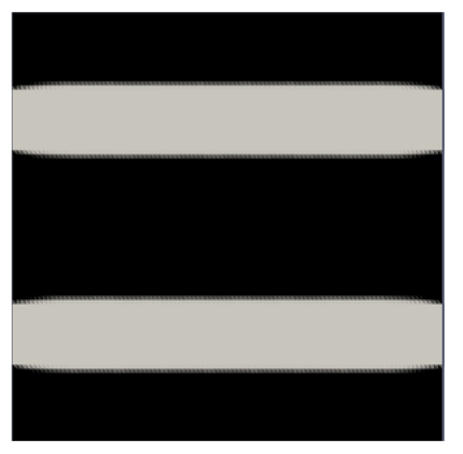

(a) $\mathrm{L}=1.0$

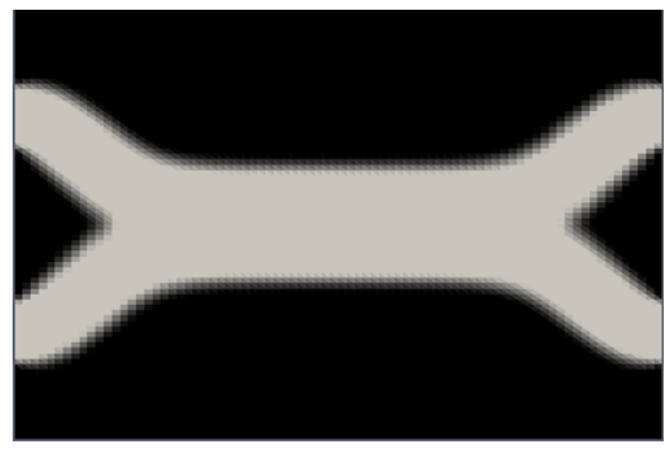

(b) $\mathrm{L}=1.5$

In addition, topology optimization for a double channel problem considering two inlets and two outlets is studied, now considering the zero stress boundary condition (Eq.2.2 equals to zero) at the outlet, as shown in Fig. 6.8. Also, the Navier-Stokes formulation of fluid flow and real blood properties are considered, as studied in Pingen and Maute (2010). Thus, the domain has dimensions in $\mathrm{cm}$, i.e., $1 \mathrm{~cm} \times 1.5 \mathrm{~cm}$. For this case, the adopted objective function aims to minimize total shear stress combined with the term $\left(\int_{\Omega} \alpha(\rho) \mathbf{u}^{2} d \Omega\right)$, necessary in order to achieve a final discrete distribution.

The optimized topologies for both Newtonian and Carreau-Yasuda (with interpolation dependant of pseudo-density) models of viscosity are presented for two different values of the maximum value of the parabolic velocity profile at inlet.

Figure 6.8 - Double channel problem with zero stress boundary condition at the outlet.

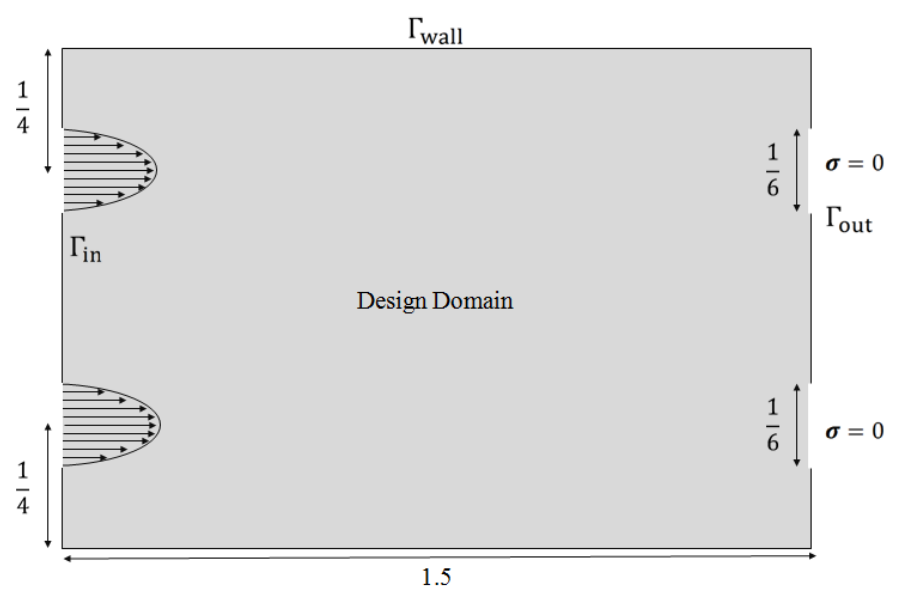


Figure 6.9 shows the results for the Newtonian model with inlet peak velocities of 0.02 and $0.2 \mathrm{~cm} / \mathrm{s}$, viscosity of $0.0035 \mathrm{~Pa} \cdot \mathrm{s}$ and considering the shear stress functional. We observe that both topologies are very similar for this model of constant viscosity. Figure 6.10 shows the velocity fields for this case. The objective function values are shown in Table 6.2.

As can be seen in Fig. 6.9, the topology for high velocity (Fig.6.9b) unite the channel farther from the inlet with a small curvature, while the topology for low velocity (Fig 6.9a) has a more straight path. This can be explained by the fact that the fluid at higher velocities has more inertia and then a smoother transition to the united channel provides a lower shear stress.

Figure 6.9 - Topology results for double channel and Navier-Stokes Newtonian model.

(a) $\operatorname{Re}=0.1$.

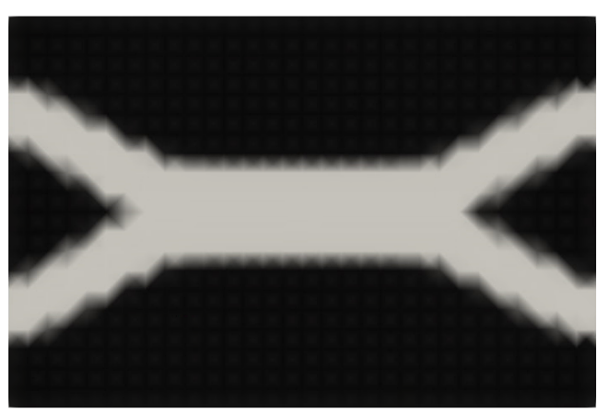

(b) $\operatorname{Re}=1.0$.

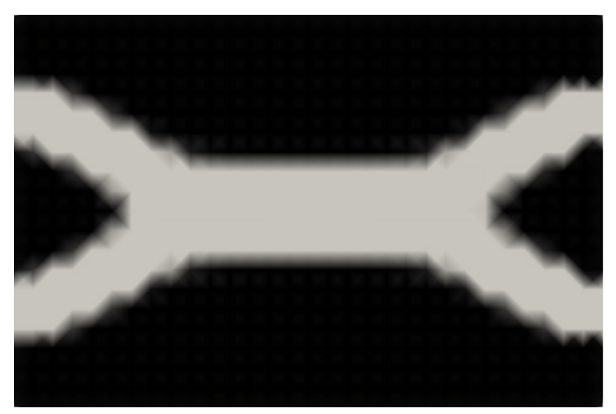

Figure 6.10 - Velocity fields for double channel and Navier-Stokes Newtonian model.

(a) $\operatorname{Re}=0.1$.

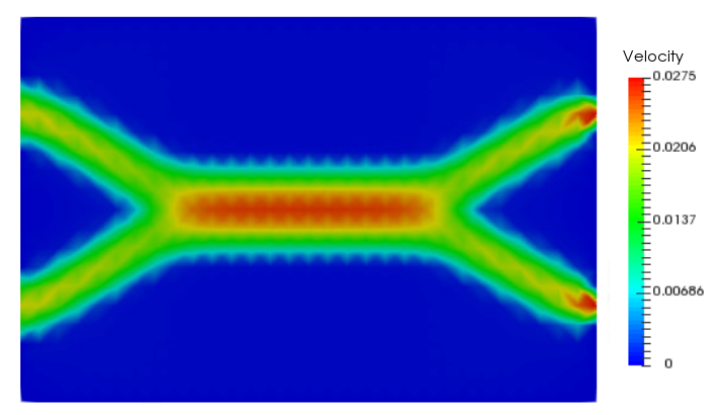

(b) $\operatorname{Re}=1.0$.

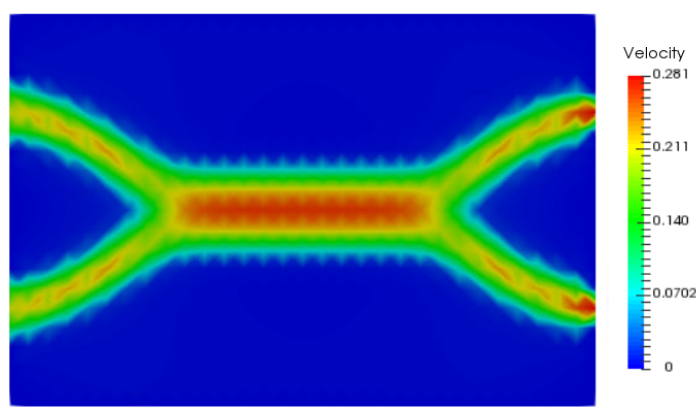

Table 6.2 - Functional values for double channel.

\begin{tabular}{lccc}
\hline Case & Inlet Velocity $[\mathrm{cm} / \mathrm{s}]$ & Area $\left[\mathrm{cm}^{2}\right]$ & Total Shear Stress \\
\hline Newtonian & 0.02 & 0.5 & 0.002 \\
Newtonian & 0.2 & 0.5 & 0.221 \\
Non Newtonian & 0.02 & 0.5 & 0.220 \\
Non Newtonian & 0.2 & 0.5 & 0.303 \\
\hline
\end{tabular}

Figure 6.11 show the results for the Non-Newtonian Carreau-Yasuda model with inlet 
peak velocities of 0.02 and $0.2 \mathrm{~cm} / \mathrm{s}$ and considering the shear stress functional. In this case, the results are very different, given that for low velocities (Fig.6.11a) the channels do not merge, while for high velocities (Fig 6.11b) the channels unite giving a topology similar to the result from the Newtonian model (Fig.6.9b). Figure 6.12 and 6.13 show the velocity and viscosity fields, respectively. The objective function values are shown in Table 6.2 .

Figure 6.11 - Topology results for double channel and Navier-Stokes non-Newtonian model (Carreau-Yasuda viscosity model).

(a) $\operatorname{Re}=0.1$.

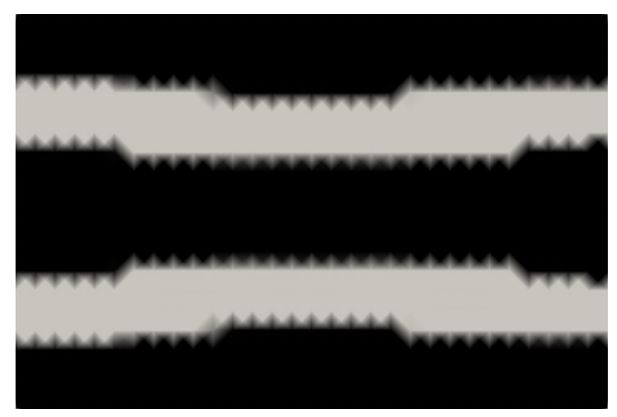

(b) $\operatorname{Re}=1.0$.

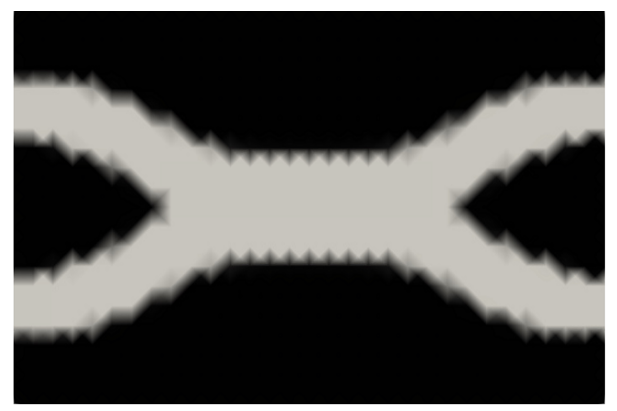

Figure 6.12 - Velocity fields for double channel and Navier-Stokes non-Newtonian model (Carreau-Yasuda viscosity model).

(a) $\operatorname{Re}=0.1$.

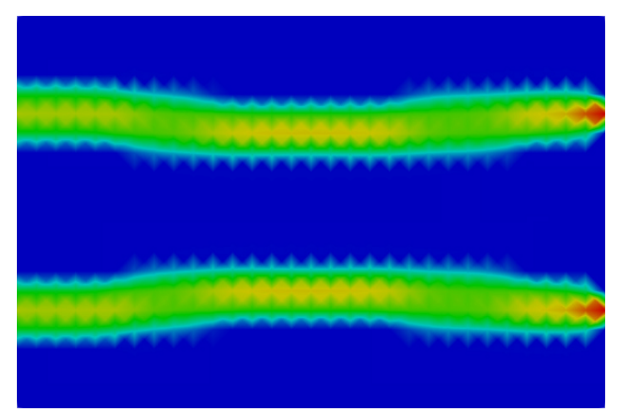

(b) $\operatorname{Re}=1.0$.

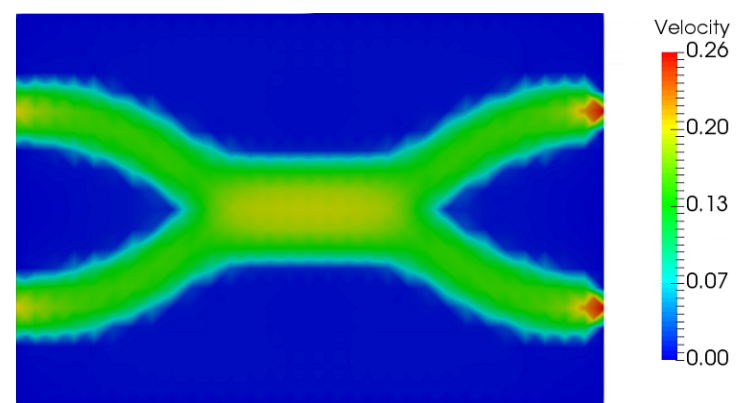

Figure 6.13 - Viscosity fields for double channel and Navier-Stokes non-Newtonian model (Carreau-Yasuda Viscosity Model).

(a) $\mathrm{Re}=0.1$.

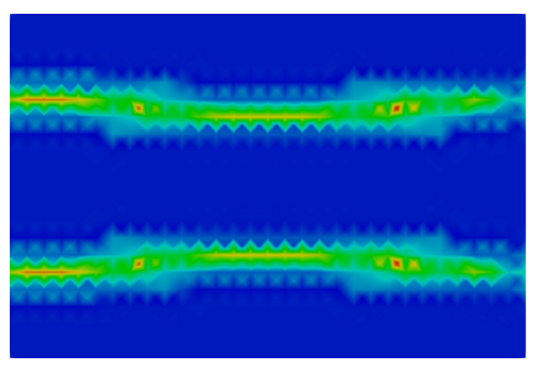

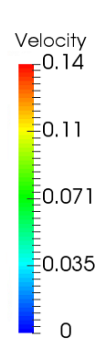

(b) $\operatorname{Re}=1.0$.
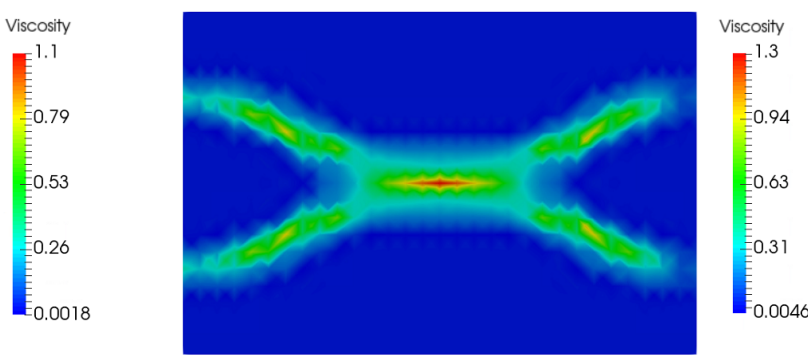

The results obtained are similar to the results presented in the work of Pingen and Maute (2010), where the total shear stress in the domain is also considered as the objective 
function.

It is possible to observe that the topologies may differ significantly when a constant or variable viscosity model is adopted. For higher velocity, thus higher shear rate, both cases presented a similar optimized design, with a merged channel. This is consistent with the Carreau-Yasuda model adopted for the shear-thinning effect, where in cases of higher shear rate, the behavior of blood is close to a Newtonian fluid with a value of viscosity that tends to the minimum $\mu_{\infty}=0.0035$ Pa.s.

In Fig. 6.13 it is possible to see that the viscosity field is consistent with the velocity field, given that at the center of the channels the viscosity presents a high value, indicating that at this point the shear rate is low. Also, the viscosity presents a lower value near the channel walls, indicating high shear rates, which is coherent with the no-slip condition and the boundary layer. The viscosity profile is in accordance with the interpolation used, described in Equation 3.3, given that in solid regions the viscosity presents a lower constant value.

\subsubsection{Obstacle}

The second implementation example is the pipe bend optimization problem with an obstacle, having energy dissipation as objective function. The corresponding design domain is shown in Figure 6.14. The volume constraint defines the total amount of fluid $V \leq 0.08 \pi L^{2}$. This value corresponds to a quarter of the volume of torus with inner radius $r_{i}=0.07 \mathrm{~m}$ and outer radius $r_{o}=0.09 \mathrm{~m}$ that fits exactly to the inlet and outlet, as suggested for the simple pipe bend problem in Borrvall and Petersson (2003). Parabolic velocity profiles are used at the inlet and outlet, where the velocity at the center presents a value $U_{\max }=0.01 \mathrm{~m} / \mathrm{s}$.

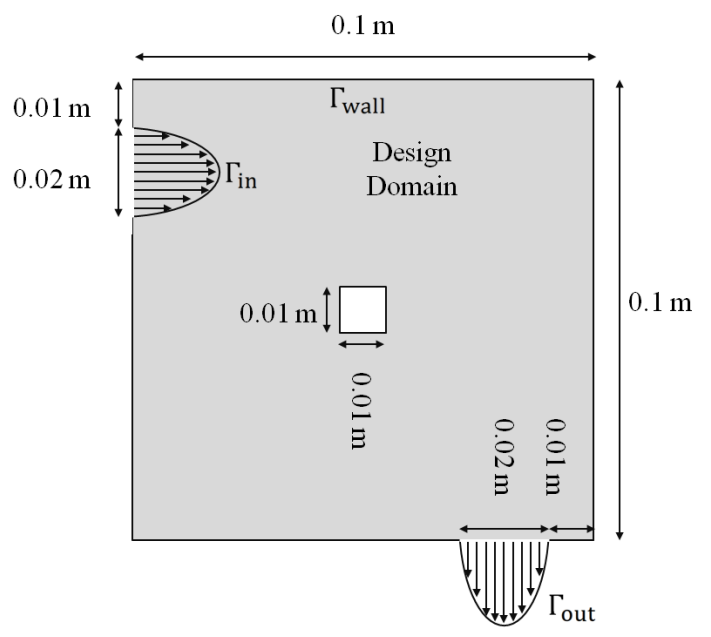

Figure 6.14 - Obstacle problem.

As a result, a comparison of topologies generated by considering Newtonian and 
Carreau-Yasuda viscosity model is presented in Figure 6.15, where the effect of modelling blood shear-thinning in the final design is visible.

Figure 6.15 - Optimized topologies for the obstacle problem.

(a) Newtonian viscosity.

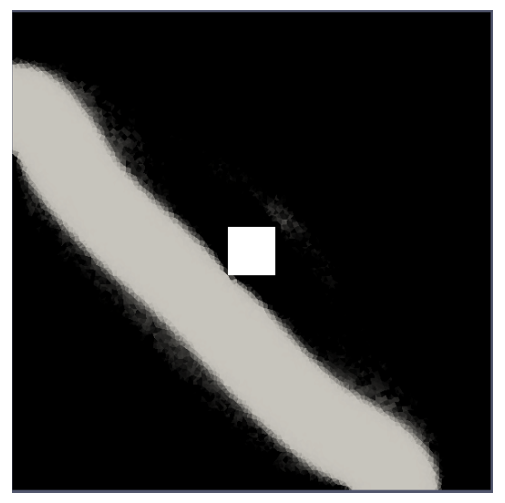

(b) Carreau-Yasuda.

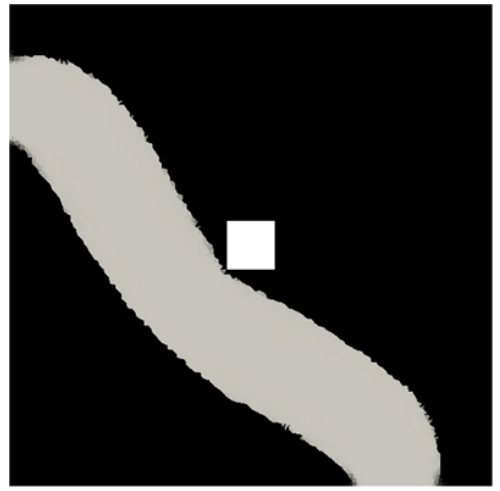

In Figure 6.16, the velocity profile and shear-thinning viscosity distribution for the non-Newtonian optimized topology are observed. The direct relation between them can be observed: in regions where the velocity gradients are lower, shear-stress has also a decreased value, an thus, the dynamic viscosity tends to the maximum adopted value $\mu_{0}=0.16$ Pa.s.

Figure 6.16 - Velocity and viscosity profiles for the Carreau-Yasuda model.

(a) Velocity.

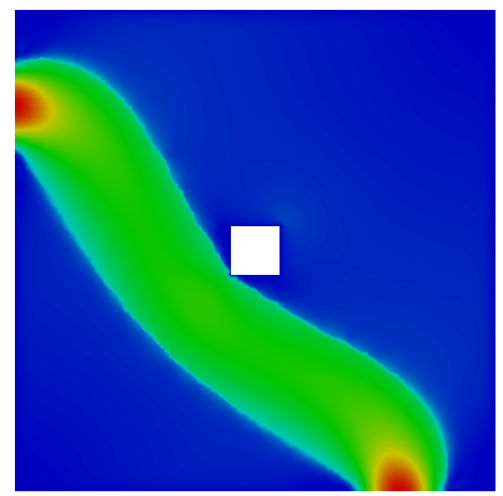

(b) Viscosity.

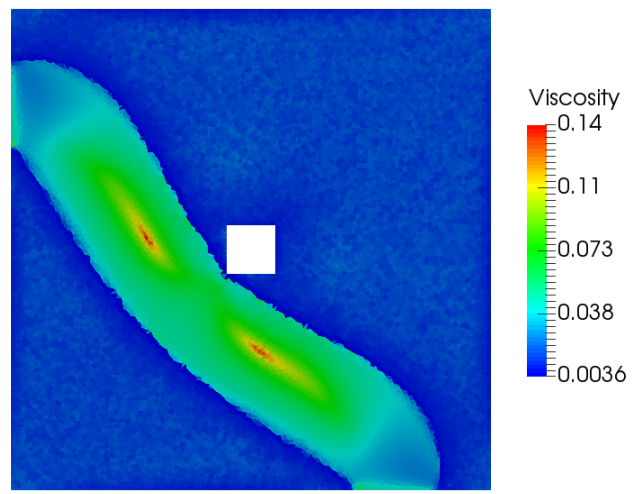

In addition, the value of Newtonian constant viscosity $\mu_{0}=0.0035 P$ a.s is displayed in the areas where the domain is filled with solid, due to the interpolation function employed (Eq. 3.2). The results obtained are similar to the results obtained by using the interpolation function from Equation 3.3 , however, when applying this pondering the convergence rate decreases. In the Newtonian model, the viscosity distribution is not shown because it is constant within the domain, both solid and fluid area present the same viscosity value. 


\subsubsection{Arterial Bypass}

Having verified the topology optimization process with the previous examples, the methodology is applied to practical problems, such as the arterial bypasses. The next sections illustrates the application of TOM to different cases of arterial bypasses.

\section{a) Fully blocked arterial bypass}

An important example of application of topology optimization to blood flow is the design of an arterial bypass, studied before by Abraham, Behr and Heinkenschloss (2005) and Zhang and Liu (2015), which consists in an artificial channel that allows the existence of flow in places where an artery is blocked. The objective function used aims to minimize the total shear stress of the fluid, since it is directly related with blood cell damage in artificial flows.

A parabolic inlet profile is adopted to simplify the examples, as seen in Section 6.1. This profile is adjusted by the formulation of Non-newtonian profile in the beginning of the channel. Thus, the optimization domain is defined in a region distant from the inlet.

The dimensions and boundary conditions for this problem are shown in Fig. 6.17. The highlighted area corresponds to the design domain, and the inferior central part corresponds to the blocked artery. Also, the original artery, represented by the lower channel with height $h$, is not modified during the process, so it is a fixed domain always filled with fluid. At the inlet, velocity profile is imposed with $U_{\max }=0.025 \mathrm{~m} / \mathrm{s}$ and at the outlet a zero stress condition is considered. The upper region is the domain where the bypass topology will be formed.

Figure 6.17 - Arterial bypass domain.

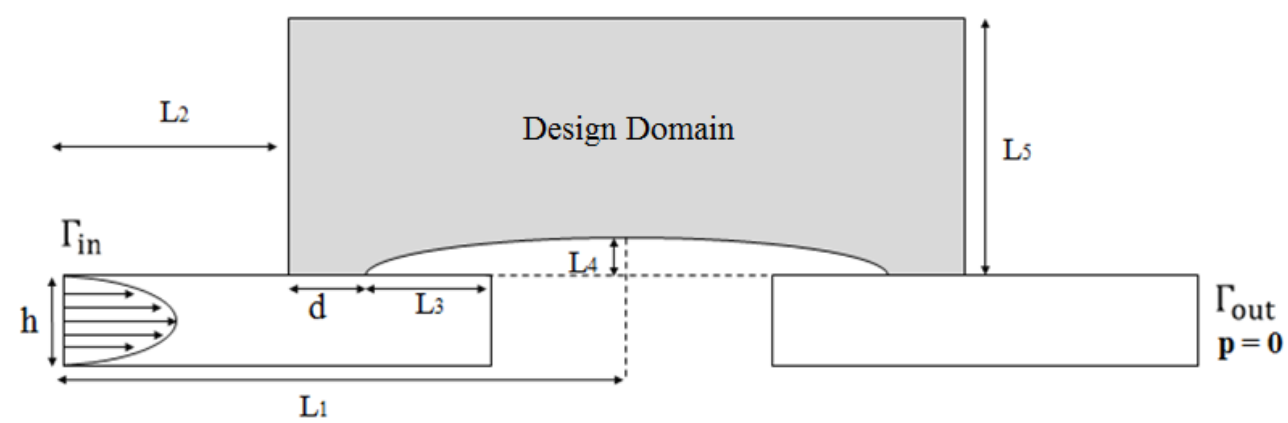

The adopted dimension are: $h=0.068 m, d=0.006 m, L_{1}=0.06 m, L_{2}=0.03 m$, $L_{3}=0.019 m, L_{4}=d / 4$, and $L_{5}=0.02 m$.

In this example the Carreau-Yasuda viscosity model is used without the porous interpolation on viscosity, due to the fact that without this interpolation the convergence is faster and the results are not influenced too much. A volume constraint of $33 \%$ of the total volume is used. Also, an initial guess similar to Abraham, Behr and Heinkenschloss 
(2005) is used, as shown in Fig 6.18.

Figure 6.18 - Initial guess for bypass topology optimization of fully blocked artery.

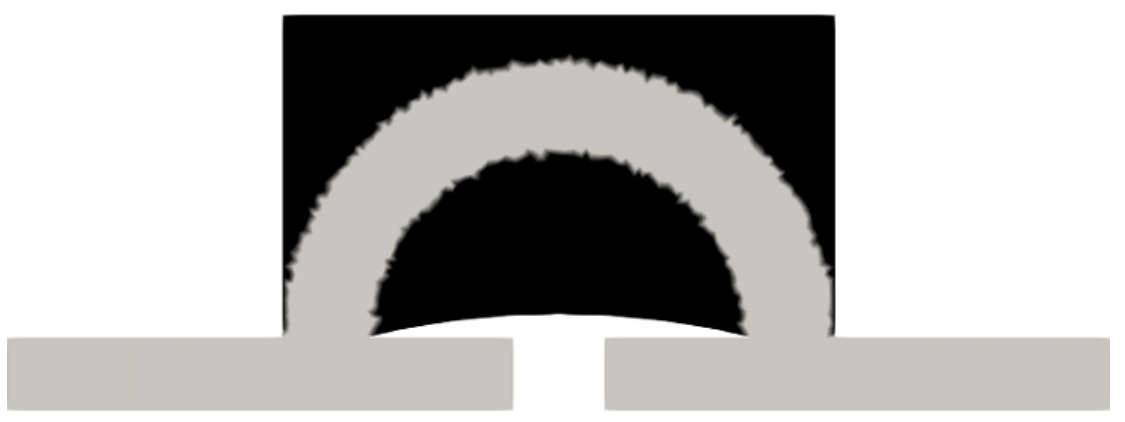

Figure 6.19 - Velocity and viscosity fields for bypass initial guess of fully blocked artery.

(a) Velocity Field.
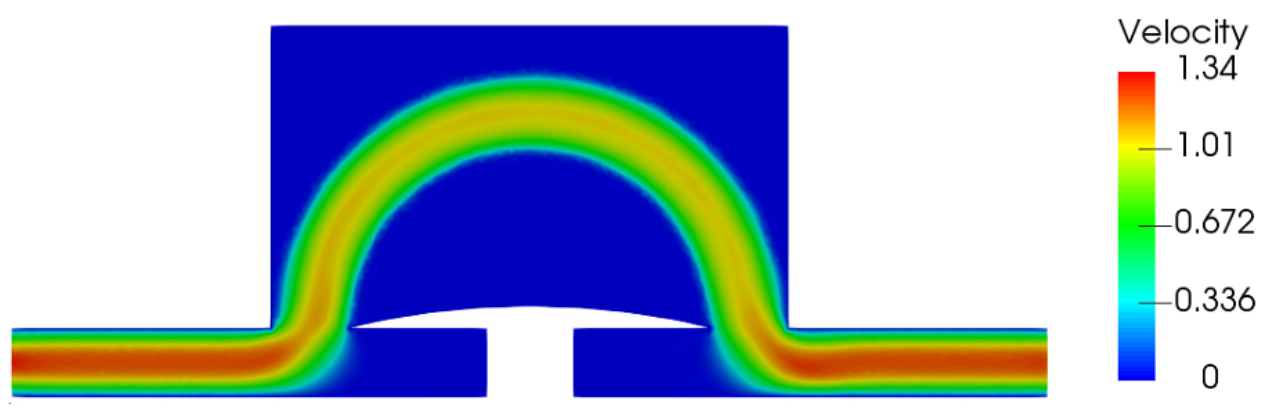

(b) Viscosity Field.

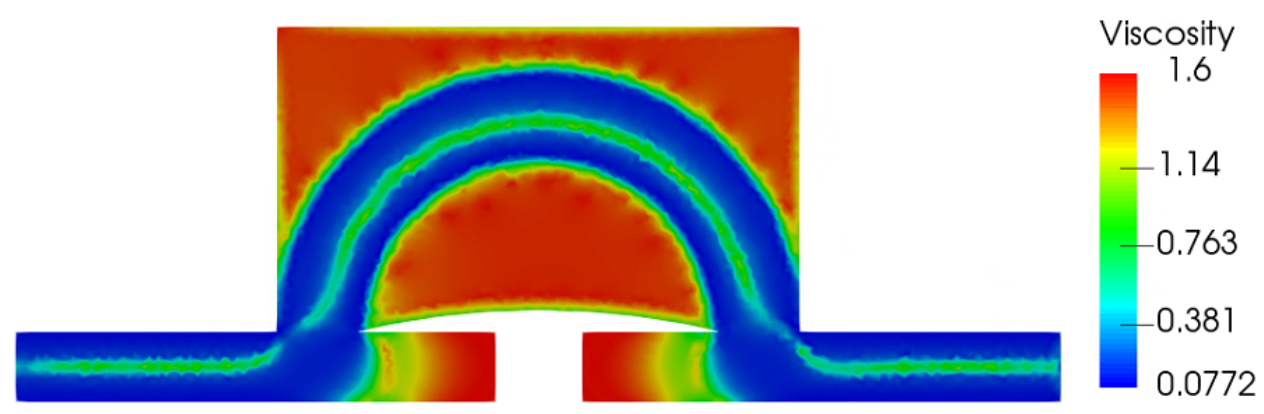

The final topology is shown in Fig.6.20. Here, it is possible to notice that the algorithm tends to create the shorter path between the inlet and outlet. This result was also observed by Zhang and Liu (2015). This indicates that this topology, juxtaposed to the wall, creates a lower energy dissipation value than the initial guess.

Figure 6.21 shows the velocity and viscosity fields for the optimized topology. In Fig. 6.21a, we observe that the overall velocity is lower in the bypass region when compared with the initial guess (Fig 6.19a). The viscosity field (Fig 6.21b) shows a higher viscosity within the channel, which signifies that the shear rate in the optimized channel is lower. Thus, from this conclusion we can infer that the initial topology (Fig.6.19b) has a higher shear rate inside the channel.

It is important to notice that, as cited before, the viscosity is not interpolated with 
respect to the design variable, thus, the solid regions on the viscosity fields present a low shear rate, hence, close to the upper value for viscosity $\left(\mu_{0}=0.16 P a \cdot s\right)$.

Figure 6.20 - Final topology for bypass optimization of fully blocked artery.

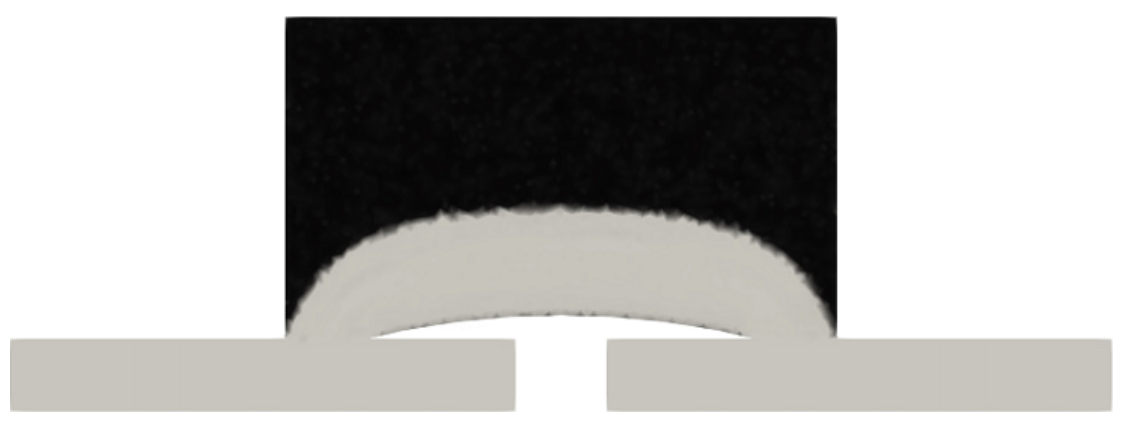

Figure 6.21 - Velocity and viscosity fields for bypass optimization of fully blocked artery.

(a) Velocity Field.

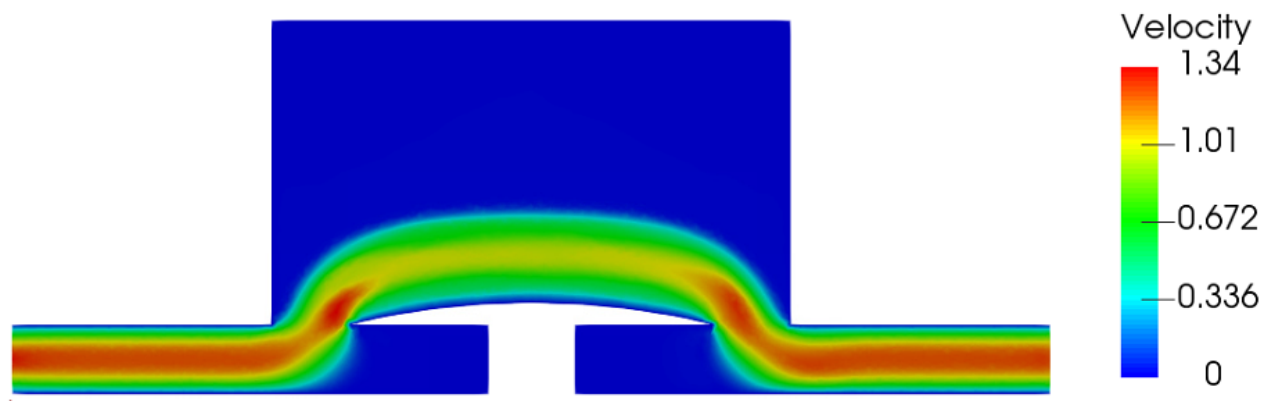

(b) Viscosity Field.

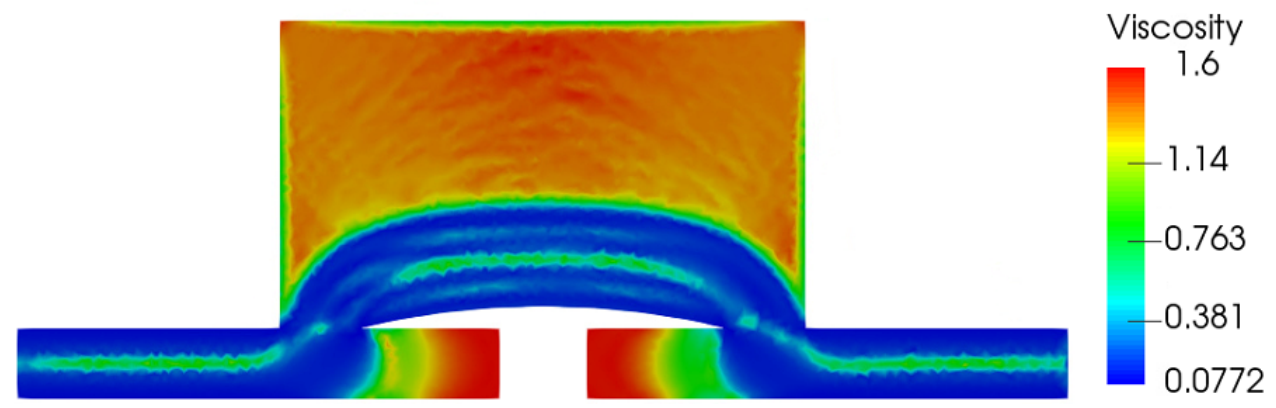

Figure 6.22 shows the convergence curve for the optimization and Fig.6.23 shows the topology evolution during the process. The functional value can be broken in two different parts, being the first identified as the shear stress $\left(\int_{\Omega} \frac{1}{2}\left(\nabla \mathbf{u}+\nabla \mathbf{u}^{T}\right):\left(\nabla \mathbf{u}+\nabla \mathbf{u}^{T}\right) d \Omega\right)$ and the second as the inertial velocity term $\left(\int_{\Omega} \alpha(\rho) \mathbf{u}^{2} d \Omega\right)$. As can be seen, initially the shear stress value increases as the volume decreases to attend the restriction. Also, the inertial part is initially very low, given that the domain do not present intermediary porosity regions, however, as the optimization proceeds the value grows and then decreases as the gray regions disappear.

The final functional values can be seen in Table 6.3. The final topology presents a improvement in shear stress of $42 \%$ while the inertial part increases, however the reduction 
of the shear stress is the main objective, thus the optimization is performed successfully.

Table 6.3 - Final Functional values for bypass optimization of fully blocked artery.

\begin{tabular}{lcccc}
\hline Case & Area $\left[\mathrm{cm}^{2}\right]$ & Objective Function & Shear Stress & Inertial Part \\
\hline Initial guess & 16.57 & 198.79 & 198.35 & 0.44 \\
Optimized & 15.63 & 129.65 & 115.47 & 14.18 \\
\hline
\end{tabular}

Figure 6.22 - Convergence curve for bypass optimization of fully blocked artery.

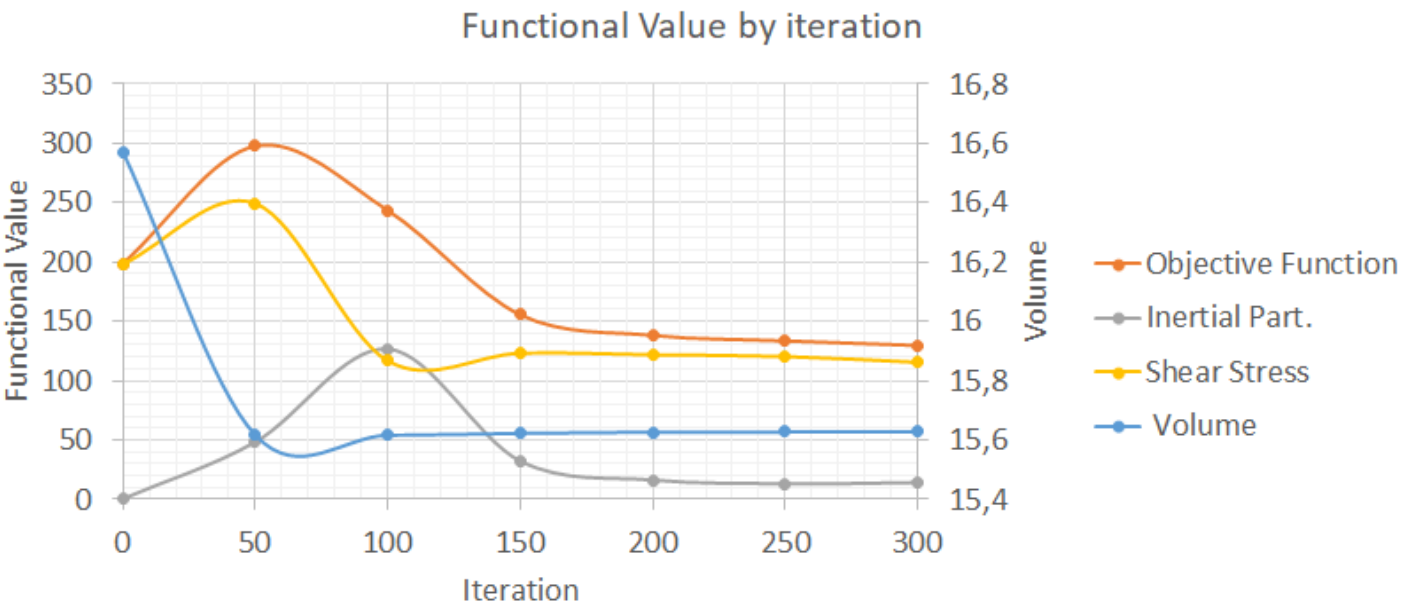

Figure 6.23 - Topology changes during bypass optimization of fully blocked artery.

(a) Iteration 0 .

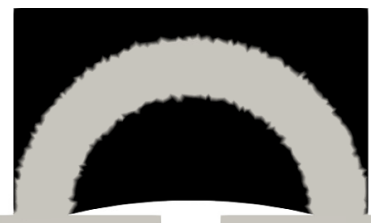

(c) Iteration 10 .

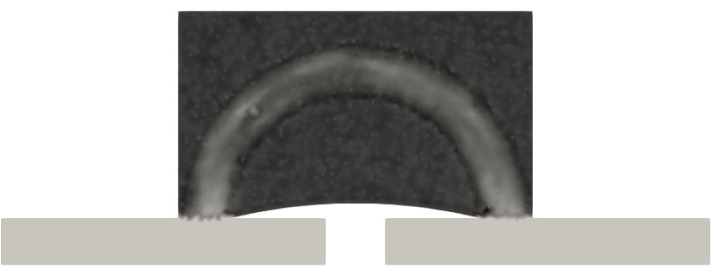

(e) Iteration 50.

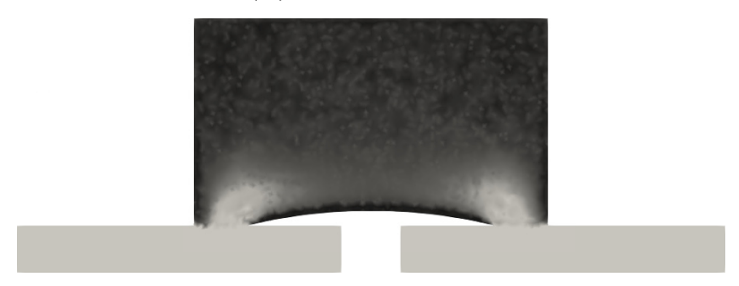

(b) Iteration 150 .

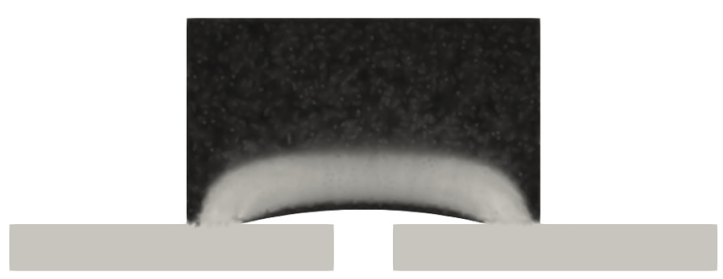

(d) Iteration 200 .

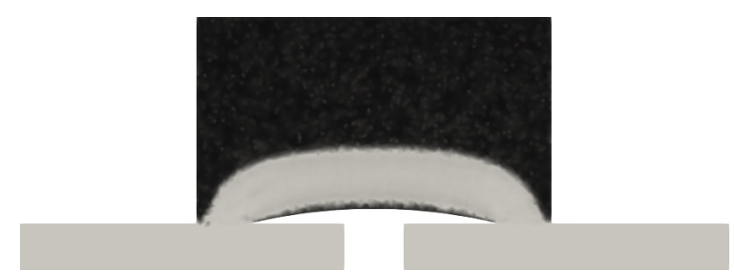

(f) Iteration 300.

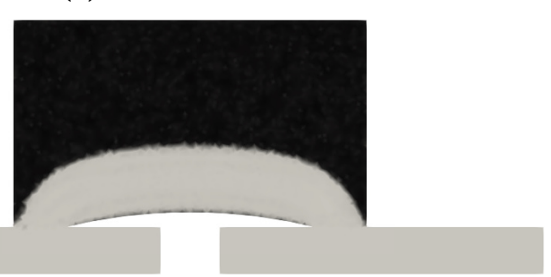




\section{b) Partial blocked arterial bypass}

The second case of an arterial bypass consists of a partial blocked artery (stenosed) in which another artery outlet is appended. Thus, in order to obtain a better design of the joint section between the host artery and the graft, both the host artery and graft outlets are set as the design objects. The objective function evaluated corresponds to minimizing the domain shear stress (Eq. 3.6). This application is also studied in the work of Zhang and Liu (2015) and the arterial bypass domain includes two inlets which are the host artery inlet and the graft inlet and one outlet referring to the host artery continuation.

Again, two parabolic inlet profiles are adopted and domain extensions are added to allow the flow development until the Non-Newtonian profile. The viscosity model adopted is the Carreau-Yasuda Non-Newtonian model without the interpolation with respect to the design variable. The dimensions and boundary conditions for this problem are shown in Fig. 6.24. The highlighted area corresponds to the design domain, which excludes the domain extension cited before.

This application is divided in three sub-cases, in which the inlet velocities are changed to represent different situations. The three cases are described in Table 6.4.

Table 6.4 - Cases division for bypass optimization of partial blocked artery.

\begin{tabular}{cccc}
\hline & Case 1 & Case 2 & Case 3 \\
\hline$u_{1}[\mathrm{~cm} / \mathrm{s}]$ & 10.5 & 15.75 & 5.25 \\
$u_{2}[\mathrm{~cm} / \mathrm{s}]$ & 10.5 & 5.25 & 15.75 \\
\hline
\end{tabular}

Figure 6.24 - Design domain and boundary conditions for stenosed artery bypass.

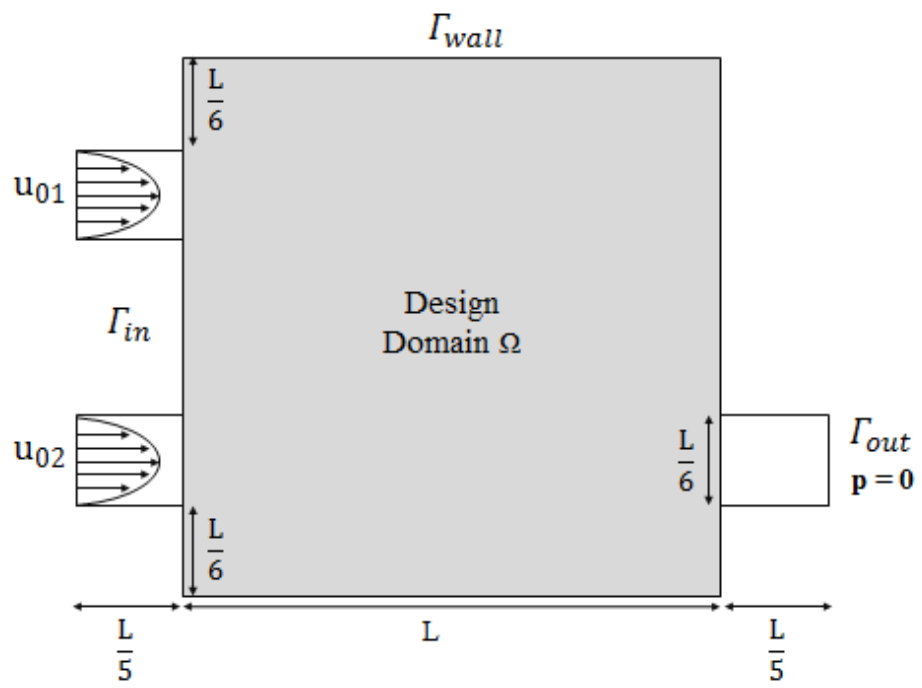


Case $1\left(u_{1}=10.5[\mathrm{~cm} / \mathrm{s}], u_{2}=10.5[\mathrm{~cm} / \mathrm{s}]\right)$

This case considers both artery and graft with the same flow rate, i.e., the same parabolic profiles with peak velocity of $10.5[\mathrm{~cm} / \mathrm{s}]$, which represents a Reynolds number of 100. The final topology is shown in Fig. 6.25. The velocity and viscosity fields are presented in Fig.6.27.

In this example we can see that both channels have similar widths close to the inlet. Observing the velocity field (Fig. 6.26) the channels unite midway in an angle close to the double channel results (Fig,6.11b).

The viscosity profile, shown in Fig. 6.27a, presents a higher value at the solid regions since velocity gradients are close to zero. At the fluid channel, the viscosity value is lower and apparently constant, however in Fig. 6.27b the viscosity profile is plotted with a reduced maximum value and it is possible to observe the non-Newtonian behavior of the fluid. At the walls, where velocity gradient and also shear rate is higher, viscosity has a lower value. At the center of the fluid channel, the opposite effect is observed, velocity gradients are lower and, thus, the viscosity value increases sightly.

Figure 6.25 - Final topology for stenosed artery optimization (Case 1).

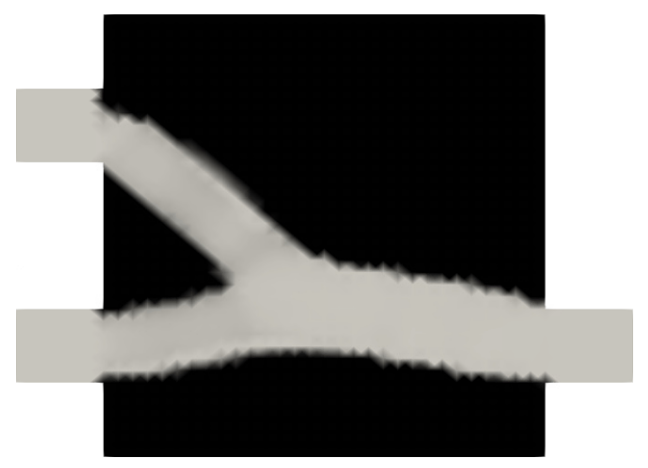

Figure 6.26 - Velocity field for stenosed artery optimization (Case 1).

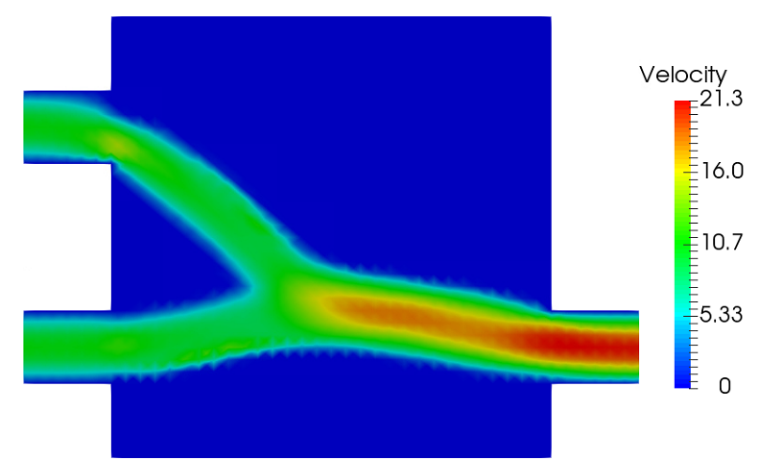


Figure 6.27 - Viscosity field for stenosed artery optimization (Case 1).

(a) Viscosity Field.

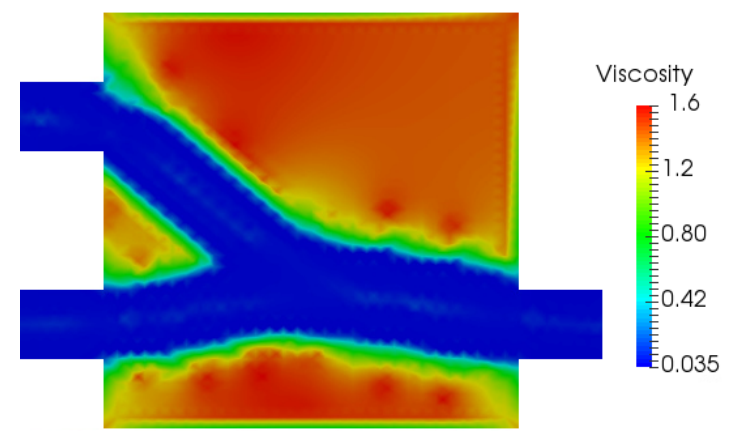

(b) Viscosity field inside the channel.

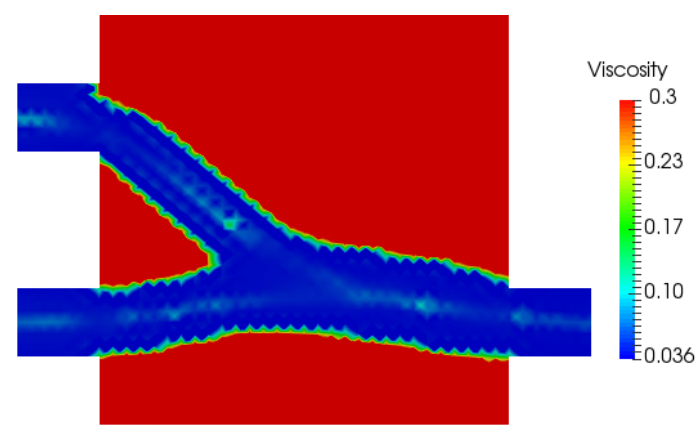

Case $2\left(u_{1}=5.25[\mathrm{~cm} / \mathrm{s}], u_{2}=15.75[\mathrm{~cm} / \mathrm{s}]\right)$

This case considers that the upper artery is obstructed and graft must provide the remaining flow to maintain the flow rate at the outlet, i.e., the parabolic profiles have different peak values of $5.25[\mathrm{~cm} / \mathrm{s}]$, in the upper inlet, and $15.75[\mathrm{~cm} / \mathrm{s}]$ in the lower inlet. The final topology is shown in Fig. 6.28. The velocity and viscosity fields are presented in Fig 6.30.

In this example we can see that the high velocity channel dominates the optimization, in a way that the final topology is almost a straight channel with a small collaboration of the upper inlet in the contour, promoting a small curvature in the lower part of the channel near where it unites.

The final topology seems discontinuous near the upper inlet, however, the results is correct, given that the small extensions are considered fixed as fluid and do not enter the optimization. If this extension is part of the optimization domain, a curve consistent with the velocity profile would be created. The velocity field (Fig.6.29) is coherent with the topology and the fluid only flows where the topology enables. The viscosity profile (Fig. 6.30 is consistent with the characteristics of fluid flow at this optimized topology and again, the profile is shown in two different value ranges so that the viscosity inside the fluid can be observed.

Figure 6.28 - Final topology for stenosed artery optimization (Case 2).

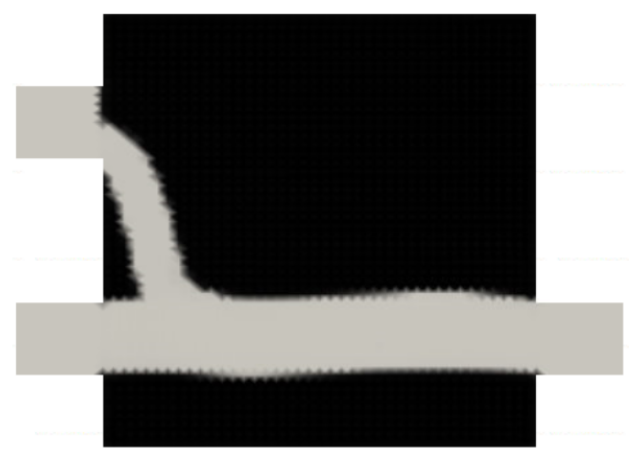


Figure 6.29 - Velocity field for stenosed artery optimization (Case 2).

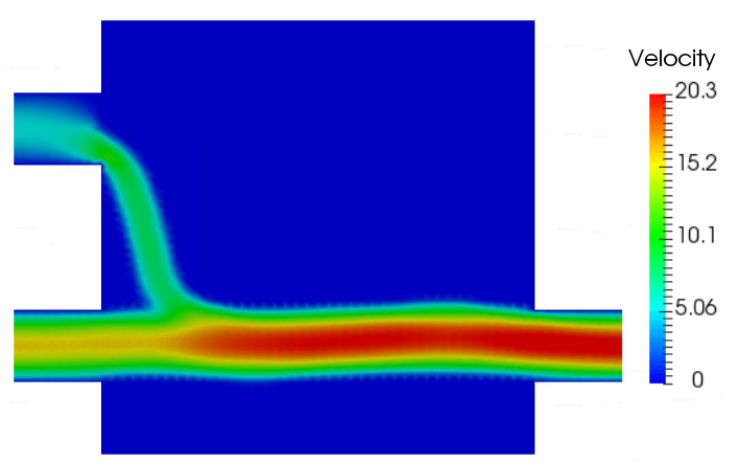

Figure 6.30 - Viscosity field for stenosed artery optimization (Case 2).

(a) Viscosity Field.

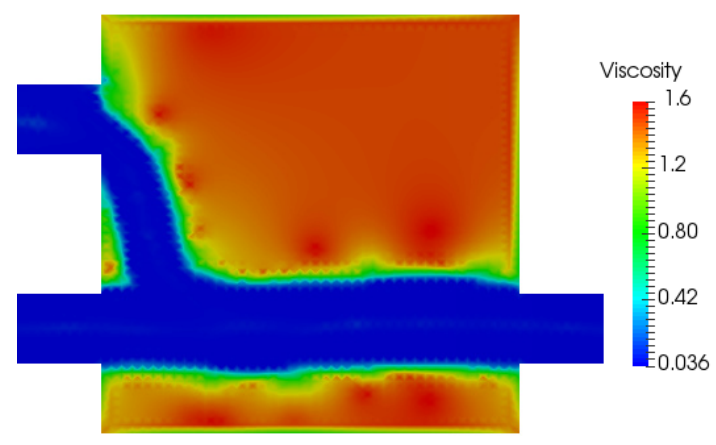

(b) Viscosity field inside the channel.

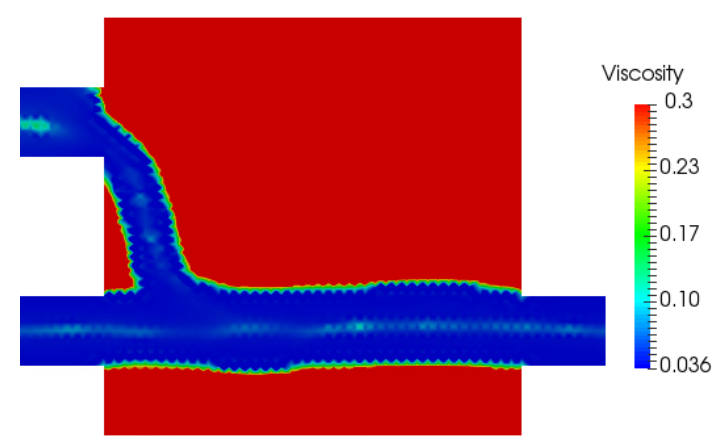

Case $3\left(u_{1}=15.75[\mathrm{~cm} / \mathrm{s}], u_{2}=5.25[\mathrm{~cm} / \mathrm{s}]\right)$

This case is the opposite of the Case 2, it considers that the lower artery is obstructed and graft must provide the remaining flow to maintain the flow rate at the outlet, i.e., the parabolic profiles have different peak values of $15.75[\mathrm{~cm} / \mathrm{s}]$, in the upper inlet, and $5.25[\mathrm{~cm} / \mathrm{s}]$ in the lower inlet. The final topology is shown in Fig. 6.31. The velocity and viscosity fields are presented in Fig.6.33.

The same effect of apparently discontinuity occurs in the final topology near the inlet (Fig.6.31), however, as explained before, the domain extensions are always fluid and inside the design domain the fluid only flows where the topology permits.

Even though the example is very similar to Case 2, the velocity profile (Fig.6.32) shows that the union of the channels occur farther from the inlet and promotes a union angle similar to the double channel result (Fig 6.9b). As the cases presented before, the viscosity (Fig. 6.33 has a higher value in the solid regions. In Fig. 6.33b it is shown that the central region of the fluid channel presents a sightly higher value then the rest of the channel, which indicates lower shear rate and a minimum value at the walls, where the velocity gradient is higher. 
Figure 6.31 - Final topology for stenosed artery optimization (Case 3).

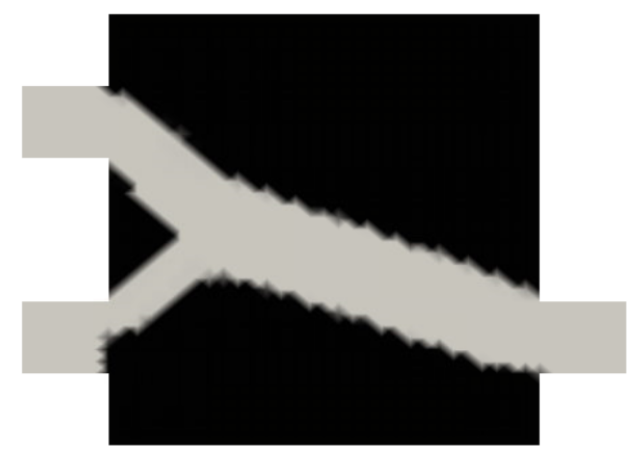

Figure 6.32 - Velocity field for stenosed artery optimization (Case 3).

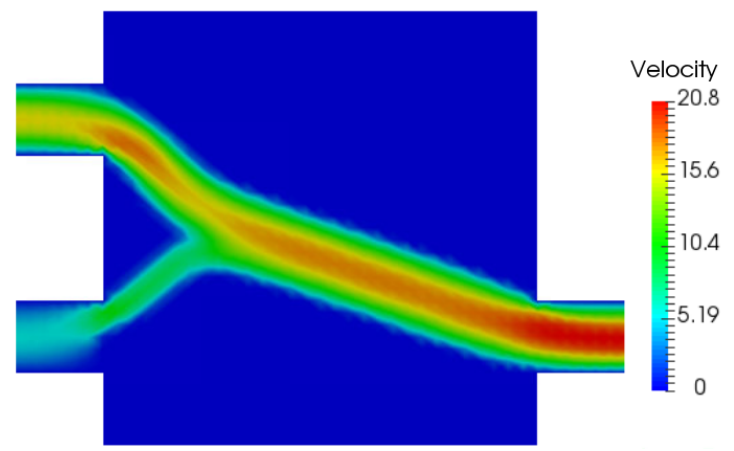

Figure 6.33 - Viscosity field for stenosed artery optimization (Case 3).

(a) Viscosity Field.

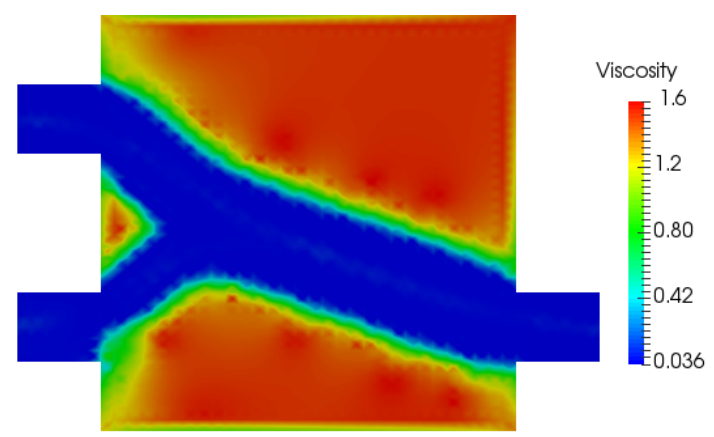

(b) Viscosity field inside the channel.

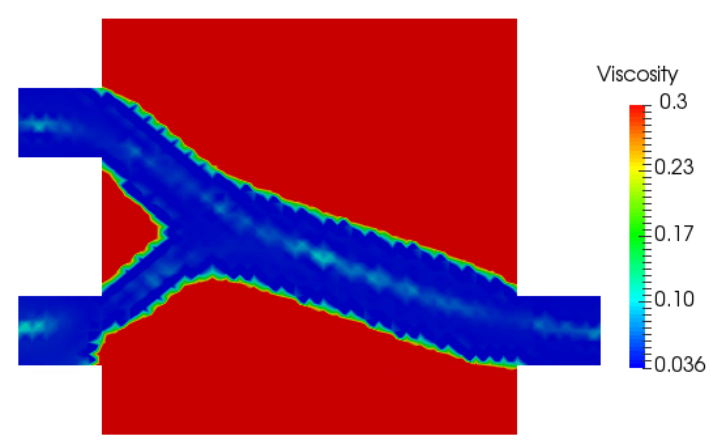

\subsubsection{Bypass considering Vorticity}

The vorticity is an objective function of interest when designing devices that operates with blood, because it has a direct relation with the blood stagnation, which causes a agglomeration of blood cells in regions with high re-circulation. The cell agglomeration can cause thrombosis, i.e., the agglutination can detach from the re-circulation zone and go through the circulatory system blocking a capillary artery causing more serious diseases.

This example considers the same design domain from Fig.6.17, Also, the initial guess is the same of Fig.6.18. The pure vorticity functional presents a particularity, being that the optimized topology is formed by a "gray" domain. The intermediary porosity causes 
the reduction in the velocity and, with this, a reduction in the vorticity value, as can be seen in Tab.6.5. This "gray" topology is shown in Fig. 6.34.

Table 6.5 - Functional values for vorticity optimization.

\begin{tabular}{lccc}
\hline Topology & Volume & Vorticity & Inertial Part. \\
\hline Initial Guess (Fig. .6 .18$)$ & 16.57 & 41.41 & - \\
Gray Topology (Fig. .6 .34$)$ & 15.57 & 6.40 & - \\
Final Topology (Fig. .6 .35$)$ & 15.60 & 20.96 & 2.32 \\
\hline
\end{tabular}

Figure 6.34 - Gray final topology for pure vorticity optimization.

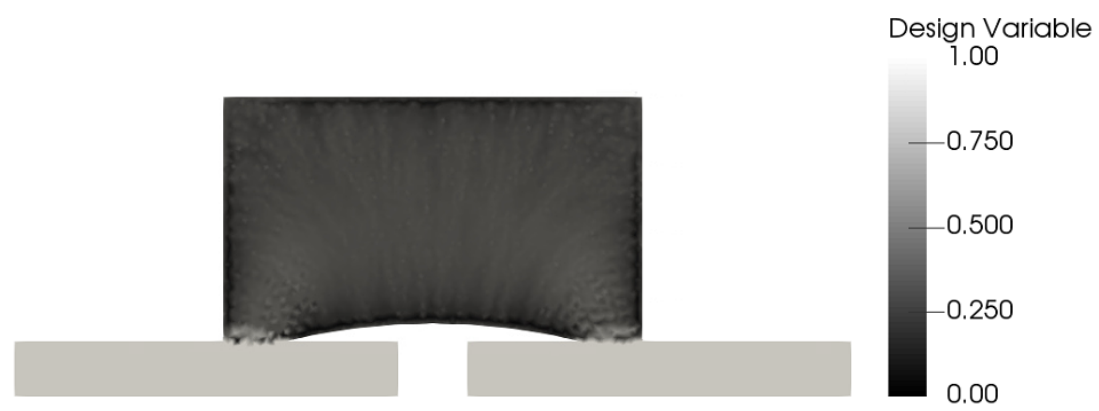

This gray domain represents a material with intermediary porosity and it is not possible to build. Thus in order to circumvent this problem, a term is added to the functional representing the flow within the porous media, as shown in Eq. 6.1. This added term implies in the minimization of the velocity in low porosity regions, thus promoting a continuous topology.

$$
J_{v}(\mathbf{u})=\int_{\Omega}|\nabla \times \mathbf{u}|^{2} d \Omega+\omega_{v} \int_{\Omega} \alpha(\rho) \mathbf{u}^{2} d \Omega
$$

where $\omega_{v}$ is a weight coefficient used to calibrate the optimization direction (adopted value is 0.3 ). The added term is the same present in the energy dissipation functional and refers to the inertial part.

The final topology obtained with this new objective function is shown in Fig 6.35. The topology is very similar to the one obtained with the energy dissipation functional (Fig.6.20), which may indicate that the inertial term dominated the optimization. However, observing the convergence curve from Fig. 6.36 it is possible to notice that the vorticity part is also minimized. From Tab. 6.5, the vorticity is reduced to approximately $49.38 \%$ of the initial guess in the optimized topology. 
Figure 6.35 - Final topology for vorticity optimization.

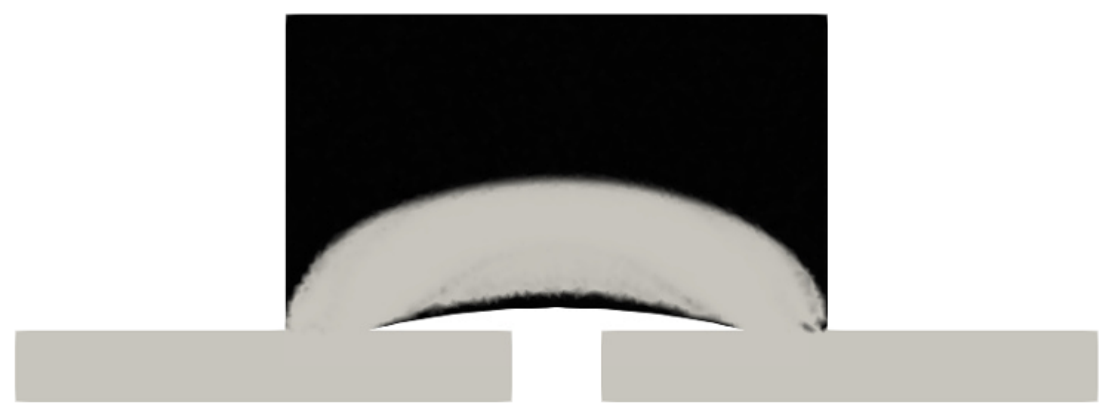

Figure 6.36 - Convergence curve for bypass optimization considering vorticity functional with added inertial part.

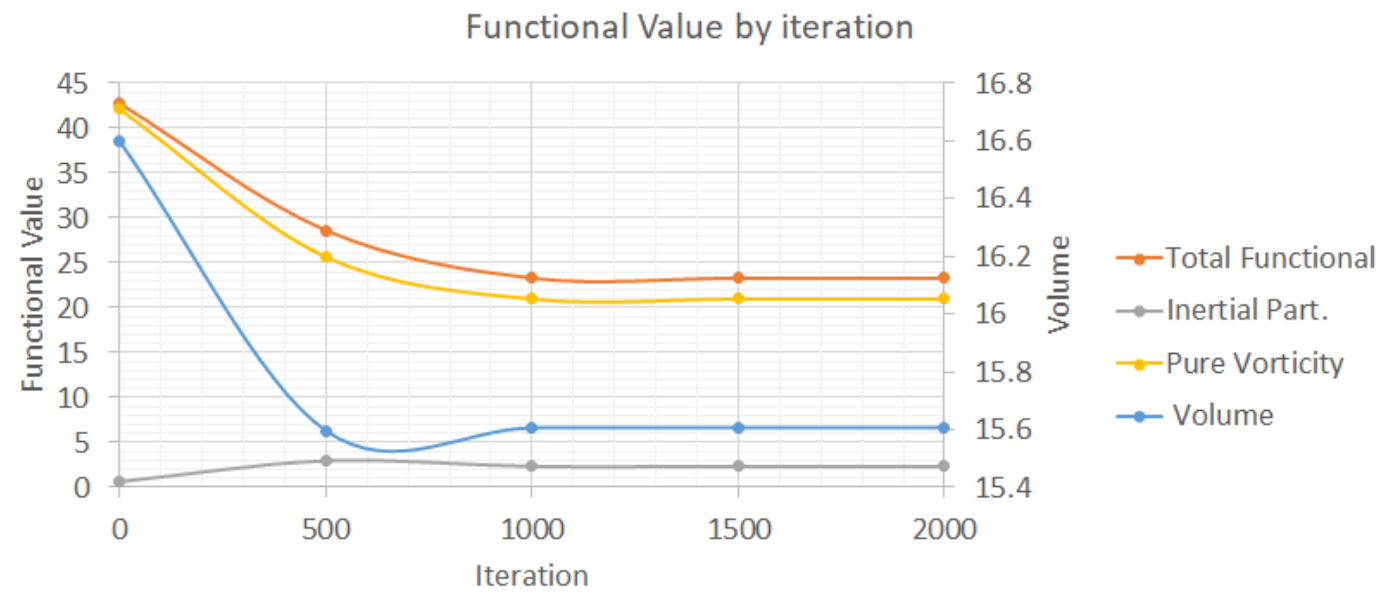

\subsubsection{Multi-objective bypass}

The previous sections introduced the optimization considering different objective functions, such as, energy dissipation, shear rate and vorticity. However, it is possible to optimize the bypass by considering a weighted union between these functionals, as described in Section 3.4.4. Hence, this section presents the optimization considering both the vorticity and the energy dissipation functionals.

This example considers the same design domain from Fig.6.17. Also, the initial guess is the same of Fig. 6.18. The weights of $w_{e}=0.5$ and $w_{v}=0.5$ are used, i.e., the final functional has the same weight for both energy dissipation and vorticity, respectively. However, the vorticity has an additional multiplier $\beta_{k}$, used to normalize the difference in magnitude of the two functions, as described in Section 4.5 .

The final topology is shown in Fig. 6.37. Comparing this topology with the one obtained in the vorticity optimization (Fig. 6.35) we can see that the channel is more detached from the boundary. 
Figure 6.37 - Final topology considering the multi-objective functional.

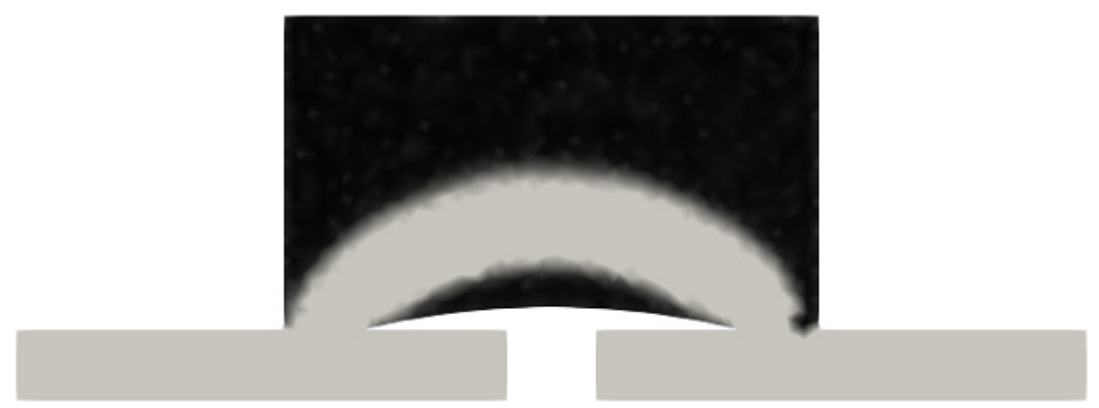

The convergence curve and the evolution of the topology during the optimization can be seen in Fig.6.38 and Fig 6.39, respectively. Also, the functional values for the final topology is shown in Table 6.6 .

Comparing the vorticity value from Tab 6.6 to the one from the vorticity optimization (Tab.6.5) we can see that the value is $25 \%$ higher in the multi-objective case. This indicates that the introduction of the viscous dissipation term in the objective function introduces a pareto behavior, i.e., the minimization of the viscous dissipation occurs in detriment of the vorticity. However, the final vorticity is still $33 \%$ lower than the initial guess, which indicates that the optimization succeeded and, therefore, this multi-objective optimization scheme is a viable option to minimize multiple functions.

Table 6.6 - Functional values for optimization considering the multi-objective functional.

\begin{tabular}{lccccc}
\hline Topology & Volume & Total & Viscous Dissip. & Inertial Part. & Vorticity \\
\hline Initial Guess (Fig.6.18) & 16.62 & 22.33 & 5.57 & 1.61 & 37.49 \\
Vorticity Opt. (Fig.6.35) & 15.60 & - & - & 2.32 & 20.96 \\
Multi-obj. Opt. (Fig.6.37) & 15.69 & 14.68 & 2.93 & 1.40 & 25.03 \\
\hline
\end{tabular}

Figure 6.38 - Convergence curve for optimization considering the multi-objective functional.

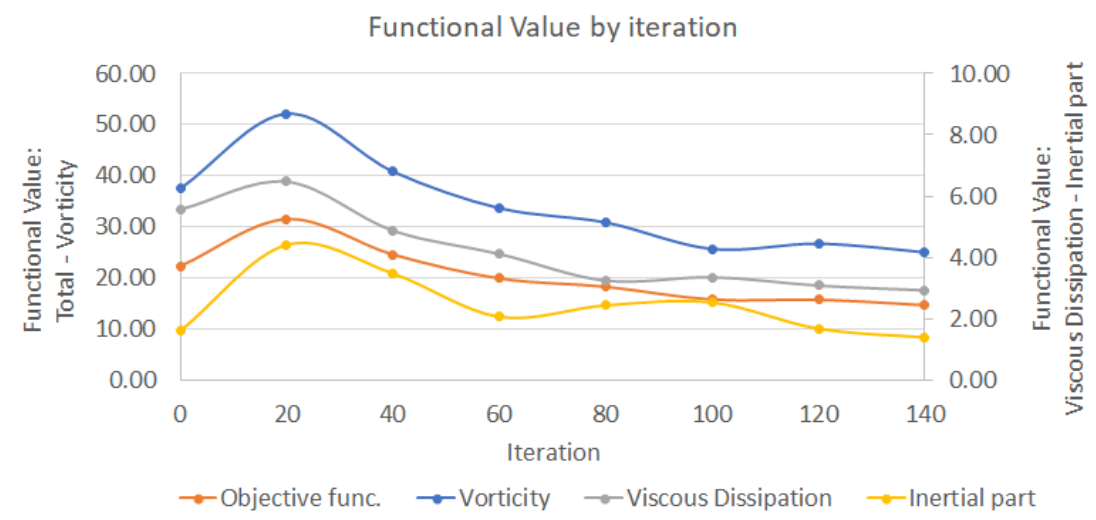


Figure 6.39 - Topology changes during bypass optimization considering the multi-objective functional.

(a) Iteration 20 .

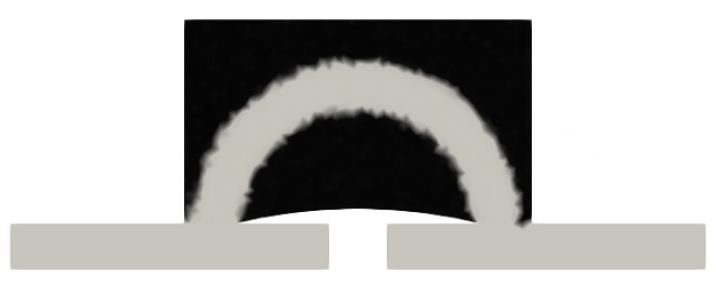

(c) Iteration 60.

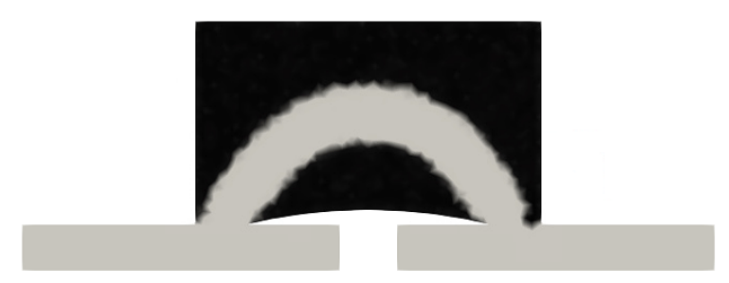

(b) Iteration 120 .

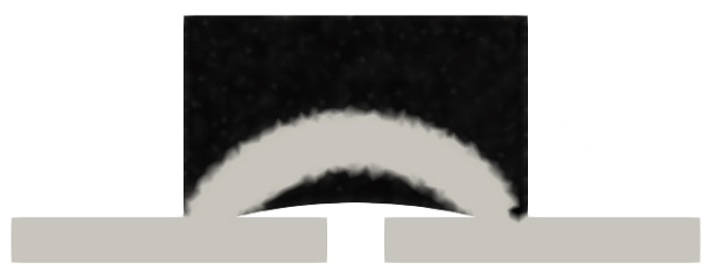

(d) Iteration 140.

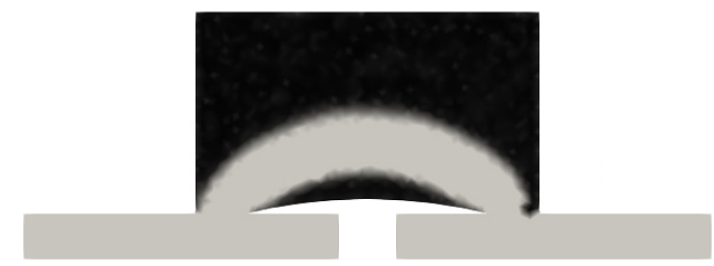

\subsubsection{D Difuser}

This example is presented to illustrate how the same methodology can be extended to 3D domains. It covers a 3D convergent diffuser designed by using the Navier-Stokes equations along with the Non-Newtonian Carreu-Yasuda model. The 3D example is rather simple, however it takes a much longer time to converge than the $2 \mathrm{D}$ problems. For example, this case took 27 hours to complete.

The objective function evaluated for this case is to minimize energy dissipation, which corresponds to pressure drop at the diffuser. A parabolic velocity profile is adopted at the inlet and zero stress condition at the outlet. Domain extensions are added to permit the flow development until the Non-Newtonian profile. The dimensions and boundary conditions for this problem are shown in Fig. 6.40. The highlighted area corresponds to the design domain, which excludes the domain extension cited before. 
Figure 6.40 - Design domain and boundary conditions for the 3D convergent diffuser.

(a) Domain Dimensions.

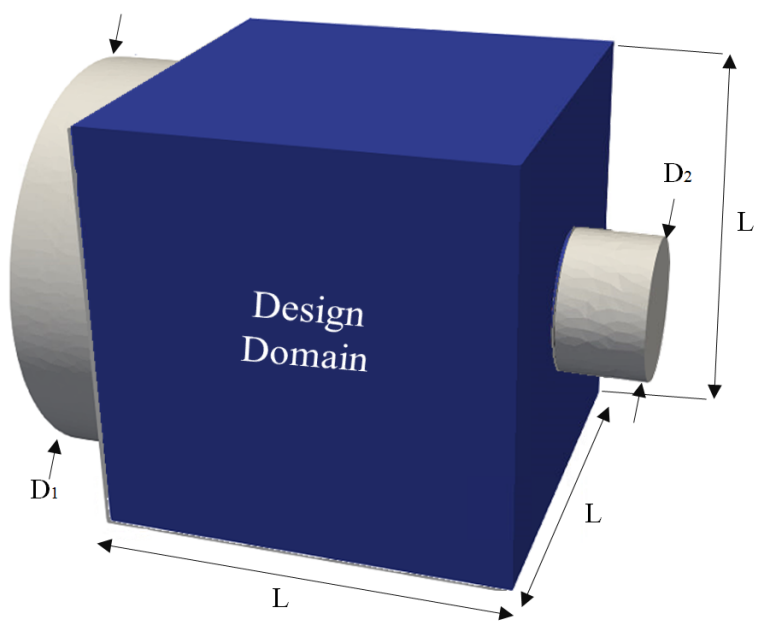

(b) Boundary Conditions.

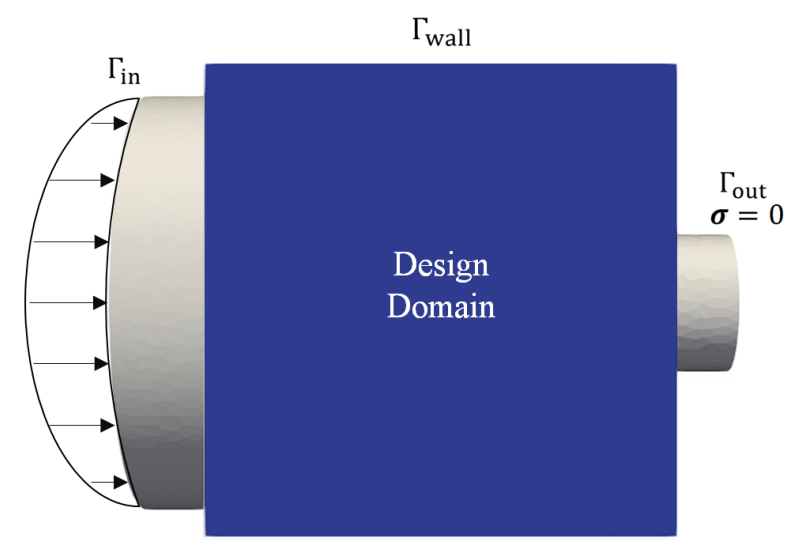

The final topology can be seen in Fig.6.41, where blue represents solid $(\rho=0)$ and red represents fluid $(\rho=1)$. The velocity and viscosity fields are shown in Fig. 6.42 and Fig.6.43, respectively. It is possible to notice that the methodology can be extended to the $3 \mathrm{D}$ cases. Also, observing the velocity field (Fig $6.42 \mathrm{~b})$ we can see that the velocity is minimized in the solid regions.

Figure 6.41 - Final topology for the 3D convergent diffuser optimization.

(a) Isometric view.

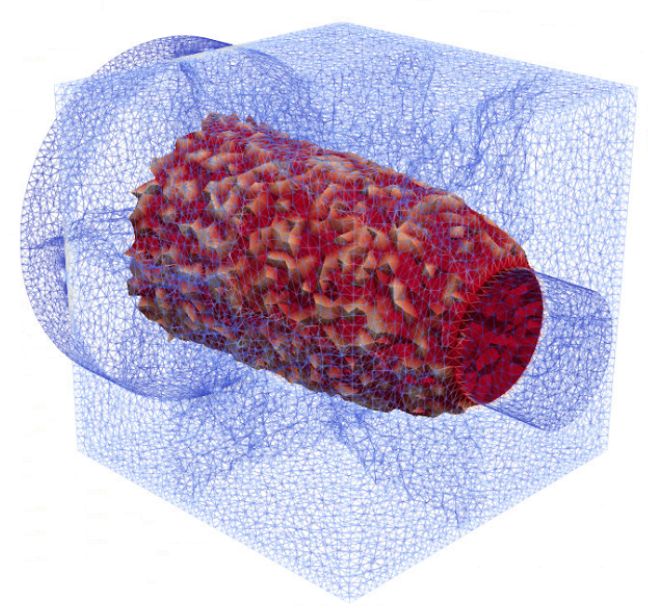

(b) Slice view.

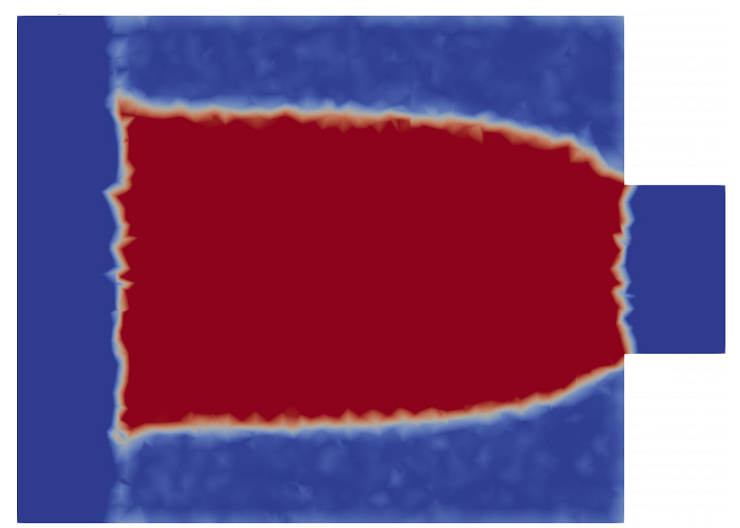


Figure 6.42 - Velocity field for optimized 3D convergent diffuser.

(a) Isometric view.

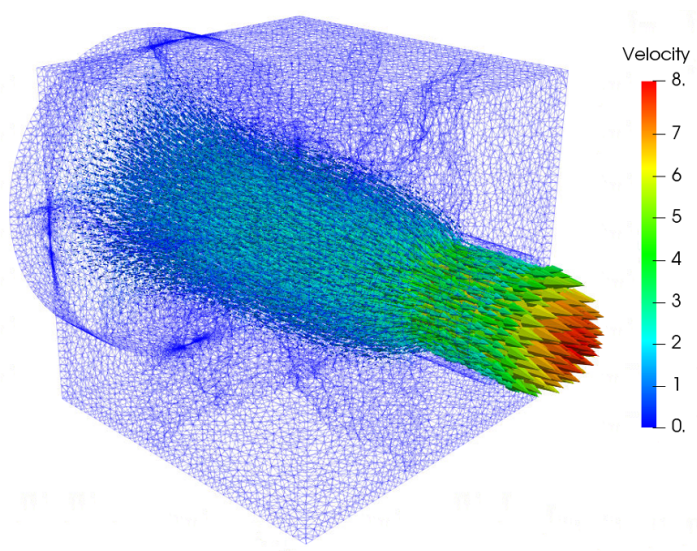

(b) Slice view.

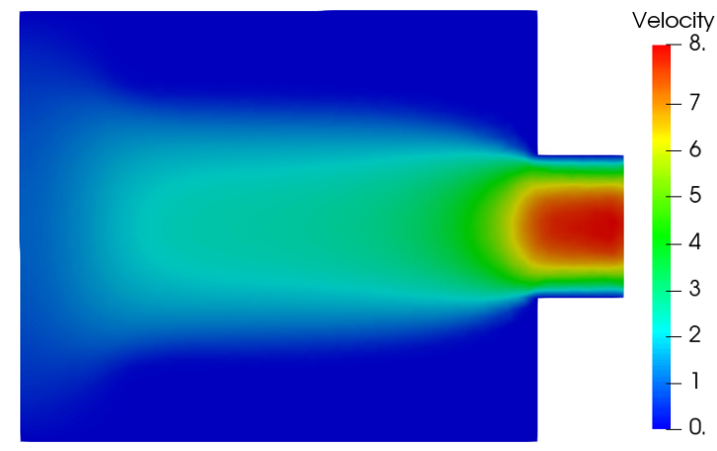

Figure 6.43 - Viscosity field for optimized 3D convergent diffuser.

(a) Isometric view.

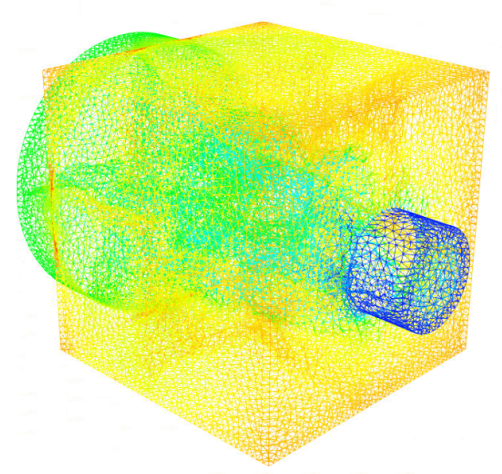

(b) Slice view.

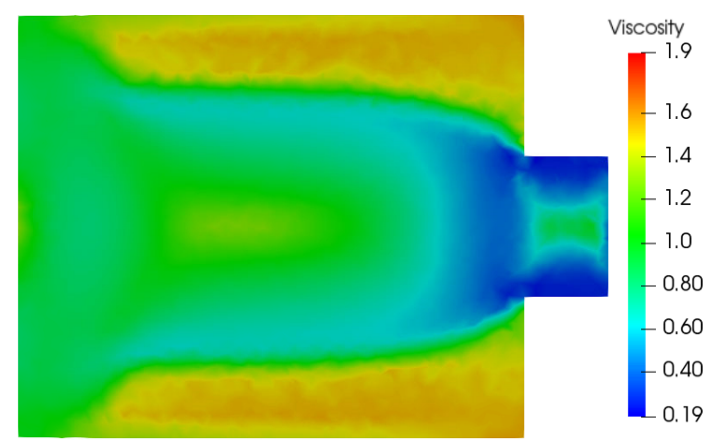




\section{CONCLUSIONS}

A methodology for design channel for non-Newtonian fluid flow based on topology optimization is presented. Carreau-Yasuda shear thinning model is coupled with the Navier-Stokes equations to represent more accurately non-Newtonian effects in blood flow.

In topology optimization for Newtonian fluids, a porous material model is used to allow a continuous transition between fluid and solid, where the inverse permeability $\alpha$ is interpolated as a function of the design variable $\rho$. For non-Newtonian fluids, some authors suggest to adapt this material model by adding an interpolation function of the dynamic viscosity $\mu$, which is also dependent of the pseudo-density $\rho$. In the examples studied, the convergence of the optimization algorithm was faster when only inverse permeability interpolation was employed.

It is also verified that optimization considering only shear stress or vorticity functional results in a "gray" domain, where many regions present an intermediate porosity value. Thus, it is necessary to add a term correspondent to minimization of flow thought the porous media in the objective function in order to achieve a discrete domain distribution.

The results show that the numerical formulation adopted is consistent with the literature, obtaining non-intuitive solutions that minimize the fluidic system dissipation of energy, shear stress or vorticity and satisfy the volume constraint imposed.

The optimized topologies differ significantly according to the fluid model used, Newtonian or shear-thinning, specially for higher velocities. This justifies the need to model blood as a non-Newtonian fluid and analyze a more complex system.

It is also shown that the FEniCS software library, the Dolfin-adjoint package and the IPOpt optimizer make a valid tool to implement the finite element method and optimization algorithm in a more automated way, simplifying the coding and application of topology optimization to fluidic problems.

\subsection{Future Work}

As future work, it is suggested to extend these studies to the optimization of rotary systems, which can be employed in another important bio-medical device for conducting blood, that is the ventricular assist device pump.

Also, for applications outside Bio-engineering field, it might be useful to explore the influence of non-Newtonian shear-thickening behavior, where, opposite to what occurs with blood, the dynamic viscosity value increases with higher velocity gradients. 


\section{REFERENCES}

AAGE, N.; POUlSEN, T.; GERSBORG-HANSEN, A.; SIGMUND, O. Topology optimization of large scale stokes flow problems. Structural and Multidisciplinary Optimization, Springer-Verlag, v. 35, n. 2, p. 175-180, 2008. ISSN 1615-147X. Available from Internet: <http://dx.doi.org/10.1007/s00158-007-0128-0>. Cited in page 26.

ABRAHAM, F.; BEHR, M.; HEINKENSCHLOSS, M. The effect of stabilization in finite element methods for the optimal boundary control of the oseen equations. Finite Elements in Analysis and Design, v. 41, n. 3, p. 229 - 251, 2004. ISSN 0168-874X. Available from Internet: <http://www.sciencedirect.com/science/article/pii/ S0168874X04000861>. Cited in page 31.

ABRAHAM, F.; BEHR, M.; HEINKENSCHLOSS, M. Shape optimization in steady blood flow: A numerical study of non-newtonian effects. Computer Methods in Biomechanics and Biomedical Engineering, v. 8, n. 2, p. 127-137, 2005. PMID: 16154876. Available from Internet: <http://dx.doi.org/10.1080/10255840500180799> Cited 9 times in pages 14, 15, 22, 23, 27, 31, 48, 57, and 58.

ALNæS, M.; BLECHTA, J.; HAKE, J.; JOHANSSON, A.; KEHLET, B.; LOGG, A.; RICHARDSON, C.; RING, J.; ROGNES, M.; WELLS, G. The fenics project version 1.5. Archive of Numerical Software, v. 3, n. 100, 2015. ISSN 2197-8263. Available from Internet: <http://journals.ub.uni-heidelberg.de/index.php/ans/article/view/20553>. Cited in page 44 .

BEnDSOE, M.; SIGMUND, O. Topology Optimization: Theory, Methods and Applications. Springer, 2003. ISBN 9783540429920. Available from Internet: $<$ http://books.google.com.br/books?id=NGmtmMhVe2sC $>$. Cited 2 times in pages 15 and 25.

BENDSOE, M. P.; KIKUCHI, N. Generating optimal topologies in structural design using a homogenization method. Comput. Methods Appl. Mech. Eng., Elsevier Sequoia S. A., Lausanne, Switzerland, Switzerland, v. 71, n. 2, p. 197-224, nov. 1988. ISSN 0045-7825. Available from Internet: <http://dx.doi.org/10.1016/0045-7825(88)90086-2>. Cited in page 25 .

BOEK, E. S.; CHIN, J.; COVENEY, P. V. LATTICE BOLTZMANN SIMULATION OF THE FLOW OF NON-NEWTONIAN FLUIDS IN POROUS MEDIA.

International Journal of Modern Physics B, v. 17, n. 01n02, p. 99-102, jan 2003. ISSN 0217-9792. Available from Internet: <http://discovery.ucl.ac.uk/29827/http: //www.worldscientific.com/doi/abs/10.1142/S021797920301714X $>$. Cited in page 20.

BORRVALL, T.; PETERSSON, J. Topology optimization of fluids in Stokes flow. International Journal for Numerical Methods in Fluids, v. 41, n. 1, p. 77-107, jan. 2003. ISSN 0271-2091. Available from Internet: <http://onlinelibrary.wiley.com/doi/ 10.1002/fld.426/abstracthttp://doi.wiley.com/10.1002/fld.426>. Cited 9 times in pages $18,19,25,26,28,30,51,52$, and 55 . 
BOYD, J.; BUICK, J. M.; GREEN, S. Analysis of the Casson and Carreau-Yasuda non-Newtonian blood models in steady and oscillatory flows using the lattice Boltzmann method. Physics of Fluids, v. 19, n. 9, p. 093103, 2007. ISSN 10706631. Available from Internet: <http://scitation.aip.org/content/aip/journal/pof2/19/9/10.1063/1.2772250>. Cited in page 14

DENG, Y.; LIU, Z.; WU, Y. Topology optimization of steady and unsteady incompressible navier-stokes flows driven by body forces. Structural and Multidisciplinary Optimization, Springer-Verlag, v. 47, n. 4, p. 555-570, 2013. ISSN 1615-147X. Available from Internet: <http://dx.doi.org/10.1007/s00158-012-0847-8>. Cited in page 26.

EVGRAFOV, A. The limits of porous materials in the topology optimization of stokes flows. Applied Mathematics and Optimization, Springer-Verlag, v. 52, n. 3, p. 263-277, 2005. ISSN 0095-4616. Available from Internet: <http: //dx.doi.org/10.1007/s00245-005-0828-z>. Cited in page 26.

EVJU, Ø. Sensitivity Analysis of Simulated Blood Flow in Cerebral Aneurysms. 2011. Cited in page 14 .

FITZGIBBON, G. M.; KAFKA, H. P.; LEACH, A. J.; KEON, W. J.; HOOPER, G.; BURTON, J. R. Coronary bypass graft fate and patient outcome: Angiographic follow-up of 5,065 grafts related to survival and reoperation in 1,388 patients during 25 years. Journal of the American College of Cardiology, v. 28, n. 3, p. 616 - 626, 1996. ISSN 0735-1097. Available from Internet: <http: //www.sciencedirect.com/science/article/pii/0735109796002069>. Cited in page 13 .

FUNKE, S. The Automation of PDE-constrained Optimisation and Its Applications. Department of Earth Science and Engineering, Imperial College London, 2013. Available from Internet: <https://books.google.com.br/books?id=ltLujgEACAAJ $>$. Cited 2 times in pages 35 and 46.

GERSBORG-HANSEN, A.; SIGMUND, O.; HABER, R. Topology optimization of channel flow problems. Structural and Multidisciplinary Optimization, Springer-Verlag, v. 30, n. 3, p. 181-192, 2005. ISSN 1615-147X. Available from Internet: <http://dx.doi.org/10.1007/s00158-004-0508-7>. Cited 2 times in pages 22 and 26.

GIJSEN, F.; VOSSE, F. van de; JANSSEN, J. The influence of the non-Newtonian properties of blood on the flow in large arteries: steady flow in a carotid bifurcation model. Journal of Biomechanics, v. 32, n. 6, p. 601-608, jun 1999. ISSN 00219290. Available from Internet: <http://linkinghub.elsevier.com/retrieve/pii/S0021929099000159>. Cited in page 13 .

HUNDERTMARK-ZAUŠKOVÁ, A.; LUKÁČOVÁ-MEDVID'OVÁ, M. Numerical study of shear-dependent non-Newtonian fluids in compliant vessels. Computers \& Mathematics with Applications, Elsevier Ltd, v. 60, n. 3, p. 572-590, aug 2010. ISSN 08981221. Available from Internet: <http://dx.doi.org/10.1016/j.camwa.2010.05.004http: //linkinghub.elsevier.com/retrieve/pii/S0898122110003445>. Cited in page 14.

HYUN, J.; WANG, S.; YANG, S. Topology optimization of the shear thinning non-newtonian fluidic systems for minimizing wall shear stress. Comput. Math. Appl., Pergamon Press, Inc., Tarrytown, NY, USA, v. 67, n. 5, p. 1154-1170, mar. 2014. ISSN 0898-1221. Available from Internet: <http://dx.doi.org/10.1016/j.camwa.2013.12.013> Cited 4 times in pages 16, 19, 27, and 29. 
KLARBRING, A.; PETERSSON, J.; TORSTEnfELT, B.; KARLSSON, M. Topology optimization of flow networks. Computer Methods in Applied Mechanics and Engineering, v. 192, n. 35-36, p. 3909-3932, aug 2003. ISSN 00457825. Available from Internet: <http://linkinghub.elsevier.com/retrieve/pii/S0045782503003931>. Cited in page 26 .

KOGA, A. A. Projeto de Dispositivos de Microcanais Utilizando o Método de Otimização Topológica. 2010. Cited 2 times in pages 15 and 26.

LOGG, A.; MARDAL, K.-A.; WELLS, G. N. et al. Automated Solution of Differential Equations by the Finite Element Method. [S.1.]: Springer, 2012. ISBN 978-3-642-23098-1. Cited 2 times in pages 23 and 45.

MACOSKO, C. Rheology: Principles, Measurements, and Applications. Wiley, 1994. ISBN 9780471185758. Available from Internet: <https://books.google.com.br/ books?id=Kai7QgAACAAJ $>$, Cited in page 20.

MUMPS Guide. MUltifrontal Massively Parallel Solver. 4. ed. MUMPS, 2011. 1-54 p. Available from Internet: <http://mumps.enseeiht.fr/http://graal.ens-lyon.fr/MUMPS/> Cited in page 45 .

MURAKAMI, Y.; MIKIC, B. Parametric optimization of multichanneled heat sinks for VLSI chip cooling. IEEE Transactions on Components and Packaging Technologies, v. 24, n. 1, p. 2-9, mar 2001. ISSN 15213331. Available from Internet: $<$ http://ieeexplore.ieee.org/lpdocs/epic03/wrapper.htm?arnumber=910795>. Cited in page 15 .

PINGEN, G.; EVGRAFOV, A.; MAUTE, K. Topology optimization of flow domains using the lattice boltzmann method. Structural and Multidisciplinary Optimization, Springer-Verlag, v. 34, n. 6, p. 507-524, 2007. ISSN 1615-147X. Available from Internet: <http://dx.doi.org/10.1007/s00158-007-0105-7>. Cited in page 26.

PINGEN, G.; MAUTE, K. Optimal design for non-newtonian flows using a topology optimization approach. Comput. Math. Appl., Pergamon Press, Inc., Tarrytown, NY, USA, v. 59, n. 7, p. 2340-2350, abr. 2010. ISSN 0898-1221. Available from Internet: <http://dx.doi.org/10.1016/j.camwa.2009.08.044>, Cited 6 times in pages 16, 19, 27, 29, 52 , and 54 .

PIRONNEAU, O. On optimum design in fluid mechanics. Journal of Fluid Mechanics, v. 64 , n. 1, p. 97-110, 1974. Cited in page 52 .

PROBST, M.; LüLFESMANN, M.; NICOLAI, M.; BüCKER, H.; BEHR, M.; BISCHOF, C. Sensitivity of optimal shapes of artificial grafts with respect to flow parameters. Computer Methods in Applied Mechanics and Engineering, v. 199, n. 17-20, p. 997 - 1005, 2010. ISSN 0045-7825. Available from Internet: $<$ http://www.sciencedirect.com/science/article/pii/S0045782509003880>. Cited in page 31 .

QUARTERONI, A.; ROZZA, G. Optimal Control and Shape Optimization of Aorto-Coronaric Bypass Anastomoses. Mathematical Models and Methods in Applied Sciences, v. 13, n. 12, p. 1801-1823, 2003. ISSN 0218-2025. Cited in page 31. 
ROBERTSON, A. M.; SEQUEIRA, A.; OWENS, R. G. Rheological models for blood. Milano: Springer Milan, 2009. 211-241 p. ISBN 978-88-470-1152-6. Cited 2 times in pages 13 and 19 .

ROBSON, J. A finite element approximation of non-Newtonian flow. Tese (Doutorado) - PhD Thesis, 2003. Cited in page 18 .

ROMERO, J.; SILVA, E. A topology optimization approach applied to laminar flow machine rotor design. Computer Methods in Applied Mechanics and Engineering, v. 279, p. 268-300, sep 2014. ISSN 00457825. Available from Internet: <www.elsevier.com/ locate/cmahttp://linkinghub.elsevier.com/retrieve/pii/S0045782514002151>. Cited 4 times in pages 21, 22, 33, and 39.

SIEBERT, M. W.; FODOR, P. S. Newtonian and Non-Newtonian Blood Flow over a Backward- Facing Step - A Case Study. Proceedings of the COMSOL Conference 2009 Boston, 2009. Cited 3 times in pages 48, 49, and 50.

SLAUGHTER, M.; PAGANI, F.; MCGEE, E.; BIRKS, E.; COTTS, W.; GREGORIC, I.; Howard Frazier, O.; ICENOGLE, T.; NAJJAR, S.; BOYCE, S.; ACKER, M.; JOHN, R.; HATHAWAY, D.; NAJARIAN, K.; AARONSON, K. Heartware ventricular assist system for bridge to transplant: Combined results of the bridge to transplant and continued access protocol trial. Journal of Heart and Lung Transplantation, Elsevier USA, v. 32, n. 7, p. 675-683, 7 2013. ISSN 1053-2498. Cited in page 13.

SOKOłOWSKI, J.; STEBEL, J. Shape optimization for non-Newtonian fluids in time-dependent domains. Evolution Equations and Control Theory, v. 3, n. 2, p. 331-348, may 2014. ISSN 2163-2480. Available from Internet: $<$ http://www.aimsciences.org/journals/displayArticlesnew.jsp?paperID=9922>. Cited in page 14.

SOUSA, L. C.; CASTRO, C. F.; ANTÓNIO, C. C. Shape optimization of an artificial bypass graft using genetic algorithms. 2nd International Conference on Engineering Optimization, n. 2, p. 6-11, 2010. Cited in page 14

SUZUKI, K.; KIKUCHI, N. A homogenization method for shape and topology optimization. Computer Methods in Applied Mechanics and Engineering, v. 93, n. 3, p. 291 - 318, 1991. ISSN 0045-7825. Available from Internet: <http://www.sciencedirect.com/science/article/pii/0045782591902452>. Cited in page 25 .

Sá, L. F. N. d. Topology Optimization Method applied to Laminar Flow Machine Rotor Design Topology Optimization Method applied to Laminar Flow Machine Rotor Design. 2016. Cited in page 25.

TAYLOR, C.; HOOD, P. A numerical solution of the Navier-Stokes equations using the finite element technique. Computers \& Fluids, v. 1, n. 1, p. 73-100, jan 1973. ISSN 00457930. Available from Internet: <http://linkinghub.elsevier.com/retrieve/pii/ 0045793073900273>. Cited in page 23 .

WÄCHTER, A.; BIEGLER, L. T. On the implementation of an interior-point filter line-search algorithm for large-scale nonlinear programming. Mathematical Programming, v. 106, n. 1, p. 25-57, mar 2006. ISSN 0025-5610. Available from 
Internet: <http://dx.doi.org/10.1007/s10107-004-0559-yhttp://link.springer.com/10.1007/ s10107-004-0559-y $>$. Cited in page 46.

WIKER, N.; KLARBRING, A.; BORRVALL, T. Topology optimization of regions of Darcy and Stokes flow. n. July 2006, p. 1374-1404, 2007. Cited 2 times in pages 26 and 28 ,

ZHANG, B.; LIU, X. Topology optimization study of arterial bypass configurations using the level set method. Structural and Multidisciplinary Optimization, v. 51, n. 3, p. 773-798, mar 2015. ISSN 1615-147X. Available from Internet:

$<$ http://link.springer.com/10.1007/s00158-014-1175-y > Cited 5 times in pages 16,27 , 57, 58, and 61.

ZHU, B.; ZHANG, X.; FATIKOW, S. A multi-objective method of hinge-free compliant mechanism optimization. Structural and Multidisciplinary Optimization, v. 49, n. 3, p. 431-440, 2014. ISSN 1615-1488. Available from Internet: <http: //dx.doi.org/10.1007/s00158-013-1003-9>. Cited 2 times in pages 32 and 41. 